\title{
Dark Matter Candidates from Particle Physics and Methods of Detection
}

\author{
Jonathan L. Feng \\ Department of Physics and Astronomy, University of California, Irvine, CA 92697, \\ USA
}

The identity of dark matter is a question of central importance in both astrophysics and particle physics. In the past, the leading particle candidates were cold and collisionless, and typically predicted missing energy signals at particle colliders. However, recent progress has greatly expanded the list of well-motivated candidates and the possible signatures of dark matter. This review begins with a brief summary of the standard model of particle physics and its outstanding problems. We then discuss several dark matter candidates motivated by these problems, including WIMPs, superWIMPs, light gravitinos, hidden dark matter, sterile neutrinos, and axions. For each of these, we critically examine the particle physics motivations and present their expected production mechanisms, basic properties, and implications for direct and indirect detection, particle colliders, and astrophysical observations. Upcoming experiments will discover or exclude many of these candidates, and progress may open up an era of unprecedented synergy between studies of the largest and smallest observable length scales.

\section{Contents}

I. INTRODUCTION 3

II. THE STANDARD MODEL OF PARTICLE PHYSICS 4

A. Particles 5

B. Problems 6

1. THE GAUGE HIERARCHY PROBLEM 6

2. THE NEW PHYSICS FLAVOR PROBLEM 7

3. THE NEUTRINO MASS PROBLEM

4. THE STRONG CP PROBLEM 8

5. OTHER PROBLEMS 8

III. WIMPS

A. Thermal Freeze Out

1. THE WIMP MIRACLE 9

2. STABILITY AND LEP'S COSMOLOGICAL LEGACY 11

3. IMPLICATIONS FOR DETECTION 12

B. Candidates 13

1. NEUTRALINOS 13

2. KALUZA-KLEIN DARK MATTER

3. OTHERS 
C. Direct Detection 16

D. Indirect Detection

E. Particle Colliders 23

IV. SUPERWIMPS 25]

A. Production Mechanisms 25

1. DECAYS [25]

2. REHEATING 25

B. Candidates 27

1. WEAK-SCALE GRAVITINOS 27

2. OTHERS 28

C. Indirect Detection 28

D. Particle Colliders

E. Astrophysical Signals

1. COSMIC MICROWAVE BACKGROUND

2. BIG BANG NUCLEOSYNTHESIS

3. SMALL SCALE STRUCTURE

V. LIGHT GRAVITINOS 32

A. Thermal Production 32

1. GAUGE-MEDIATED SUPERSYMMETRY BREAKING

2. RELIC DENSITY

B. Particle Colliders

VI. HIDDEN DARK MATTER

A. Thermal Freeze Out 35

1. CONSTRAINTS ON TEMPERATURE AND DEGREES OF FREEDOM

2. THE WIMPLESS MIRACLE

B. Candidates

C. Direct Detection

D. Indirect Detection

E. Particle Colliders

F. Astrophysical Signals 41

VII. STERILE NEUTRINOS

A. Production Mechanisms 43

B. Indirect Detection 45

C. Astrophysical Signals 45

VIII. AXIONS

A. Production Mechanisms

B. Direct Detection 48

IX. CONCLUSIONS

ACKNOWLEDGMENTS

References [50] 


\section{INTRODUCTION}

The evidence that dark matter is required to make sense of our Universe has been building for some time. In 1933 Fritz Zwicky found that the velocity dispersion of galaxies in the Coma cluster of galaxies was far too large to be supported by the luminous matter (1). In the 1970s, Vera Rubin and collaborators (2; 3) and Albert Bosma (4) measured the rotation curves of individual galaxies and also found evidence for non-luminous matter. This and other "classic" evidence for non-luminous matter (see, e.g., Ref. (5)) has now been supplemented by data from weak (6) and strong (7) lensing, hot gas in clusters (8), the Bullet Cluster (9), Big Bang nucleosynthesis (BBN) (10), further constraints from large scale structure (11), distant supernovae (12; 13), and the cosmic microwave background (CMB) (14).

Together, these data now provide overwhelming evidence for the remarkable fact that not only is there non-luminous matter in our Universe, but most of it is not composed of baryons or any of the other known particles. Current data imply that dark matter is five times more prevalent than normal matter and accounts for about a quarter of the Universe. More precisely, these data constrain the energy densities of the Universe in baryons, non-baryonic dark matter (DM), and dark energy $\Lambda$ to be (14)

$$
\begin{aligned}
\Omega_{\mathrm{B}} & \simeq 0.0456 \pm 0.0016 \\
\Omega_{\mathrm{DM}} & \simeq 0.227 \pm 0.014 \\
\Omega_{\Lambda} & \simeq 0.728 \pm 0.015
\end{aligned}
$$

Despite this progress, all of the evidence for dark matter noted above is based on its gravitational interactions. Given the universality of gravity, this evidence does little to pinpoint what dark matter is. At the same time, the identity of dark matter has far-reaching implications: in astrophysics, the properties of dark matter determine how structure forms and impact the past and future evolution of the Universe; and in particle physics, dark matter is the leading empirical evidence for new particles, and there are striking hints that it may be linked to attempts to understand electroweak symmetry breaking, the leading puzzle in the field today. The identity of dark matter is therefore of central importance in both fields and ties together studies of the Universe at both the largest and smallest observable length scales.

In this review, we discuss some of the leading dark matter candidates and their implications for experiments and observatories. The wealth of recent cosmological data does constrain some dark matter properties, such as its self-interactions and its temperature at the time of matter-radiation equality. Nevertheless, it is still not at all difficult to invent new particles that satisfy all the constraints, and there are candidates motivated by minimality, particles motivated by possible experimental anomalies, and exotic possibilities motivated primarily by the desire of clever iconoclasts to highlight how truly ignorant we are about the nature of dark matter.

Here we will focus on dark matter candidates that are motivated not only by cosmology, but also by robust problems in particle physics. For this reason, this review begins with a brief summary of the standard model of particle physics, highlighting its basic features and some of its problems. As we will see, particle physics provides strong motivation for new particles, and in many cases, these particles have just the right properties to be dark matter. We will find that many of them predict signals that are within reach of current and near future experiments. We will also find that unusual predictions for astrophysics emerge, and 


\begin{tabular}{|c|c|c|c|c|c|c|}
\hline & WIMPs & SuperWIMPs & Light $\tilde{G}$ & Hidden DM & Sterile $\nu$ & Axions \\
\hline Motivation & GHP & GHP & $\begin{array}{l}\text { GHP } \\
\text { NPFP }\end{array}$ & $\begin{array}{l}\text { GHP } \\
\text { NPFP }\end{array}$ & $\nu$ Mass & Strong CP \\
\hline $\begin{array}{l}\text { Naturally } \\
\text { Correct } \Omega\end{array}$ & Yes & Yes & No & Possible & No & No \\
\hline $\begin{array}{l}\text { Production } \\
\text { Mechanism }\end{array}$ & Freeze Out & Decay & Thermal & Various & Various & Various \\
\hline Mass Range & $\mathrm{GeV}-\mathrm{TeV}$ & $\mathrm{GeV}-\mathrm{TeV}$ & $\mathrm{eV}-\mathrm{keV}$ & $\mathrm{GeV}-\mathrm{TeV}$ & $\mathrm{keV}$ & $\mu \mathrm{eV}-\mathrm{meV}$ \\
\hline Temperature & Cold & Cold/Warm & Cold/Warm & Cold/Warm & Warm & Cold \\
\hline Collisional & & & & $\sqrt{ }$ & & \\
\hline $\begin{array}{l}\text { Early } \\
\text { Universe }\end{array}$ & & $\sqrt{ } \sqrt{ }$ & & $\sqrt{ }$ & & \\
\hline $\begin{array}{l}\text { Direct } \\
\text { Detection }\end{array}$ & $\sqrt{ } \sqrt{ }$ & & & $\sqrt{ }$ & & $\sqrt{ } \sqrt{ }$ \\
\hline $\begin{array}{l}\text { Indirect } \\
\text { Detection }\end{array}$ & $\sqrt{ } \sqrt{ }$ & $\sqrt{ }$ & & $\sqrt{ }$ & $\sqrt{ } \sqrt{ }$ & \\
\hline $\begin{array}{l}\text { Particle } \\
\text { Colliders }\end{array}$ & $\sqrt{ } \sqrt{ }$ & $\sqrt{ } \sqrt{ }$ & $\sqrt{ } \sqrt{ }$ & $\sqrt{ }$ & & \\
\hline
\end{tabular}

TABLE I Summary of dark matter particle candidates, their properties, and their potential methods of detection. The particle physics motivations are discussed in Sec. II.B. GHP and NPFP are abbreviations for the gauge hierarchy problem and new physics flavor problem, respectively. In the last five rows, $\sqrt{ } \sqrt{ }$ denotes detection signals that are generic for this class of dark matter candidate and $\sqrt{ }$ denotes signals that are possible, but not generic. "Early Universe" includes phenomena such as BBN and the CMB; "Direct Detection" implies signatures from dark matter scattering off normal matter in the laboratory; "Indirect Detection" implies signatures of late time dark matter annihilation or decay; and "Particle Colliders" implies signatures of dark matter or its progenitors produced at colliders, such as the Large Hadron Collider (LHC). See the text for details.

that cold and collisionless dark matter is far from a universal prediction, even for candidates with impeccable particle physics credentials. At the same time, it will become clear that even in favorable cases, a compelling solution to the dark matter problem will not be easy to achieve and will likely rely on synergistic progress along many lines of inquiry.

An outline of this review is provided by Table I, which summarizes the dark matter candidates discussed here, along with their basic properties and opportunities for detection. Some of the acronyms and symbols commonly used in this review are defined in Table II.

\section{THE STANDARD MODEL OF PARTICLE PHYSICS}

The standard model (SM) of particle physics is a spectacularly successful theory of elementary particles and their interactions. For a brief, pedagogical introduction, see, e.g., Ref. (15). At the same time, it has deficiencies, and the open questions raised by the SM 


\begin{tabular}{ll}
\hline \hline$\chi$ & lightest neutralino, a supersymmetric dark matter candidate \\
$\tilde{G}$ & gravitino, a supersymmetric dark matter candidate \\
$G_{N}$ & Newton's gravitational constant \\
GMSB & gauge-mediated supersymmetry breaking \\
LKP & lightest Kaluza-Klein particle \\
LSP & lightest supersymmetric particle \\
NLSP & next-to-lightest supersymmetric particle \\
$M_{\mathrm{Pl}}$ & Planck mass $\simeq 1.2 \times 10^{19} \mathrm{GeV}$ \\
$M_{*}$ & reduced Planck mass $\simeq 2.4 \times 10^{18} \mathrm{GeV}$ \\
minimal & a simple version of the MSSM specified by 5 parameters \\
supergravity & supersymmetric standard model with minimal number of extra particles \\
MSSM & standard model of particle physics \\
SM & scalar superpartner of the tau lepton \\
stau & superweakly-interacting massive particle \\
superWIMP & universal extra dimensions \\
UED & weakly-interacting massive particle \\
WIMP & general dark matter candidate \\
$X$ &
\end{tabular}

TABLE II Definitions of acronyms and symbols commonly used in this review.

motivate many of the leading dark matter candidates and provide guidance for dark matter searches. We begin here with a brief review of the SM's basic features and open problems, focusing on those that are most relevant for dark matter.

\section{A. Particles}

The particles of the SM are shown in Fig. 1. They may be divided into three categories:

- Spin 1/2 Fermions. These matter particles include six flavors of quarks (up, down, charm, strange, bottom, and top), three flavors of charged leptons (electrons, muons, and taus), and three flavors of neutral leptons (the electron, muon, and tau neutrinos). These are grouped into three generations, as indicated in Fig. 1.

- Spin 1 Gauge Bosons. These force carrying particles include the photon $\gamma$, which mediates electromagnetism; eight gluons $g$, which mediate the strong force; and the $W$ and $Z$ gauge bosons, which mediate the weak interactions. The photon and gluons are massless, but the $W$ and $Z$ have masses $80 \mathrm{GeV}$ and $91 \mathrm{GeV}$, respectively.

- Spin 0 Higgs Boson. The SM Higgs particle is a spin 0 boson. Although the Higgs boson has not yet been discovered, its mass is constrained by a variety of collider results. Assuming the SM, null results from direct searches at the LEP $e^{+} e^{-}$collider require $m_{h}>114.4 \mathrm{GeV}(16)$. Given this constraint, precision measurements of electroweak observables at LEP require $m_{h}<186 \mathrm{GeV}$ (17). In addition, the Tevatron $p \bar{p}$ collider, currently running, excludes the region $162 \mathrm{GeV}<m_{h}<166 \mathrm{GeV}$ (18). These bounds may be relaxed in extensions of the SM, but even in such theories, very general arguments require $m_{h} \lesssim 1 \mathrm{TeV}$ (see, e.g., Ref. (19)). 


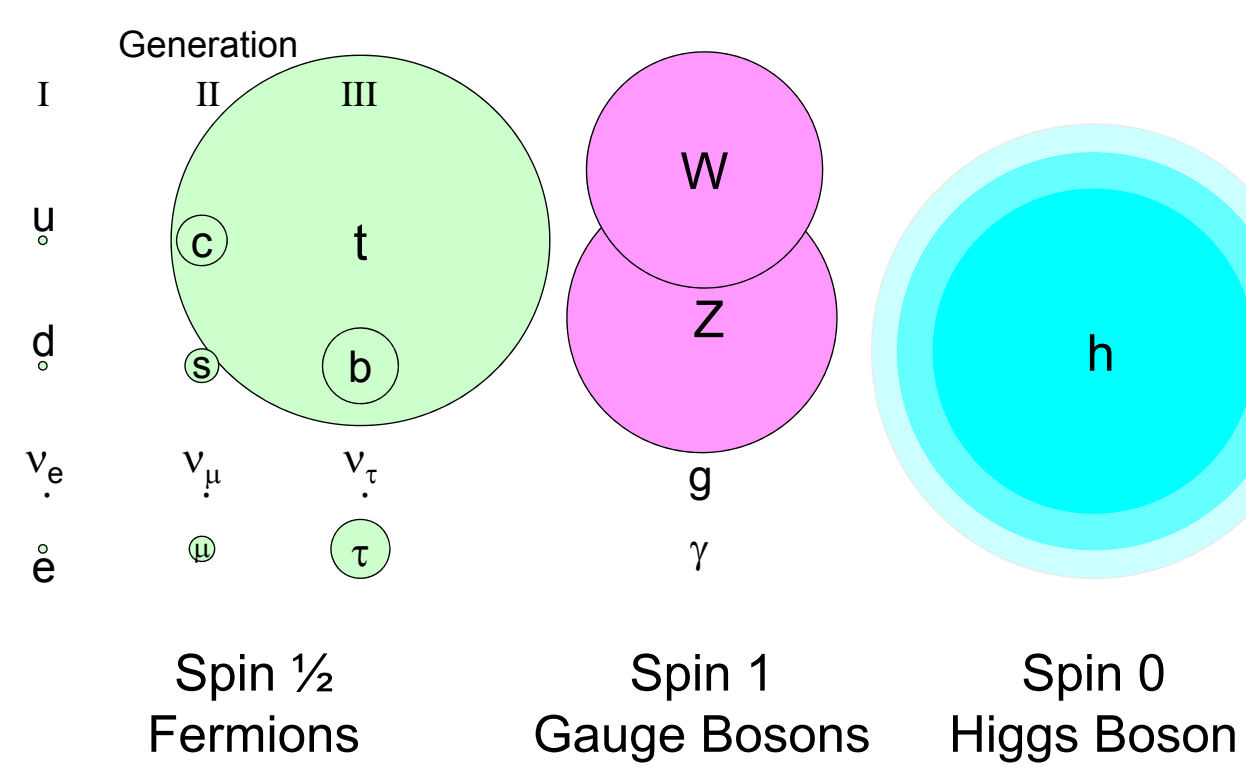

FIG. 1 The particles of the standard model, represented by circles whose areas are proportional to their masses. The photon and gluon are massless. The Higgs boson has not yet been discovered — its mass has been taken in the allowed range $114.4 \mathrm{GeV}<m_{h}<186 \mathrm{GeV}$ (see text).

None of these SM particles is a good dark matter candidate. Most of the matter particles are unstable, with lifetimes far shorter than the age of the Universe. The remaining particles are the six lightest: the electron, the up and down quarks, which may form stable protons and neutrons in nuclei, and the three neutrinos. Electrons may contribute significantly to dark matter only if they are neutralized through binding with protons, but protons (and neutrons) contribute to the baryonic energy density $\Omega_{B}$, which is too small to be all of dark matter. In addition, current upper bounds on neutrino masses from particle physics and cosmology imply that the neutrino relic density $\Omega_{\nu} \simeq \sum_{i} m_{\nu_{i}} / 47 \mathrm{eV} \lesssim 0.012$ (14). The evidence for dark matter therefore requires particles beyond the SM.

\section{B. Problems}

In addition to the need for dark matter, other problems motivate physics beyond the SM. These problems are of two types. The first and most severe are experimental data that the SM cannot explain; at present, aside from the existence of dark matter, these are confined to the neutrino sector and are described in Sec. II.B.3. The second are experimental data that can be explained, but only for seemingly unnatural choices of parameters. All of the remaining problems are of this type.

\section{THE GAUGE HIERARCHY PROBLEM}

The gauge hierarchy problem is the question of why the physical Higgs boson mass $m_{h}$ is so small. What is the natural value for $m_{h}$ ? We know of three fundamental constants: the speed of light $c$, Planck's constant $h$, and Newton's gravitational constant $G_{N}$. One combination of these has dimensions of mass, the Planck mass $M_{\mathrm{Pl}} \equiv \sqrt{h c / G_{N}} \simeq 1.2 \times 10^{19} \mathrm{GeV}$. We therefore expect dimensionful parameters to be either 0 , if enforced by a symmetry, or of 
the order of $M_{\mathrm{Pl}}$. In the SM, electroweak symmetry is broken, and the Higgs boson mass is non-zero. The gauge hierarchy problem is the question of why $m_{h} \sim 100 \mathrm{GeV} \ll M_{\mathrm{Pl}}$.

This problem is exacerbated in the SM by quantum corrections. The physical mass of the SM Higgs boson is $m_{h}^{2}=m_{h 0}^{2}+\Delta m_{h}^{2}$, where $m_{h 0}^{2}$ is the tree-level mass, and

$$
\Delta m_{h}^{2} \sim \frac{\lambda^{2}}{16 \pi^{2}} \int^{\Lambda} \frac{d^{4} p}{p^{2}} \sim \frac{\lambda^{2}}{16 \pi^{2}} \Lambda^{2}
$$

is the quantum correction resulting from loop-level diagrams, where the integral is over the momenta of particles in the loops. The parameter $\lambda$ is an $\mathcal{O}(1)$ dimensionless coupling, and $\Lambda$ is the energy scale at which the SM is no longer a valid description of nature. Because $\Delta m_{h}^{2}$ is proportional to $\Lambda^{2}$, it is natural to expect the Higgs mass to be pulled up to within an order of magnitude of $\Lambda$ by quantum corrections. In the SM with $\Lambda \sim M_{\mathrm{Pl}}$, this implies that $m_{h 0}^{2}$ and $\Delta m_{h}^{2}$ must cancel to 1 part in $10^{36}$ to yield the correct physical Higgs mass, which is hardly reasonable.

The gauge hierarchy problem may be eliminated if $\Lambda \lesssim 1 \mathrm{TeV}$, implying new physics at the weak scale $m_{\text {weak }} \sim 10 \mathrm{GeV}-\mathrm{TeV}$. Alternatively, the Higgs boson may not be a fundamental scalar, but in this case, too, its structure requires new physics at the weak scale (20). For these reasons, every attempt to ameliorate the gauge hierarchy problem so far has implied new particles with mass around $m_{\text {weak }}$. The gauge hierarchy problem is the leading motivation for dark matter candidates, such as WIMPs and superWIMPs, weaklyand superweakly-interacting massive particles, that are the topics of Secs. III and IV below.

\section{THE NEW PHYSICS FLAVOR PROBLEM}

The gauge hierarchy problem implies new particles with mass around $m_{\text {weak }}$. Such particles typically create many new problems, however, because they may violate baryon number, lepton number, flavor, or $\mathrm{CP}$, where $\mathrm{C}$ and $\mathrm{P}$ are the discrete transformations of charge conjugation and parity, respectively. These symmetries are either beautifully preserved or violated only slightly in the SM, but there is no guarantee that new $m_{\text {weak }}$ particles will preserve them. This set of problems is collectively known as the new physics flavor problem.

The new physics flavor problem implies that not all solutions to the gauge hierarchy problem are equally elegant. For example, among supersymmetric theories, it implies that those that naturally predict very heavy squarks and sleptons, or those that predict squarks and sleptons that are highly degenerate across different generations, are favored, because these naturally suppress flavor-changing neutral currents below current constraints. This problem has implications for the direct detection of WIMPs, as discussed in Sec. III.C, and motivates light gravitino dark matter, reviewed in Sec. V] and some of the hidden sector dark matter models described in Sec. VI.

\section{THE NEUTRINO MASS PROBLEM}

Fermion masses are described in quantum field theories by terms that couple left- and right-handed fields together. In the SM, however, there are no right-handed neutrino fields, and so the SM predicts that all neutrinos are massless. The observation of neutrino flavor oscillations (21; 22), however, implies that the three neutrinos are non-degenerate, and so at least two are massive. Neutrino masses and mixing provide the most direct and compelling 
evidence that the SM of particle physics is incomplete, and this problem motivates sterile neutrino dark matter, discussed in Sec. VII.

\section{THE STRONG CP PROBLEM}

The SM Lagrangian includes the term $g_{3}^{2} \theta_{3} /\left(32 \pi^{2}\right) \epsilon^{\mu \nu \rho \sigma} G_{\mu \nu}^{\alpha} G_{\rho \sigma}^{\alpha}$, where $g_{3}$ is the coupling of the strong interactions, $\theta_{3}$ is an angle parameter, $\epsilon^{\mu \nu \rho \sigma}$ is the totally anti-symmetric 4index tensor, and $G_{\mu \nu}$ is the gluon field strength. This term contributes to CP-violating, flavor-conserving observables, such as the electric dipole moment (EDM) of the neutron $d_{e}$. For $\theta_{3} \sim 1$, one expects $d_{e} \sim 10^{-16} e \mathrm{~cm}$. The neutron EDM has not yet been observed, but current constraints already imply $d_{e}<2.9 \times 10^{-26} e \mathrm{~cm}(23)$. This is therefore a fine-tuning problem of 1 part in $10^{10}$, and it motivates axions as dark matter candidates, to be discussed in Sec. VIII.

\section{OTHER PROBLEMS}

In addition to the outstanding problems discussed above, there are other open questions raised by the SM. The SM flavor problem, distinct from the new physics flavor problem, is the puzzle of why the fermion masses have such different values, as evident in Fig. 1. The grand unification problem is the problem of trying to understand the strong, weak, and electromagnetic interactions as different manifestations of a single underlying force. In addition, at any given time there are always experimental anomalies, measurements that do not agree with SM predictions. At present, the most compelling and persistent discrepancy is in the anomalous magnetic moment of the muon, which disagrees with the SM prediction at the level of $3.4 \sigma(24)$. Although the problems described in this paragraph do not typically motivate dark matter candidates on their own, they do sometimes play a supporting role, as discussed below.

Finally, note that $m_{h 0}$ is only one of two dimensionful parameters in the SM: there is also the term $\bar{\Lambda}^{4}$, which contributes to dark energy or the cosmological constant. Equation (3) implies that the total energy density in dark energy is $\Lambda \simeq(2.76 \mathrm{meV})^{4}$. If the natural value of $\bar{\Lambda}$ is $M_{\mathrm{Pl}}^{4}$, it must cancel other contributions to 1 part in $10^{122}$, a fine-tuning that dwarfs even the gauge hierarchy problem. This is the cosmological constant problem. Although one might hope for a unified solution to the cosmological constant and dark matter problems, at present there is little indication that they are related, and we will assume they are decoupled in this review.

\section{WIMPS}

WIMPs have mass in the range $m_{\text {weak }} \sim 10 \mathrm{GeV}-\mathrm{TeV}$ and tree-level interactions with the $W$ and $Z$ gauge bosons, but not with gluons or photons. WIMPs are the most studied dark matter candidates, as they are found in many particle physics theories, naturally have the correct relic density, and may be detected in many ways. In this section, we discuss their production through thermal freeze out, the examples of neutralino and Kaluza-Klein dark matter, and their implications for direct detection, indirect detection, and particle colliders. 


\section{A. Thermal Freeze Out}

\section{THE WIMP MIRACLE}

If a WIMP exists and is stable, it is naturally produced with a relic density consistent with that required of dark matter. This tantalizing fact, sometimes referred to as the "WIMP miracle," implies that particles that are motivated by the gauge hierarchy problem, a purely microphysical puzzle, are excellent dark matter candidates.

Dark matter may be produced in a simple and predictive manner as a thermal relic of the Big Bang (25; 26, 27; 28). The evolution of a thermal relic's number density is shown in Fig. 2. Initially the early Universe is dense and hot, and all particles are in thermal equilibrium. The Universe then cools to temperatures $T$ below the dark matter particle's mass $m_{X}$, and the number of dark matter particles becomes Boltzmann suppressed, dropping exponentially as $e^{-m_{X} / T}$. The number of dark matter particles would drop to zero, except that, in addition to cooling, the Universe is also expanding. In stage (3), the Universe becomes so large and the gas of dark matter particles becomes so dilute that they cannot find each other to annihilate. The dark matter particles then "freeze out," with their number asymptotically approaching a constant, their thermal relic density. Note that freeze out, also known as chemical decoupling, is distinct from kinetic decoupling; after thermal freeze out, interactions that change the number of dark matter particles become negligible, but interactions that mediate energy exchange between dark matter and other particles may remain efficient.

This process is described quantitatively by the Boltzmann equation

$$
\frac{d n}{d t}=-3 H n-\left\langle\sigma_{A} v\right\rangle\left(n^{2}-n_{\mathrm{eq}}^{2}\right)
$$

where $n$ is the number density of the dark matter particle $X, H$ is the Hubble parameter, $\left\langle\sigma_{A} v\right\rangle$ is the thermally averaged annihilation cross section, and $n_{\text {eq }}$ is the dark matter number density in thermal equilibrium. On the right-hand side of Eq. (5), the first term accounts for dilution from expansion. The $n^{2}$ term arises from processes $X X \rightarrow$ SM SM that destroy $X$ particles, where SM denotes SM particles, and the $n_{\mathrm{eq}}^{2}$ term arises from the reverse process $\mathrm{SM} \mathrm{SM} \rightarrow X X$, which creates $X$ particles.

The thermal relic density is determined by solving the Boltzmann equation numerically. A rough analysis is highly instructive, however. Defining freeze out to be the time when $n\left\langle\sigma_{A} v\right\rangle=H$, we have

$$
n_{f} \sim\left(m_{X} T_{f}\right)^{3 / 2} e^{-m_{X} / T_{f}} \sim \frac{T_{f}^{2}}{M_{\mathrm{Pl}}\left\langle\sigma_{A} v\right\rangle},
$$

where the subscripts $f$ denote quantities at freeze out. The ratio $x_{f} \equiv m_{X} / T_{f}$ appears in the exponential. It is, therefore, highly insensitive to the dark matter's properties and may be considered a constant; a typical value is $x_{f} \sim 20$. The thermal relic density is, then,

$$
\Omega_{X}=\frac{m_{X} n_{0}}{\rho_{c}}=\frac{m_{X} T_{0}^{3}}{\rho_{c}} \frac{n_{0}}{T_{0}^{3}} \sim \frac{m_{X} T_{0}^{3}}{\rho_{c}} \frac{n_{f}}{T_{f}^{3}} \sim \frac{x_{f} T_{0}^{3}}{\rho_{c} M_{\mathrm{Pl}}}\left\langle\sigma_{A} v\right\rangle^{-1},
$$

where $\rho_{c}$ is the critical density and the subscripts 0 denote present day quantities. We see that the thermal relic density is insensitive to the dark matter mass $m_{X}$ and inversely proportional to the annihilation cross section $\left\langle\sigma_{A} v\right\rangle$. 


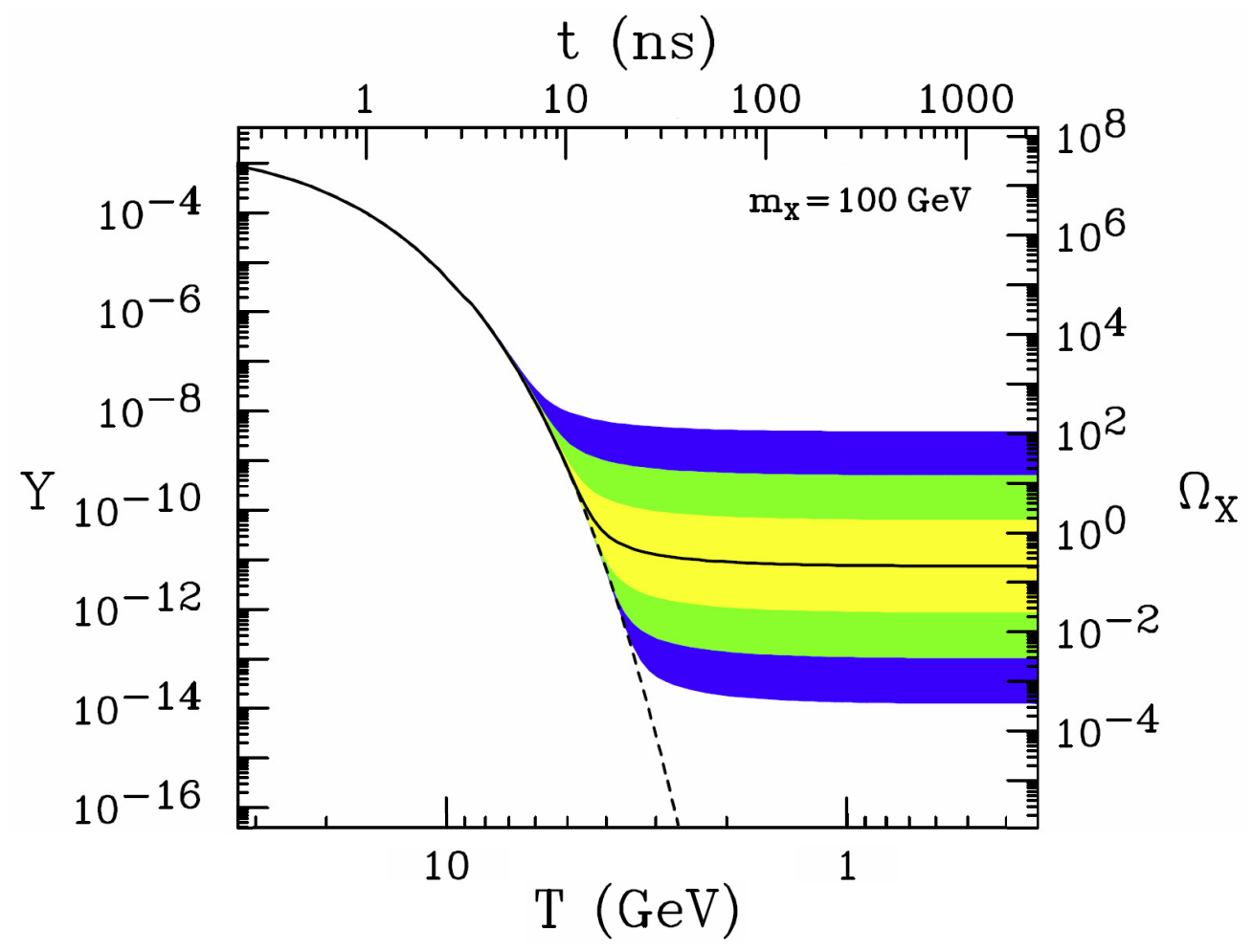

FIG. 2 The comoving number density $Y$ (left) and resulting thermal relic density (right) of a 100 $\mathrm{GeV}, P$-wave annihilating dark matter particle as a function of temperature $T$ (bottom) and time $t$ (top). The solid contour is for an annihilation cross section that yields the correct relic density, and the shaded regions are for cross sections that differ by $10,10^{2}$, and $10^{3}$ from this value. The dashed contour is the number density of a particle that remains in thermal equilibrium.

Although $m_{X}$ does not enter $\Omega_{X}$ directly, in many theories it is the only mass scale that determines the annihilation cross section. On dimensional grounds, then, the cross section can be written

$$
\sigma_{A} v=k \frac{g_{\mathrm{weak}}^{4}}{16 \pi^{2} m_{X}^{2}}\left(1 \text { or } v^{2}\right),
$$

where the factor $v^{2}$ is absent or present for $S$ - or $P$-wave annihilation, respectively, and terms higher-order in $v$ have been neglected. The constant $g_{\text {weak }} \simeq 0.65$ is the weak interaction gauge coupling, and $k$ parameterizes deviations from this estimate.

With this parametrization, given a choice of $k$, the relic density is determined as a function of $m_{X}$. The results are shown in Fig. 3. The width of the band comes from considering both $S$ - and $P$-wave annihilation, and from letting $k$ vary from $\frac{1}{2}$ to 2 . We see that a particle that makes up all of dark matter is predicted to have mass in the range $m_{X} \sim 100 \mathrm{GeV}-1 \mathrm{TeV}$; a particle that makes up $10 \%$ of dark matter has mass $m_{X} \sim 30 \mathrm{GeV}-300 \mathrm{GeV}$. This is the WIMP miracle: weak-scale particles make excellent dark matter candidates. We have neglected many details here, and there are models for which $k$ lies outside our illustrative range, sometimes by as much as an order of magnitude or two. Nevertheless, the WIMP miracle implies that many models of particle physics easily provide viable dark matter candidates, and it is at present the strongest reason to expect that central problems in particle physics and astrophysics may in fact be related. Note also that, for those who find the aesthetic nature of the gauge hierarchy problem distasteful, the WIMP miracle 


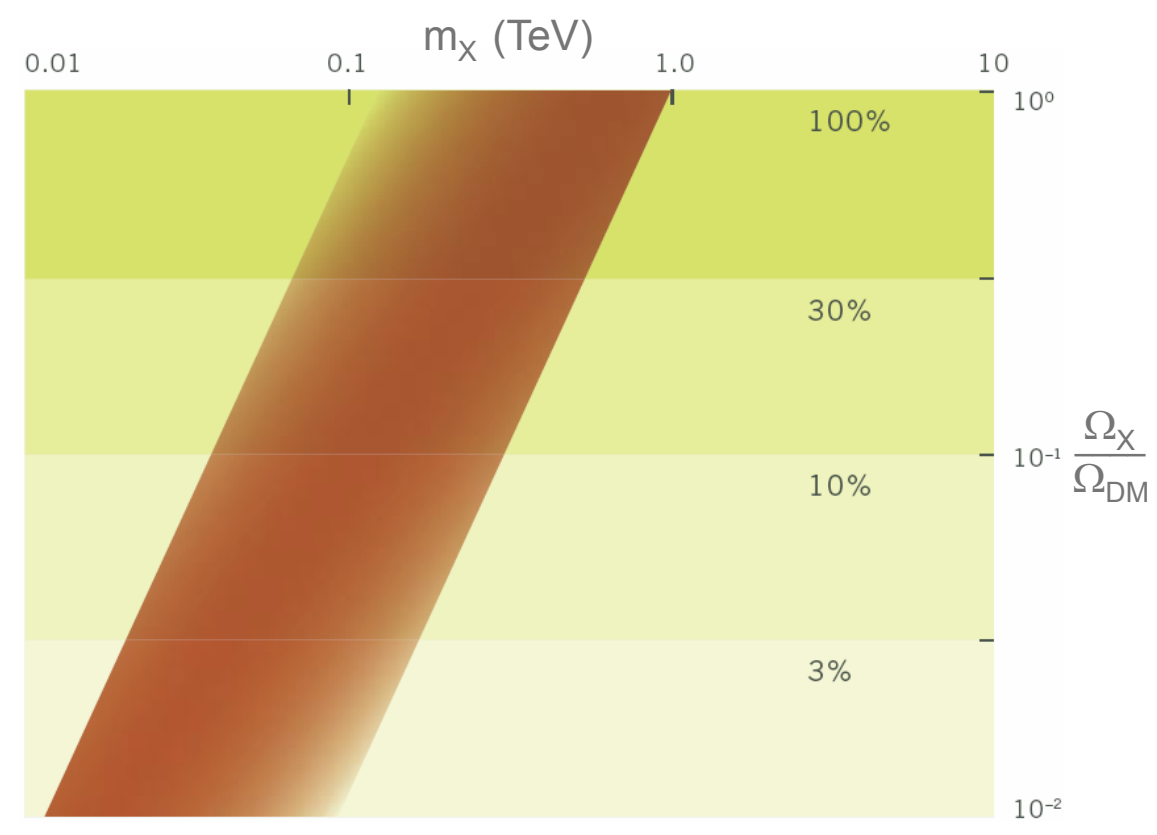

FIG. $3 \mathrm{~A}$ band of natural values in the $\left(m_{X}, \Omega_{X} / \Omega_{\mathrm{DM}}\right)$ plane for a thermal relic $X$, where $\Omega_{\mathrm{DM}} \simeq$ 0.23 is the required total dark matter density (29).

independently provides a strong motivation for new particles at the weak scale.

\section{STABILITY AND LEP'S COSMOLOGICAL LEGACY}

The entire discussion of Sec. III.A.1 assumes that the WIMP is stable. This might appear to be an unreasonable expectation; after all, all particles heavier than a GeV in the SM decay on time scales far shorter than the age of the Universe.

In fact, however, there are already indications that if new particles exist at the weak scale, at least one of them should be stable. This is the cosmological legacy of LEP, the Large Electron-Positron Collider that ran from 1989-2000. Generically, new particles introduced to solve the gauge hierarchy problem would be expected to induce new interactions

$$
\mathrm{SM} \mathrm{SM} \rightarrow \mathrm{NP} \rightarrow \mathrm{SM} \mathrm{SM}
$$

where SM and NP denote standard model and new particles, respectively. If the new particles are heavy, they cannot be produced directly, but their effects may nevertheless be seen as perturbations on the properties of SM particles. LEP, along with the Stanford Linear Collider, looked for the effects of these interactions and found none, constraining the mass scale of new particles to be above $\sim 1-10 \mathrm{TeV}$, depending on the SM particles involved (see, e.g., Ref. (30) ). At the same time, to solve the gauge hierarchy problem, the new particles cannot be decoupled completely. At the very least, the new particles should modify the quantum corrections to the Higgs boson mass given in Eq. (4). This implies that they must interact with the Higgs boson through couplings

$$
h \leftrightarrow \mathrm{NP} \mathrm{NP},
$$

and their masses should not be significantly higher than $m_{\text {weak }} \sim 10 \mathrm{GeV}-\mathrm{TeV}$. 
These apparently conflicting demands may be reconciled if there is a conserved discrete parity that requires all interactions to involve an even number of new particles (31; 32). Such a conservation law would eliminate the problematic reactions of Eq. (9), while preserving the desired interactions of Eq. (10). As a side effect, the existence of a discrete parity implies that the lightest new particle cannot decay. The lightest new particle is therefore stable, as required for dark matter. Note that pair annihilation of dark matter particles is still allowed. The prototypical discrete parity is $R$-parity, proposed for supersymmetry long before the existence of LEP bounds (33). However, the existence of LEP constraints implies that any new theory of the weak scale must confront this difficulty. The required discrete parity may be realized in many ways, depending on the new physics at the weak scale; an example in extra dimensions is given in Sec. III.B.2.

\section{IMPLICATIONS FOR DETECTION}

The WIMP miracle not only provides a model-independent motivation for dark matter at the weak scale, but it also has strong implications for how dark matter might be detected. For WIMPs $X$ to have the observed relic density, they must annihilate to other particles. Assuming that these other particles are SM particles, the necessity of $X X \rightarrow \mathrm{SM}$ SM interactions suggests three promising strategies for dark matter detection:

- Indirect detection: if dark matter annihilated in the early Universe, it must also annihilate now through $X X \rightarrow$ SM SM, and the annihilation products may be detected.

- Direct detection: dark matter can scatter off normal matter through $X \mathrm{SM} \rightarrow X \mathrm{SM}$ interactions, depositing energy that may be observed in sensitive, low background detectors.

- Particle colliders: dark matter may be produced at particle colliders through SM SM $\rightarrow$ $X X$. Such events are undetectable, but are typically accompanied by related production mechanisms, such as SM SM $\rightarrow X X+\{\mathrm{SM}\}$, where " $\{\mathrm{SM}\}$ " denotes one or more standard model particles. These events are observable and provide signatures of dark matter at colliders.

It is important to note that the WIMP miracle not only implies that such dark matter interactions must exist, it also implies that the dark matter-SM interactions must be efficient; although WIMPs may not be a significant amount of the dark matter, they certainly cannot have an energy density more than $\Omega_{\mathrm{DM}}$. Cosmology therefore provides lower bounds on interaction rates. This fact provides highly motivated targets for a diverse array of experimental searches that may be able to detect WIMPs and constrain their properties.

To summarize, viable particle physics theories designed to address the gauge hierarchy problem naturally (1) predict new particles with mass $\sim m_{\text {weak }}$ that (2) are stable and (3) have the thermal relic densities required to be dark matter. The convergence of particle physics and cosmological requirements for new states of matter has motivated many new proposals for dark matter. In the following section, we discuss some prominent examples. 


\section{B. Candidates}

\section{NEUTRALINOS}

The gauge hierarchy problem is most elegantly solved by supersymmetry. In supersymmetric extensions of the SM, every SM particle has a new, as-yet-undiscovered partner particle, which has the same quantum numbers and gauge interactions, but differs in spin by $1 / 2$. The introduction of new particles with opposite spin-statistics from the known ones supplements the SM quantum corrections to the Higgs boson mass with opposite sign contributions, modifying Eq. (4) to

$$
\left.\Delta m_{h}^{2} \sim \frac{\lambda^{2}}{16 \pi^{2}} \int^{\Lambda} \frac{d^{4} p}{p^{2}}\right|_{\mathrm{SM}}-\left.\frac{\lambda^{2}}{16 \pi^{2}} \int^{\Lambda} \frac{d^{4} p}{p^{2}}\right|_{\mathrm{SUSY}} \sim \frac{\lambda^{2}}{16 \pi^{2}}\left(m_{\mathrm{SUSY}}^{2}-m_{\mathrm{SM}}^{2}\right) \ln \frac{\Lambda}{m_{\mathrm{SUSY}}},
$$

where $m_{\mathrm{SM}}$ and $m_{\mathrm{SUSY}}$ are the masses of the SM particles and their superpartners. For $m_{\mathrm{SUSY}} \sim m_{\text {weak }}$, this is at most an $\mathcal{O}(1)$ correction, even for $\Lambda \sim M_{\mathrm{Pl}}$. This by itself stabilizes, but does not solve, the gauge hierarchy problem; one must also understand why $m_{\mathrm{SUSY}} \sim m_{\text {weak }} \ll M_{\mathrm{Pl}}$. There are, however, a number of ways to generate such a hierarchy; for a review, see Ref. (34). Given such a mechanism, the relation of Eq. (11) implies that quantum effects will not destroy the hierarchy, and the gauge hierarchy problem may be considered truly solved.

Not surprisingly, the doubling of the SM particle spectrum has many implications for cosmology. For dark matter, it is natural to begin by listing all the new particles that are electrically neutral. For technical reasons, supersymmetric models require two Higgs bosons. The neutral supersymmetric particles are then

$$
\begin{aligned}
\text { Spin } 3 / 2 \text { Fermion: } & \text { Gravitino } \tilde{G} \\
\text { Spin } 1 / 2 \text { Fermions: } & \tilde{B}, \tilde{W}, \tilde{H}_{u}, \tilde{H}_{d} \rightarrow \text { Neutralinos } \chi_{1}, \chi_{2}, \chi_{3}, \chi_{4} \\
\text { Spin 0 Scalars: } & \text { Sneutrinos } \tilde{\nu}_{e}, \tilde{\nu}_{\mu}, \tilde{\nu}_{\tau} .
\end{aligned}
$$

As indicated, the neutral spin $1 / 2$ fermions mix to form four mass eigenstates, the neutralinos. The lightest of these, $\chi \equiv \chi_{1}$, is a WIMP dark matter candidate (35; 36). The sneutrinos are not good dark matter candidates, as both their their annihilation and scattering cross sections are large, and so they are under-abundant or excluded by null results from direct detection experiments for all masses near $m_{\text {weak }}(37 ; 38)$. The gravitino is not a WIMP, but it is a viable and fascinating dark matter candidate, as discussed in Secs. IV and $\mathrm{VI}$.

A general supersymmetric extension of the SM contains many unknown parameters. To make progress, it is typical to consider specific models in which simplifying assumptions unify many parameters, and then study to what extent the conclusions may be generalized. The canonical model for supersymmetric studies is minimal supergravity, which is minimal in the sense that it includes the minimum number of particles and includes a large number of assumptions that drastically reduces the number of independent model parameters. Minimal supergravity is defined by five parameters:

$$
m_{0}, M_{1 / 2}, A_{0}, \tan \beta, \operatorname{sign}(\mu) .
$$

The most important parameters are the universal scalar mass $m_{0}$ and the universal gaugino mass $M_{1 / 2}$, both defined at the scale of grand unified theories $m_{\mathrm{GUT}} \simeq 2 \times 10^{16} \mathrm{GeV}$. 


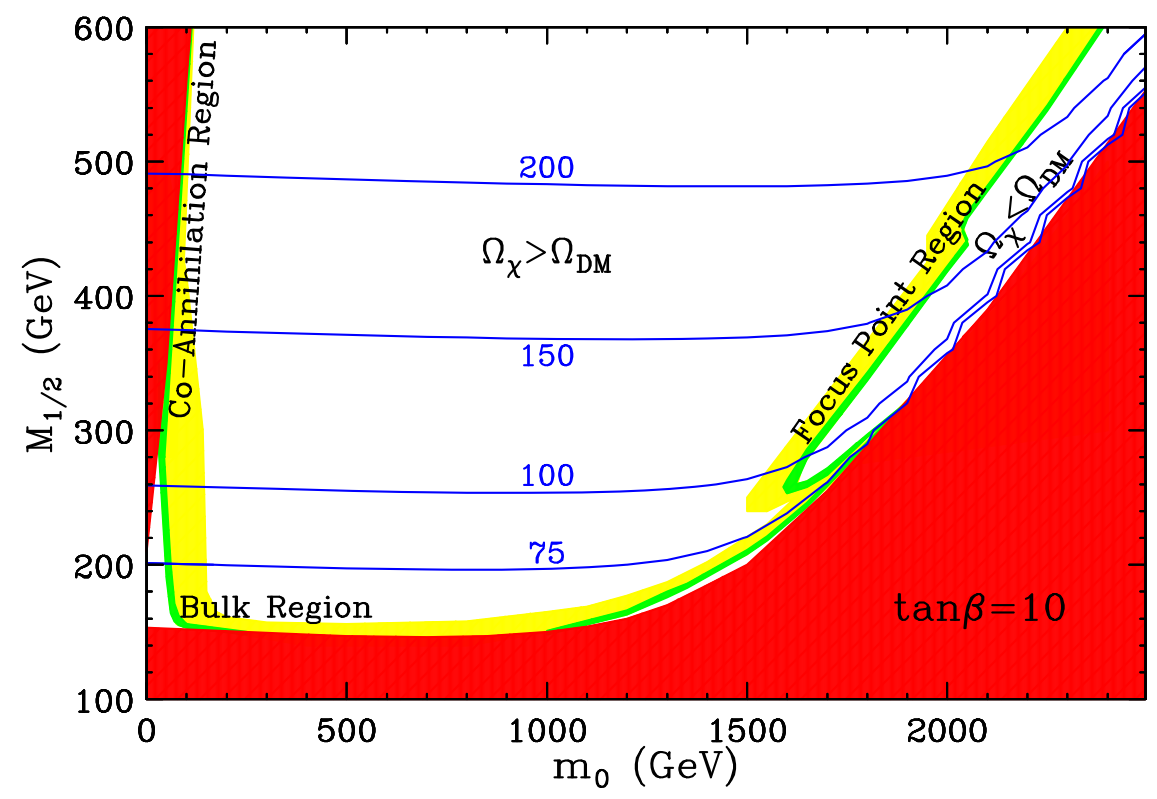

FIG. 4 Regions of minimal supergravity $\left(m_{0}, M_{1 / 2}\right)$ parameter space for fixed $A_{0}=0, \tan \beta=10$, and $\mu>0$. The green (yellow) region is cosmologically favored with $0.20<\Omega_{\chi}<0.28\left(0.2<\Omega_{\chi}<\right.$ 0.6). The names of cosmologically-favored regions (focus point, bulk, and co-annihilation) are indicated, along with regions with too much and too little dark matter. The lower right red shaded region is excluded by collider bounds on chargino masses; the upper left red region is excluded by the presence of a stable charged particle. Contours are for neutralino dark matter mass $m_{\chi}$ in GeV. Adapted from Ref. (40).

The assumption of a universal gaugino mass and the choice of $m_{\mathrm{GUT}}$ are supported by the fact that the three SM gauge couplings unify at $m_{\mathrm{GUT}}$ in supersymmetric theories (39). The assumption of scalar mass unification is much more ad hoc, but it does imply highly degenerate squarks and sleptons, which typically satisfies the constraints of the new physics flavor problem. Finally, the parameter $A_{0}$ governs the strength of cubic scalar particle interactions, and $\tan \beta$ and $\operatorname{sign}(\mu)$ are parameters that enter the Higgs boson potential. For all but their most extreme values, these last three parameters have much less impact on collider and dark matter phenomenology than $m_{0}$ and $M_{1 / 2}$.

In the context of minimal supergravity, the thermal relic density is given in the $\left(m_{0}, M_{1 / 2}\right)$ plane for fixed values of $A_{0}, \tan \beta$, and $\operatorname{sign}(\mu)$ in Fig. 4. We see that current constraints on $\Omega_{\mathrm{DM}}$ are highly constraining, essentially reducing the cosmologically favored parameter space by one dimension. The region of parameter space with the correct neutralino relic density is further divided into three regions with distinct properties: the bulk region, the focus point region, and the co-annihilation region. Of course, if one considers the full minimal supergravity parameter space, other points in the $\left(m_{0}, M_{1 / 2}\right)$ plane are possible (see, e.g., Ref. (41)); notably, at larger $\tan \beta$ there is another favored region, known as the funnel region.

Note that for much of the region with $m_{0}, M_{1 / 2} \lesssim \mathrm{TeV}, \Omega_{\chi}$ is too large. This is because neutralinos, although widely studied, are very special: they are Majorana fermions, that is, they are their own anti-particles. If the initial state neutralinos are in an $S$-wave state, the Pauli exclusion principle implies that the initial state has total spin $S=0$ and total angular momentum $J=0$. Annihilation to fermion pairs with total spin $S=1$, such as $e_{R}^{-} e_{R}^{+}$, 
is therefore $P$-wave suppressed, with an extra factor of $v^{2} \sim 0.1$ in the annihilation cross section. As a result, $\Omega_{\chi}$ is typically too large, and the correct $\Omega_{\chi}$ is achieved for relatively light neutralinos, as evident in Fig. 4 .

\section{KALUZA-KLEIN DARK MATTER}

An alternative possibility for new weak-scale physics is extra dimensions. The idea that there may be extra spatial dimensions is an old one, going back at least as far as the work of Kaluza and Klein in the 1920's (42). Their original idea is untenable, but it has many modern descendants, of which the closest living relative is universal extra dimensions (UED) (43).

In UED, all particles propagate in flat, compact extra dimensions of size $10^{-18} \mathrm{~m}$ or smaller. In the simplest UED model, minimal UED, there is one extra dimension of size $R$ compactified on a circle, with points with $y$ and $-y$ identified, where $y$ is the coordinate of the extra dimension. Every SM particle has an infinite number of partner particles, with one at every Kaluza-Klein (KK) level $n$ with mass $\sim n R^{-1}$. In contrast to supersymmetry, these partner particles have the same spin. As a result, UED models do not solve the gauge hierarchy problem; in fact, their couplings become large and non-perturbative at energies far below the Planck scale. The motivation to consider UED models is that they provide an interesting and qualitatively different alternative to supersymmetry, but it assumes that UED are a low-energy approximation to a more complete theory that resolves the gauge hierarchy problem and is well-defined up to the Planck scale.

Minimal UED parameter space is extremely simple, as it is completely determined by only two parameters: $m_{h}$, the mass of the SM Higgs boson, and $R$, the compactification radius. For the Higgs boson mass, the direct search constraints on the SM also apply in UED and require $m_{h}>114.4 \mathrm{GeV}(16)$. However, the indirect bounds are significantly weakened by the existence of many levels of KK particles, and require only $m_{h}<900 \mathrm{GeV}(300 \mathrm{GeV})$ for $R^{-1}=250 \mathrm{GeV}(1 \mathrm{TeV})$ at $90 \% \mathrm{CL}(44)$.

The simplest UED models preserve a discrete parity known as KK-parity, which implies that the lightest KK particle (LKP) is stable and a possible dark matter candidate (45; 46). The lightest KK particle (LKP) is typically $B^{1}$, the level 1 partner of the hypercharge gauge boson. The regions of parameter space with the correct $B^{1}$ thermal relic density have been investigated in a series of increasingly sophisticated studies (45; 47; 48; 49); the end results are shown in Fig. 5. As can be seen, the required LKP mass is $600 \mathrm{GeV} \lesssim m_{B^{1}} \lesssim 1.4 \mathrm{TeV}$, a heavier range than for neutralinos. This is because LKPs annihilation is through $S$-wave processes, and so is not $P$-wave suppressed, in contrast to neutralinos. Nevertheless, the required dark matter mass is still $\sim 1 \mathrm{TeV}$, as expected given the WIMP miracle.

\section{OTHERS}

Neutralinos are the prototypical WIMP, and KK dark matter provides an instructive example of WIMPs that differ in important aspects from neutralinos. There are many other examples, however. In the recent years leading up to the start of the LHC, there has been a proliferation of electroweak theories and an accompanying proliferation of WIMP candidates. These include branons in theories with large extra dimensions (50; 51), T-odd particles in little Higgs theories (32, 52), and excited states in theories with warped extra dimensions (53). As with all WIMPs, these are astrophysically equivalent, in that they are 


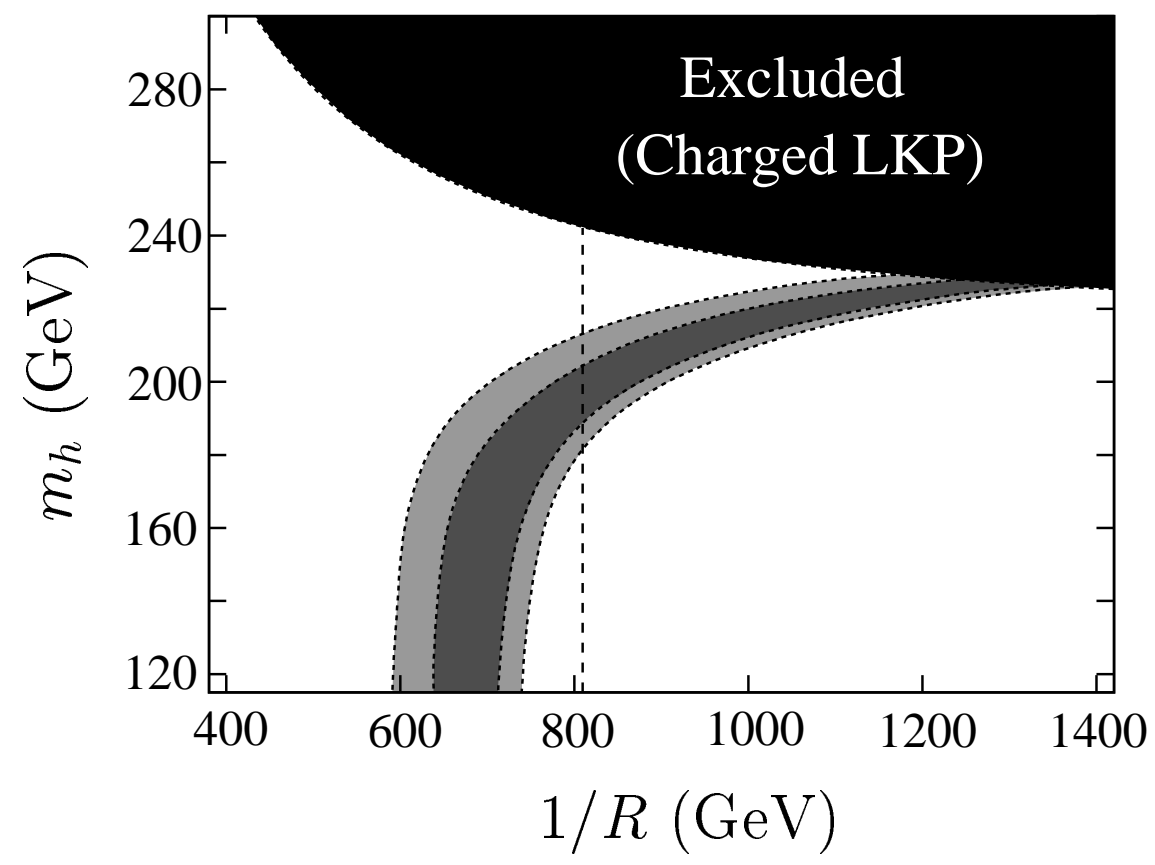

FIG. 5 Regions of minimal UED parameter space with the correct relic density. The light (medium) shaded region has $\Omega_{B^{1}} h^{2}=0.099 \pm 0.020(0.010)$. The dark shaded region is excluded because the LKP is charged. From Ref. (49).

produced through thermal freeze out and are cold and collisionless, but their implications for direct detection, indirect detection, and particle colliders may differ significantly.

\section{Direct Detection}

As discussed in Sec. III.A.3. WIMP dark matter may be detected by its scattering off normal matter through processes $X$ SM $\rightarrow X$ SM. Given a typical WIMP mass of $m_{X} \sim 100 \mathrm{GeV}$ and WIMP velocity $v \sim 10^{-3}$, the deposited recoil energy is at most $\sim 100 \mathrm{keV}$, requiring highly sensitive, low background detectors placed deep underground. Such detectors are insensitive to very strongly interacting dark matter, which would be stopped in the atmosphere or earth and would be undetectable underground. However, such dark matter would be seen by rocket and other space-borne experiments or would settle to the core of the Earth, leading to other fascinating and bizarre implications. Taken together, a diverse quilt of constraints now excludes large scattering cross sections for a wide range of dark matter masses (54; 55), and we may concentrate on the weak cross section frontier probed by underground detectors.

The field of direct detection is extremely active, with sensitivities increasing by two orders of magnitude in the last decade and bright prospects for continued rapid improvement (56, 57). WIMP scattering may be through spin-independent couplings, such as interactions $\bar{X} X \bar{q} q$, or spin-dependent couplings, such as interactions $\bar{X} \gamma^{\mu} \gamma^{5} X \bar{q} \gamma_{\mu} \gamma^{5} q$, which reduce to spin-spin couplings $S_{X} \cdot S_{q}$ in the non-relativistic limit (58). The current state of affairs is summarized in Fig. 6 for spin-independent searches and Fig. 7 for spin-dependent searches. These figures also include scattering cross section predictions for neutralino dark matter. For comparison, the predictions for $B^{1}$ Kaluza-Klein dark matter in UED for both spin- 


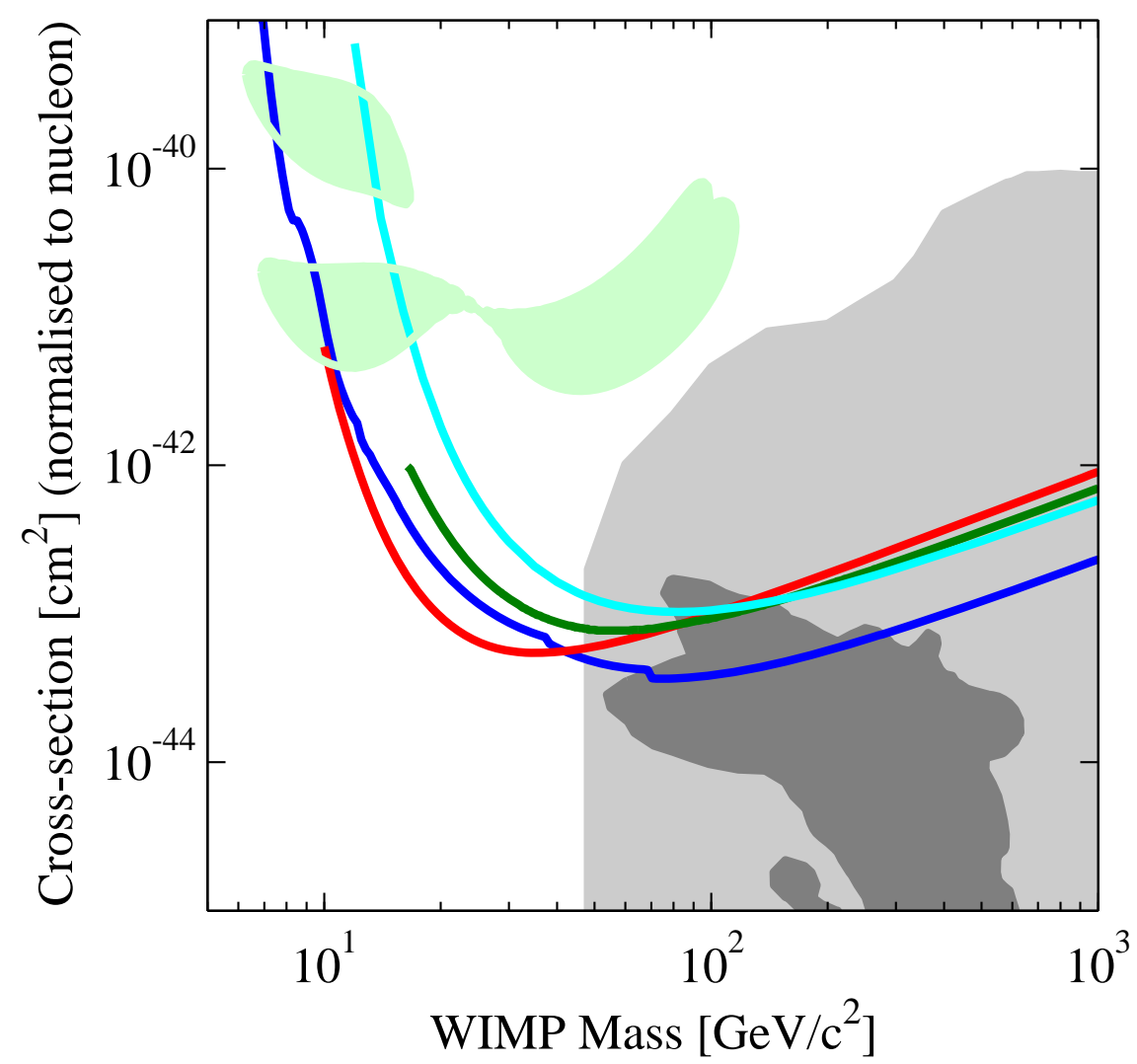

FIG. 6 Upper bounds on spin-independent WIMP-nucleon cross sections $\sigma_{\text {SI }}$ from (top to bottom at $1 \mathrm{TeV}$ WIMP mass) XENON10 (61; 62) (red), ZEPLIN III (63) (green), EDELWEISS II (64) (light blue), and CDMS II (ㅎ5) (blue), along with the combined $3 \sigma$ favored regions (green shaded) from DAMA/LIBRA (66) with and without channeling (67). The lower left shaded regions are predictions for neutralino dark matter in the general minimal supersymmetric standard model (68) (light grey) and minimal supergravity (41) (dark grey). Plot produced by DM Tools (69).

independent and spin-dependent cross sections are given in Fig. 8. These figures assume a Maxwellian velocity distribution and local dark matter density of $\rho=0.3 \mathrm{GeV} / \mathrm{cm}^{3}$; the impacts of halo modeling and Galactic substructure on direct detection limits have been explored by Ref. (59) and Ref. ([60), respectively.

For spin-independent scattering, there are both an observed signal from DAMA and null results from many other experiments. Putting aside DAMA for the moment, as can be seen in Figs. 6 and 8, current bounds exclude some of the parameter space of supersymmetry and UED, but do not test the bulk of either parameter space. The experiments are improving rapidly, however, and in the coming year, sensitivities to cross sections of $\sigma_{\mathrm{SI}} \sim 10^{-45}-$ $10^{-44} \mathrm{~cm}^{2}$ are possible.

How significant will this progress be? As evident in Fig. 6, supersymmetry predictions may be arbitrarily small. However, many well-known supersymmetric theories predict $\sigma_{\mathrm{SI}} \sim$ $10^{-44} \mathrm{~cm}^{2}$. In general, supersymmetric theories suffer from the new physics flavor problem: the introduction of squarks and sleptons with generic flavor mixing and weak scale masses induces contributions to $K-\bar{K}$ mixing, $\mu \rightarrow e \gamma$, the electric dipole moments of the neutron and electron, and a host of other flavor- or CP-violating observables that badly violate known constraints. One generic solution to this problem is to assume heavy squarks and sleptons, 


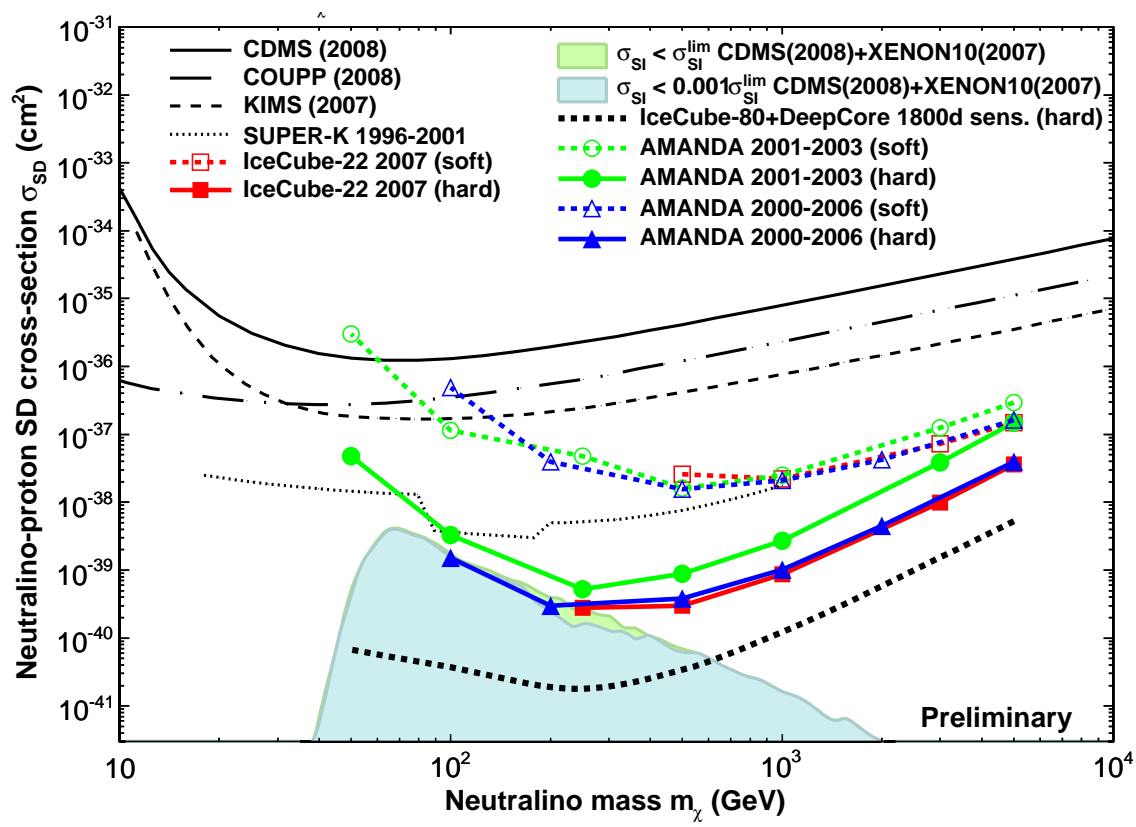

FIG. 7 Upper bounds on spin-dependent WIMP-proton cross sections $\sigma_{\mathrm{SD}}$ from CDMS (70), COUPP (71), KIMS (72), Super-K (73), and IceCube (74), along with preliminary limits from AMANDA (75) and the projected 10 year sensitivity of IceCube with DeepCore. The shaded regions are predictions for neutralino dark matter in the general minimal supersymmetric standard model with $0.05<\Omega_{\chi} h^{2}<0.20$. From Ref. (75).

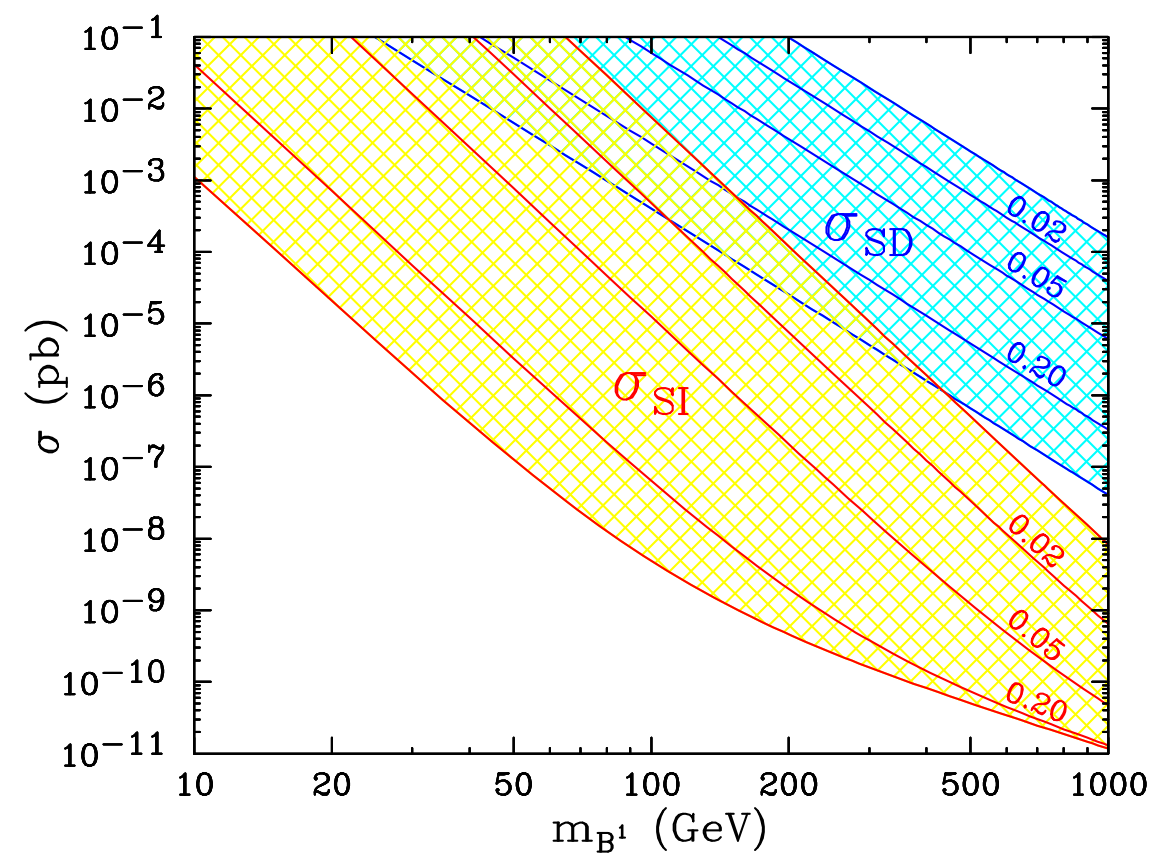

FIG. 8 Predicted spin-independent WIMP-nucleon cross sections (light shaded, red) and spindependent WIMP-proton cross sections (dark shaded, blue) in units of $\mathrm{pb}=10^{-36} \mathrm{~cm}^{2}$ for $B^{1}$ Kaluza-Klein dark matter in universal extra dimensions with a universal KK quark mass $m_{q^{1}}$. The predictions are for $m_{h}=120 \mathrm{GeV}$ and $0.01 \leq r=\left(m_{q^{1}}-m_{B^{1}}\right) / m_{B^{1}} \leq 0.5$, with contours for specific intermediate $r$ labeled. From Ref. (46). 
with masses above a $\mathrm{TeV}$, so that they decouple and do not affect low-energy observables. This solution is realized in the focus point region of minimal supergravity, and is also found in many other models with greatly varying motivations (76; 77).

These models have profound implications for dark matter searches. In general, the dominant contributions to neutralino annihilation are $\chi \chi \rightarrow q \bar{q}, l \bar{l}$ through $t$-channel squarks and sleptons, and $\chi \chi \rightarrow W^{+} W^{-}, Z Z$ through $t$-channel charginos and neutralinos. In theories with decoupled squarks and sleptons, the first class of processes are suppressed, and so annihilation takes place through the second group, which depend essentially only on the neutralino's mass and its Higgsino content. The Higgsino content may be fixed by requiring the correct thermal relic density. In these models, then, the supersymmetry parameter space is effectively reduced to one parameter, the $\chi$ mass. More detailed study shows that $\sigma_{\mathrm{SI}}$ is almost independent of $m_{\chi}$ and has a value near $10^{-44} \mathrm{~cm}^{2}(78)$.

In the next year or so, then, direct detection will test all supersymmetric scenarios with the correct relic density in which the new physics flavor problem is solved by decoupled squarks and sleptons. So far, direct detection experiments have trimmed a few fingernails off the body of supersymmetry parameter space, but if nothing is seen in the coming few years, it is arms and legs that will have been lopped off.

In addition to the limits described above, the DAMA experiment continues to find a signal in annual modulation (79) with period and maximum at the expected values (66). From a theorist's viewpoint, the DAMA/LIBRA result has been puzzling, because the signal, if interpreted as spin-independent elastic scattering, seemingly implied dark matter masses and scattering cross sections that have been excluded by other experiments. Inelastic scattering, in which dark matter is assumed to scatter through $X \mathrm{SM} \rightarrow X^{\prime} \mathrm{SM}$, where $X^{\prime}$ is another new particle that is $\sim 100 \mathrm{keV}$ heavier than $X$, has been put forward as one solution (80). More recently, astrophysics (81) and channeling (82; 83), a condensed matter effect that effectively lowers the threshold for crystalline detectors, have been proposed as possible remedies to allow elastic scattering to explain DAMA without violating other constraints. If these indications are correct, the favored parameters are $m_{X} \sim 1-10 \mathrm{GeV}$ and $\sigma_{\mathrm{SI}} \sim$ $10^{-41}-10^{-39} \mathrm{~cm}^{2}$. This interpretation is supported by unexplained events recently reported by the CoGeNT direct detection search, which, if interpreted as a dark matter signal, are best fit by dark matter with $m_{X} \sim 9 \mathrm{GeV}$ and $\sigma_{\mathrm{SI}} \sim 6.7 \times 10^{-5} \mathrm{pb}$ (84). This mass is lower than typically expected for WIMPs, but even massless neutralinos are allowed if one relaxes the constraint of gaugino mass unification (85). The cross section is, however, very large; it may be achieved in corners of minimal supersymmetric standard model (MSSM) parameter space (86), but is more easily explained in completely different frameworks, such as those discussed in Sec. VI.

Spin-dependent scattering provides an independent method to search for dark matter. At the moment, the leading direct detection experiments, such as CDMS, COUPP, and KIMS, are less promising in terms of probing the heart of supersymmetric and UED WIMP parameter space, as seen in Figs. 7 and 8. In addition, given some fairly reasonable assumptions, indirect detection experiments looking for dark matter annihilation to neutrinos in the Sun provide more stringent constraints, as we discuss in the following section.

\section{Indirect Detection}

After freeze out, dark matter pair annihilation becomes greatly suppressed. However, even if its impact on the dark matter relic density is negligible, dark matter annihilation 
continues and may be observable. Dark matter may therefore be detected indirectly: dark matter pair-annihilates somewhere, producing something, which is detected somehow. There are many indirect detection methods being pursued. Their relative sensitivities are highly dependent on what WIMP candidate is being considered, and the systematic uncertainties and difficulties in determining backgrounds also vary greatly from one method to another.

Searches for neutrinos are unique among indirect searches in that they are, given certain assumptions, probes of scattering cross sections, not annihilation cross sections, and so compete directly with the direct detection searches described in Sec. III.C. The idea behind neutrino searches is the following: when WIMPs pass through the Sun or the Earth, they may scatter and be slowed below escape velocity. Once captured, they then settle to the center, where their densities and annihilation rates are greatly enhanced. Although most of their annihilation products are immediately absorbed, neutrinos are not. Some of the resulting neutrinos then travel to the surface of the Earth, where they may convert to charged leptons through $\nu q \rightarrow \ell q^{\prime}$, and the charged leptons may be detected.

The neutrino flux depends on the WIMP density, which is determined by the competing processes of capture and annihilation. If $N$ is the number of WIMPs captured in the Earth or Sun, $\dot{N}=C-A N^{2}$, where $C$ is the capture rate and $A$ is the total annihilation cross section times relative velocity per volume. The present WIMP annihilation rate is, then, $\Gamma_{A} \equiv A N^{2} / 2=C \tanh ^{2}\left(\sqrt{C A} t_{\odot}\right) / 2$, where $t_{\odot} \simeq 4.5$ Gyr is the age of the solar system. For most WIMP models, a very large collecting body such as the Sun has reached equilibrium, and so $\Gamma_{A} \approx C / 2$. The annihilation rate alone does not completely determine the differential neutrino flux — one must also make assumptions about how the neutrinos are produced. However, if one assumes, say, that WIMPs annihilate to $b \bar{b}$ or $W^{+} W^{-}$, which then decay to neutrinos, as is true in many neutralino models, the neutrino signal is completely determined by the capture rate $C$, that is, the scattering cross section.

Under fairly general conditions, then, neutrino searches are directly comparable to direct detection. The Super-Kamiokande, IceCube, and AMANDA Collaborations have looked for excesses of neutrinos from the Sun with energies in the range $10 \mathrm{GeV} \lesssim E_{\nu} \lesssim 1 \mathrm{TeV}$. Given the assumptions specified above, their null results provide the leading bounds on spindependent scattering cross sections, as seen in Fig. 7. These experiments are just beginning to probe relevant regions of supersymmetric and UED parameter space.

Neutrino searches are also sensitive to spin-independent cross sections. For typical WIMP masses, they are not competitive with direct searches, but future neutrino searches at SuperKamiokande may have lower thresholds and so provide leading bounds on low mass WIMPs. In this way, Super-Kamiokande may test the DAMA and CoGeNT signal regions at high $\sigma_{\mathrm{SI}}$ and $m_{X} \sim 1-10 \mathrm{GeV}$ (87; 88; 89).

In addition to neutrinos, there are many other particles that may be signals of dark matter annihilation. In contrast to direct detection, there have been many reported anomalies in indirect detection, and some of these have been interpreted as possible evidence for dark matter. The most prominent recent example is the detection of positrons and electrons with energies between $10 \mathrm{GeV}$ and $1 \mathrm{TeV}$ by the PAMELA (90), ATIC (91), and Fermi LAT (92) Collaborations. These data are shown in Fig. 9, and reveal an excess above an estimate of the expected background, as modeled by GALPROP (93).

These data have several plausible astrophysical explanations. The ATIC and Fermi experiments are unable to distinguish positrons from electrons, and so constrain the total $e^{+}+e^{-}$ flux. As seen in Fig. 9, the ATIC "bump" is not confirmed by the Fermi LAT data, which has much higher statistics. The Fermi data may be explained by modifying the spectral 

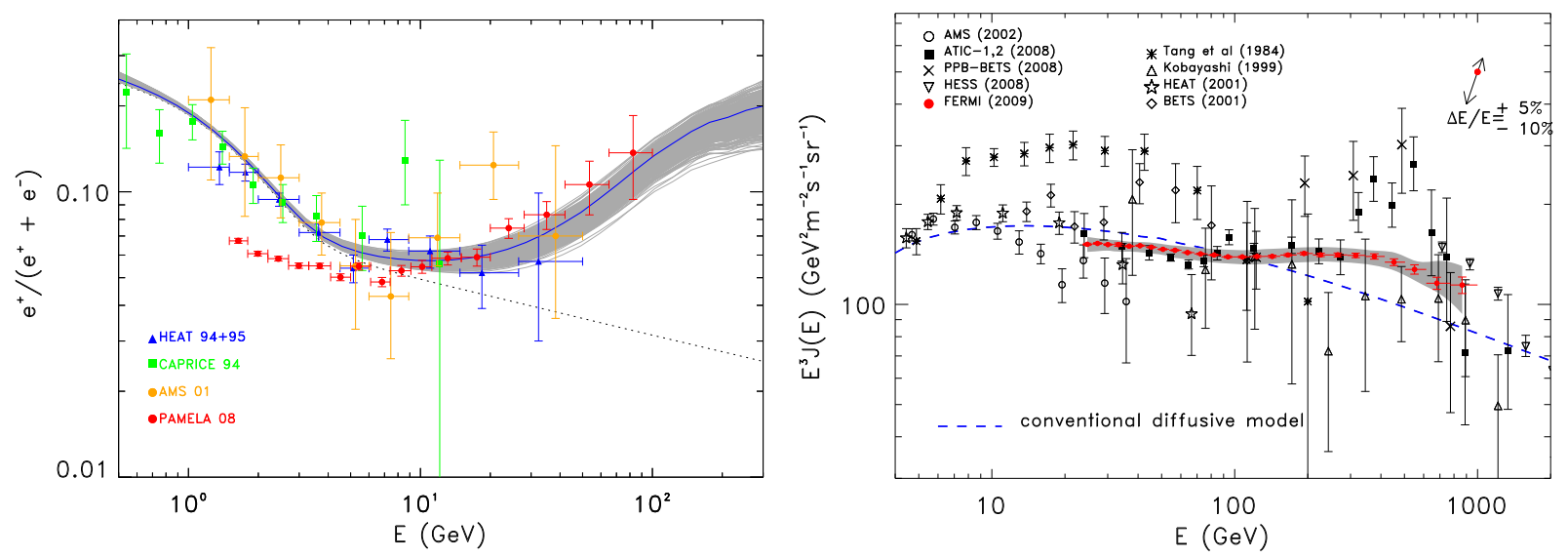

FIG. 9 Left: the cosmic positron fraction measured by PAMELA and other experiments and the predictions of pulsars with various parameters (grey contours) (94). Discrepancies at energies below $10 \mathrm{GeV}$ are claimed to arise from solar modulation. Right: the total $e^{+}+e^{-}$flux measured by ATIC, Fermi, and other experiments (92). In both cases, the dashed contours represent the predicted backgrounds from GALPROP (93).

index of the cosmic ray background (94). This exacerbates the PAMELA discrepancy, but the PAMELA data, with or without the modified spectral index, is consistent with expectations from pulsars derived both before $($ 95, 96) and after $(97,98,99)$ the PAMELA data (see Fig. 9), and may also have other astrophysical explanations (100; 101; 102).

Despite the astrophysical explanations, one may explore the possibility that the positron excesses arise from dark matter annihilation. The energies of the excess, around $m_{\text {weak }}$, are as expected for WIMPs. Unfortunately, the observed fluxes are far larger than expected for generic WIMPs. For a WIMP annihilating through $S$-wave processes to have the desired thermal relic density, its annihilation cross section must be $\sigma^{\text {th }} \equiv\left\langle\sigma_{A} v\right\rangle \approx 3 \times 10^{-26} \mathrm{~cm}^{3} / \mathrm{s}$. This must be enhanced by two or three orders of magnitudes to explain the positron data, as shown in Fig. 10. Astrophysical boosts from substructure are unable to accommodate such large enhancements, and so one must look to particle physics.

A seemingly attractive solution is to postulate that dark matter interacts with a light force carrier $\phi$ with fine structure constant $\alpha_{X} \equiv \lambda^{2} /(4 \pi)$. For massless $\phi$, this enhances the annihilation cross section by the Sommerfeld enhancement factor

$$
S=\frac{\pi \alpha_{X} / v_{\mathrm{rel}}}{1-e^{-\pi \alpha_{X} / v_{\mathrm{rel}}}}
$$

an effect first derived for the case of $e^{+} e^{-}$annihilation (105). For massive $\phi, S$ is typically cut off at a value $\propto \alpha_{X} m_{X} / m_{\phi}$ (106; 107; 108; 109). The relative velocity of colliding dark matter particles is $v_{\text {rel }} \sim 1 / 3$ at freeze out and $v_{\text {rel }} \sim 10^{-3}$ now. The Sommerfeld enhancement therefore provides an elegant mechanism for boosting annihilations now. The case $m_{\phi}=0$ is excluded by constraints from dark matter annihilation in protohalos with $v_{\text {rel }} \sim 10^{-8}$ (110). However, taking $m_{X} \sim \mathrm{TeV}$ and $m_{\phi} \sim \mathrm{MeV}-\mathrm{GeV}$, and assuming $\left\langle\sigma_{A} v_{\text {rel }}\right\rangle \approx \sigma^{\text {th }}$, one may seemingly still generate $S \sim 10^{3}$ to explain the positron excesses, while the cutoff allows one to satisfy the protohalo constraint.

Unfortunately, for the annihilation cross section for $X X \rightarrow \phi \phi$ to give the correct relic density, $\alpha_{X}$ cannot be too large, which bounds $S$ from above. Even ignoring the $S$ cutoff for massive $\phi$, the resulting constraints exclude the possibility that, with standard astrophysical 


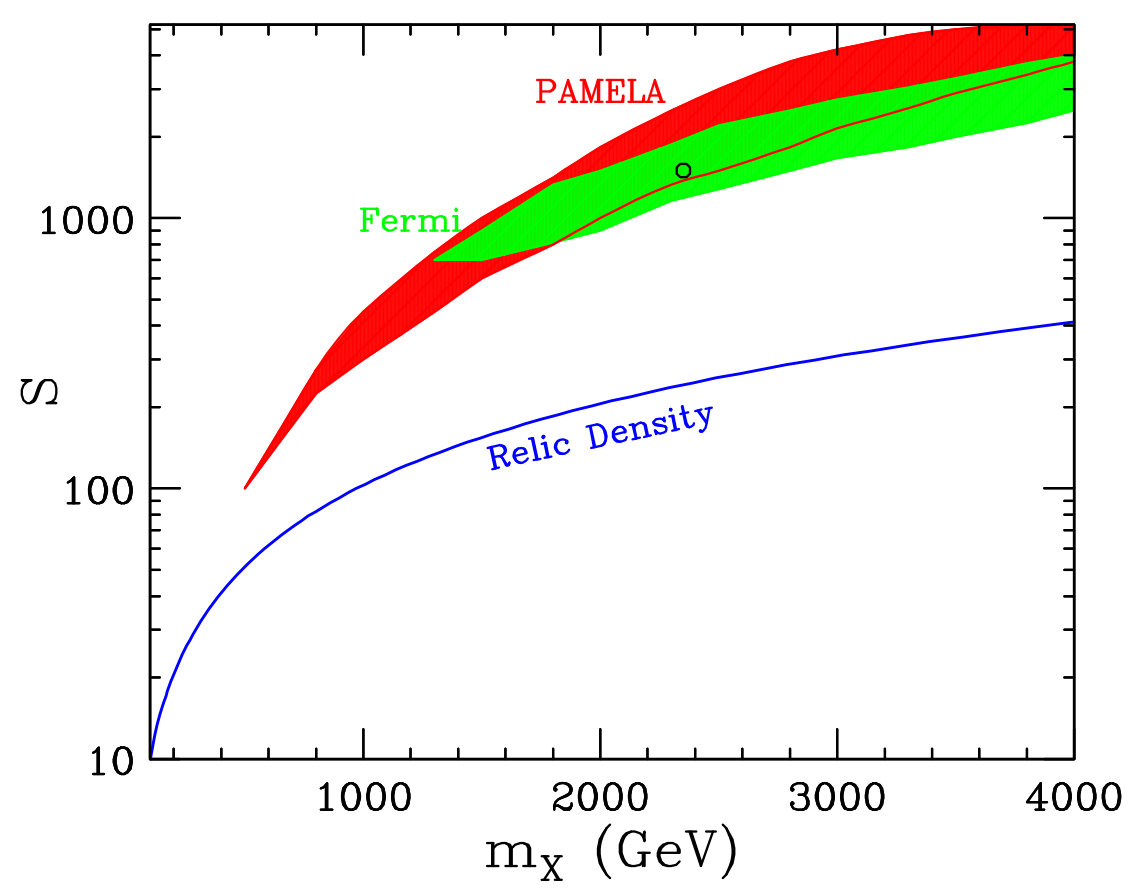

FIG. 10 The annihilation cross section enhancement factor $S$ required to explain the PAMELA and Fermi data, as a function of dark matter mass $m_{X}$ (shaded regions) (103), along with upper bounds on $S$ from the requirement that the dark matter have the right thermal relic density. From Ref. (104).

assumptions, Sommerfeld enhancement alone can explain the PAMELA and Fermi excesses, as shown in Fig. 10. For particular choices of $\alpha_{X}, m_{X}$ and $m_{\phi}, S$ may in fact be resonantly enhanced, but these enhancements also reduce the thermal relic density (111; 112); including the effect on the relic density in fact increases the discrepancy significantly.

There are other proposed dark matter explanations: for example, the annihilation cross section may be boosted by resonances from states with mass $\sim 2 m_{X}(113$; 114; 115; 116), or the dark matter may be produced not by thermal freeze out, but by decays (117). At present, however, the dark matter explanations are considerably more exotic than the astrophysical ones. Additional data from, for example, Fermi and the Alpha Magnetic Spectrometer (AMS), an anti-matter detector to be placed on the International Space Station, may be able to distinguish the various proposed explanations for the positron excesses, as well as be sensitive to canonical WIMP models, but it remains to be seen whether the astrophysical backgrounds may be sufficiently well understood for these experiments to realize their dark matter search potential.

In addition to neutrinos from the Sun and positrons from the galactic halo, there are several other promising indirect detection search strategies. Searches for anti-protons and anti-deuterons from WIMP annihilation in the galactic halo provide complementary searches, as they are sensitive to dark matter candidates that annihilate primarily to quarks. In addition, searches for gamma rays by space-based experiments, such as Fermi and AMS, and by ground-based atmospheric Cherenkov telescopes are also promising. The most striking gamma ray signal would be mono-energetic photons from $X X \rightarrow \gamma \gamma$, but since WIMPs cannot be charged, these processes are typically loop-induced or otherwise highly suppressed. More commonly, gamma rays are produced when WIMPs annihilate to other particles, which 
then radiate photons, leading to a smooth distribution of gamma ray energies. On the other hand, photons point back to their source, providing a powerful diagnostic. Possible targets for gamma ray searches are the center of the Galaxy, where signal rates are high but backgrounds are also high and potentially hard to estimate, and dwarf galaxies, where signal rates are lower, but backgrounds are also expected to be low.

\section{E. Particle Colliders}

If WIMPs are the dark matter, what can colliders tell us? Given the energy of the LHC and the requirement that WIMPs have mass $\sim m_{\text {weak }}$ and interact through the weak force, WIMPs will almost certainly be produced at the LHC. Unfortunately, direct WIMP production of $X X$ pairs is invisible. The next best targets are mono-jet or mono-photon signals from $X X j$ and $X X \gamma$ production, respectively, where the jet $j$ or photon comes from initial state radiation. At the International Linear Collider, a proposed high energy $e^{+} e^{-}$collider, such signals can been disentangled from background, using the fact that the initial state particles have definite energy and may be polarized, which provides a useful diagnostic (118). Unfortunately, these features are missing at hadron colliders, where the initial state protons have fixed energy but the quarks and gluons do not. As a result, at the Tevatron and LHC, the mono-jet and mono-photon signals are completely obscured by backgrounds such as $Z j$ and $Z \gamma$ followed by $Z \rightarrow \nu \bar{\nu}(119)$.

Searches for dark matter at the LHC therefore rely on indirect production. For example, in supersymmetry, the LHC will typically produce pairs of squarks and gluinos. These will then decay through some cascade chain, eventually ending up in neutralino WIMPs, which escape the detector. Their existence is registered through the signature of missing energy and momentum, a signal that is a staple of searches for physics beyond the SM.

Although the observation of missing particles is consistent with the production of dark matter, it is far from compelling evidence. The observation of missing particles only implies that a particle was produced that was stable enough to exit the detector, typically implying a lifetime $\tau \gtrsim 10^{-7} \mathrm{~s}$, a far cry from the criterion $\tau \gtrsim 10^{17} \mathrm{~s}$ required for dark matter.

Clearly more is needed. In the last few years, there has been a great deal of progress in this direction. The main point of this progress has been to show that colliders can perform detailed studies of new physics, and this can constrain the dark matter candidate's properties so strongly that the candidate's thermal relic density can be precisely determined. The consistency of this density with the cosmologically observed density would then be strong evidence that the particle produced at colliders is, in fact, the cosmological dark matter.

This approach is analogous to the well-known case of BBN. For BBN, data from nuclear physics experiments stringently constrain cross sections involving the light nuclei. Along with the assumption of a cooling and expanding Universe, this allows one to predict the light element abundances left over from the Big Bang, and the consistency of these predictions with observations gives us confidence that the light elements were actually created in this way. For dark matter, the idea is that particle physics experiments at the LHC may stringently constrain cross sections involving dark matter and related particles. Along with the assumption of a cooling and expanding Universe, this microscopic data allows one to predict the dark matter relic density, basically by following the relic density curves of Fig. 2 . This thermal relic density may be compared to the observed density of dark matter, and their consistency would give us confidence that dark matter is actually produced in this way and is made of the particles produced at the collider. 


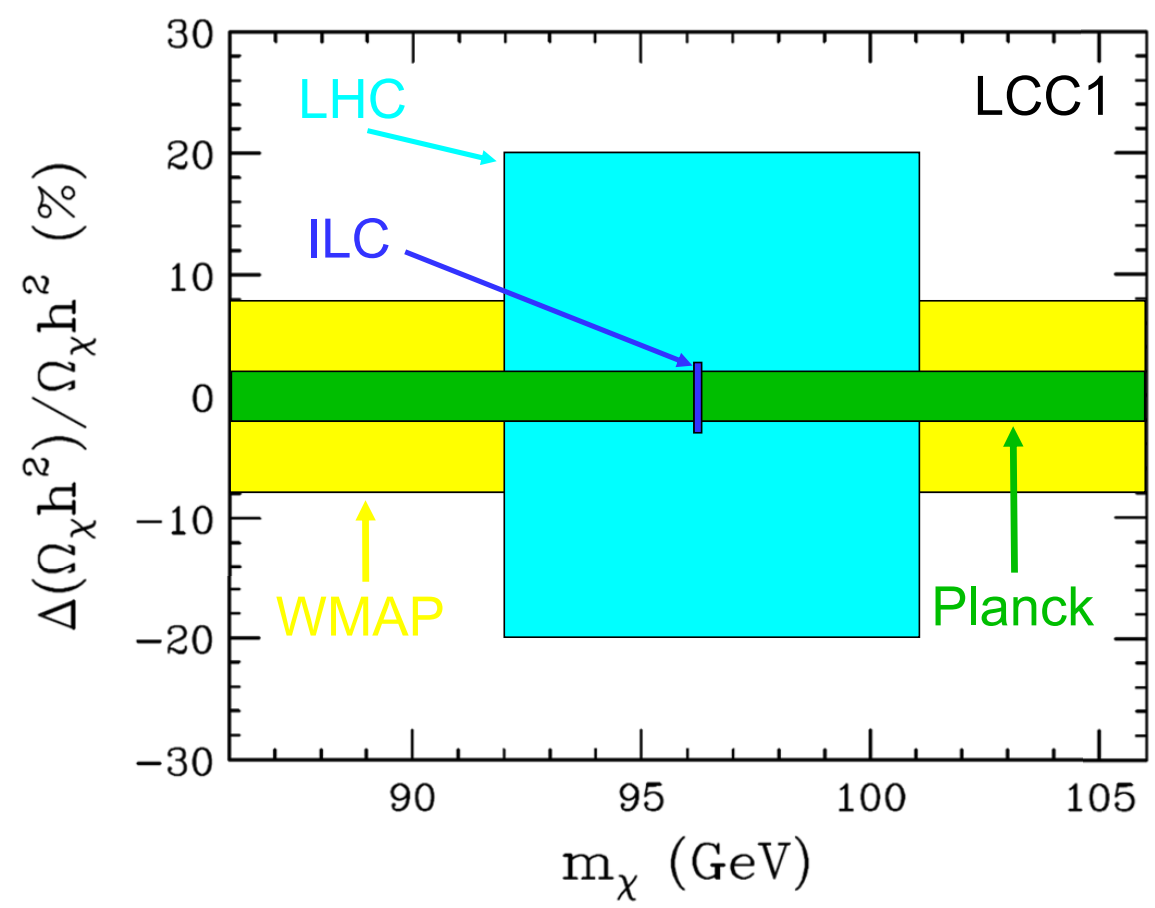

FIG. 11 Constraints in the $\left(m_{\chi}, \Omega_{\chi}\right)$ plane from the LHC and the International Linear Collider, and from the WMAP and Planck satellites (123; 124). WMAP and Planck measure $\Omega_{\chi}$, but are insensitive to the dark matter mass $m_{\chi}$; the collider experiments bound both. These results are for LCC1, a supersymmetric model with minimal supergravity parameters $\left(m_{0}, M_{1 / 2}, \tan \beta, A_{0}, \operatorname{sign}(\mu)\right)=(100 \mathrm{GeV}, 250 \mathrm{GeV}, 10,-100 \mathrm{GeV},+)$.

How well can the LHC do? The answer depends sensitively on the underlying dark matter scenario, but several qualitatively different cases have now been studied $(120 ; 121 ; 122$; 123 ). The results of one (exemplary) case study are given in Fig. 11. In conjunction with other cosmological observations, the WMAP satellite constrains the dark matter relic density $\Omega_{\chi}$ to a fractional uncertainty of $\pm 8 \%$. Its successor, Planck, is expected to sharpen this to $\pm 2 \%$. Of course, $\mathrm{CMB}$ experiments do not constrain the dark matter mass. At the same time, precision studies at the LHC can determine so many of the supersymmetric model parameters that the neutralino mass can be determined to $\pm 5 \mathrm{GeV}$ and the thermal relic density can be predicted to $\pm 20 \%$. Measurements at the International Linear Collider could improve these constraints on mass and relic density to $\pm 50 \mathrm{MeV}$ and $\pm 3 \%$, respectively.

Consistency between the particle physics predictions and the cosmological observations would provide compelling evidence that the particle produced at the LHC is in fact dark matter. Along the way, the colliders will also determine the dark matter's mass, spin, and many other properties. In this way, colliders may finally help solve the question of the microscopic identity of dark matter. Note also that, just as BBN gives us confidence that we understand the Universe back to 1 second after the Big Bang and temperatures of $1 \mathrm{MeV}$, such dark matter studies will provide a window on the era of dark matter freeze out at 1 nanosecond after the Big Bang and temperatures of $\sim 10 \mathrm{GeV}$. Of course, the thermal relic density prediction from colliders and the cosmological observations need not be consistent. In this case, there are many possible lines of inquiry, depending on which is larger. 


\section{SUPERWIMPS}

The WIMP miracle might appear to require that dark matter have weak interactions if its relic density is naturally to be in the right range. This is not true, however - in recent years, two other mechanisms have been shown to be viable and lead to dark matter particles that also exploit the WIMP miracle to have the correct relic density, but have vastly different interactions and implications for detection. These two possibilities are the topics of this section and Sec. VI.

In this section, we discuss superWIMPs, superweakly-interacting massive particles, which have the desired relic density, but have interactions that are much weaker than weak. The extremely weak interactions of SuperWIMPs might naively be thought to be a nightmare for searches for dark matter. In fact, superWIMP scenarios predict signals from cosmic rays, at colliders, and in astrophysics that can be far more striking than in WIMP scenarios, making superWIMPs highly amenable to experimental investigation.

\section{A. Production Mechanisms}

\section{DECAYS}

In the superWIMP framework, dark matter is produced in late decays: WIMPs freeze out as usual in the early Universe, but later decay to superWIMPs, which form the dark matter that exists today (125; 126). Because superWIMPs are very weakly interacting, they have no impact on WIMP freeze out in the early Universe, and the WIMPs decouple, as usual, with a thermal relic density $\Omega_{\text {WIMP }} \sim \Omega_{\text {DM }}$. Assuming that each WIMP decay produces one superWIMP, the relic density of superWIMPs is

$$
\Omega_{\mathrm{SWIMP}}=\frac{m_{\mathrm{SWIMP}}}{m_{\mathrm{WIMP}}} \Omega_{\mathrm{WIMP}} .
$$

SuperWIMPs therefore inherit their relic density from WIMPs, and for $m_{\text {SWIMP }} \sim m_{\text {WIMP }}$, the WIMP miracle also implies that superWIMPs are produced in the desired amount to be much or all of dark matter. The evolution of number densities is shown in Fig. 12 , The WIMP decay may be very late by particle physics standards. For example, if the superWIMP interacts only gravitationally, as is true of many well-known candidates, the natural timescale for WIMPs decaying to superWIMP is $1 /\left(G_{N} m_{\text {weak }}^{3}\right) \sim 10^{3}-10^{7} \mathrm{~s}$.

Because the WIMP is unstable and not the dark matter, it need not be neutral in this context - to preserve the naturalness of the superWIMP relic density, all that is required is $\Omega_{\text {WIMP }} \sim \Omega_{\mathrm{DM}}$. In the case of supersymmetry, for example, the WIMP may be a neutralino, but it may also be a charged slepton. Even though charged sleptons interact with photons, on dimensional grounds, their annihilation cross sections are also necessarily governed by

the weak scale, and so are $\sim g_{\text {weak }}^{4} / m_{\text {weak }}^{2}$, implying roughly the same relic densities as their neutral counterparts. Of course, whether the WIMP is charged or not determines the properties of the other particles produced in WIMP decay, which has strong consequences for observations, as we will see below.

\section{REHEATING}

SuperWIMPs may also be produced after reheating, when the energy of the inflaton potential is transferred to SM and other particles. This creates a hot thermal bath, and, if 


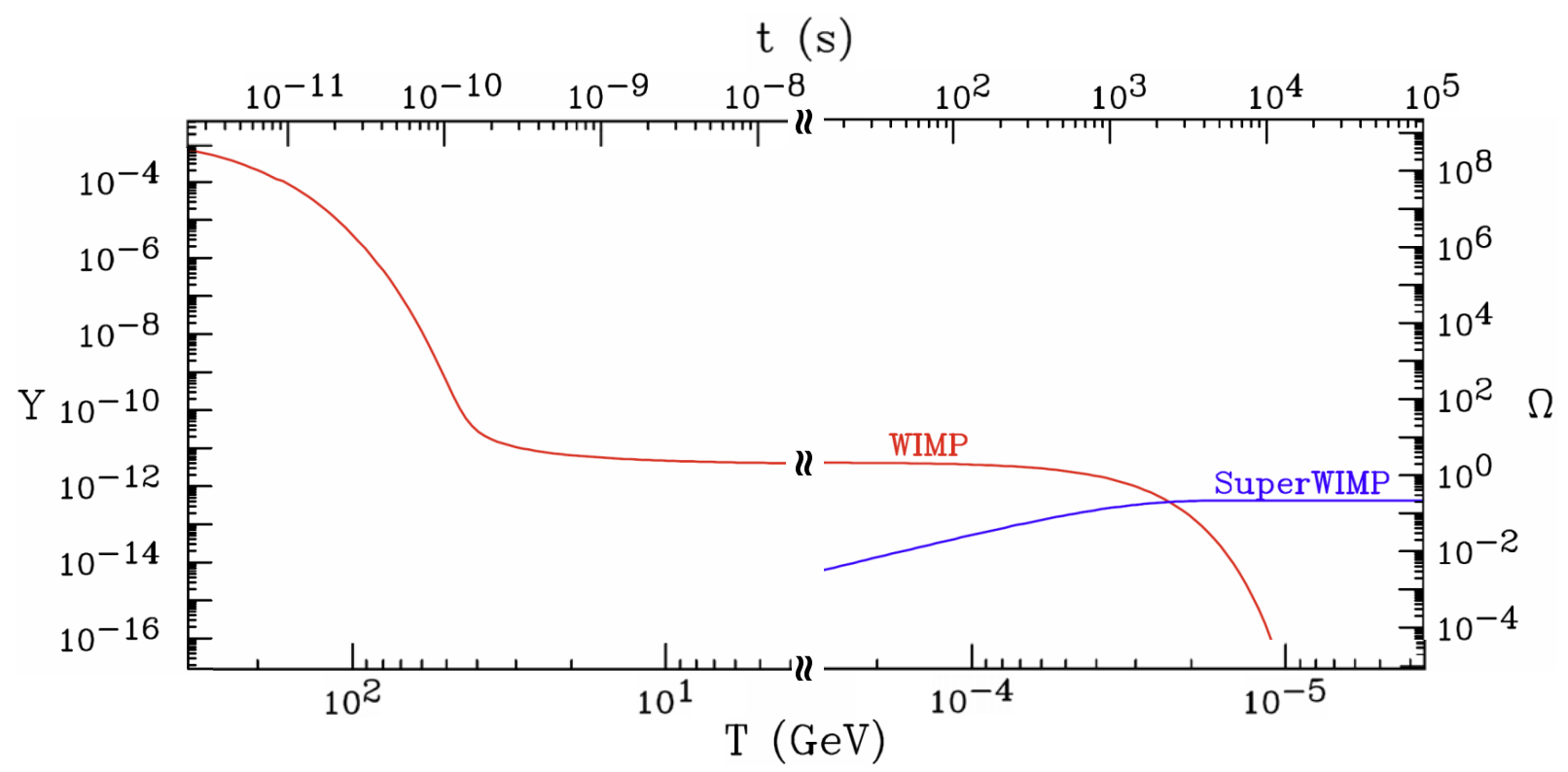

FIG. 12 In superWIMP scenarios, WIMPs freeze out as usual, but then decay to superWIMPs, superweakly-interacting massive particles that are the dark matter. This figure shows the WIMP comoving number density $Y$ (left) and the superWIMP relic density (right) as functions of temperature $T$ (bottom) and time $t$ (top). The WIMP is a $1 \mathrm{TeV}, S$-wave annihilating particle with lifetime $10^{3} \mathrm{~s}$, and the superWIMP has mass $100 \mathrm{GeV}$.

the temperature is high enough, may be a significant source of superWIMPs (127; 128, 129, 130; 131).

After reheating, the Universe is characterized by three rates: the interaction rate of particles that have SM interactions, $\sigma_{\mathrm{SM}} n$; the expansion rate, $H$; and the rate of interactions involving a superWIMP, $\sigma_{\mathrm{SWIMP}} n$. Here $n$ is the number density of SM particles. After reheating, the Universe is expected to have a temperature well below the Planck scale, but still well above SM masses. Assuming the superWIMP is gravitationally interacting, dimensional analysis implies that these rates are hierarchically separated:

$$
\sigma_{\mathrm{SM}} n \sim T \gg H \sim \frac{T^{2}}{M_{\mathrm{Pl}}} \gg \sigma_{\mathrm{SWIMP}} n \sim \frac{T^{3}}{M_{\mathrm{Pl}}^{2}} .
$$

This implies a very simple picture: after reheating, particles with SM interactions are in thermal equilibrium with each other. Occasionally these interact to produce a superWIMP. The produced superWIMPs then propagate through the Universe essentially without interacting and without annihilating, surviving to the present day.

To determine the superWIMP abundance, we turn once again to the Boltzmann equation of Eq. (5), where now $n$ is the number density of superWIMPs. To be concrete, consider the case of gravitino superWIMPs. In this case, the source term $n_{\text {eq }}^{2}$ arises from interactions that produce superWIMPs, such as $g g \rightarrow \tilde{g} \tilde{G}$. In contrast to our previous application of the Boltzmann equation in Sec. III.A.1, however, here the sink term $n^{2}$ is negligible. Changing variables with $t \rightarrow T$ and $n \rightarrow Y \equiv n / s$, where $s$ is the entropy density, we find

$$
\frac{d Y}{d T}=-\frac{\left\langle\sigma_{\tilde{G}} v\right\rangle}{H T s} n^{2}
$$


The right-hand side is independent of $T$, since $n \propto T^{3}, H \propto T^{2}$ and $s \propto T^{3}$. We thus find an extremely simple relation - the superWIMP relic number density is linearly proportional to the reheating temperature $T_{R}$.

The constant of proportionality is the gravitino production cross section. Including all such production mechanisms, the gravitino relic density can be determined as a function of reheating temperature $T_{R}$ and gravitino mass. For gravitino mass $m_{\tilde{G}} \sim 100 \mathrm{GeV}$, the constraint on $\Omega_{\mathrm{DM}}$ requires $T_{R} \lesssim 10^{10} \mathrm{GeV}$ (132). Of course, if this bound is nearly saturated, gravitinos produced after reheating may be a significant component of dark matter, adding to the relic density from decays.

\section{B. Candidates}

\section{WEAK-SCALE GRAVITINOS}

The superWIMP scenario is realized in many particle physics models. The prototypical superWIMP is the gravitino $\tilde{G}$ (125; 126; 133; 134; 135; 136). Gravitinos are the spin $3 / 2$ superpartners of gravitons, and they exist in all supersymmetric theories. The gravitino's mass is

$$
m_{\tilde{G}}=\frac{F}{\sqrt{3} M_{*}},
$$

where $F$ is the supersymmetry-breaking scale squared and $M_{*}=\left(8 \pi G_{N}\right)^{-1 / 2} \simeq 2.4 \times$ $10^{18} \mathrm{GeV}$ is the reduced Planck mass. In the simplest supersymmetric models, where supersymmetry is transmitted to SM superpartners through gravitational interactions, the masses of SM superpartners are

$$
\tilde{m} \sim \frac{F}{M_{*}} .
$$

A solution to the gauge hierarchy problem requires $F \sim\left(10^{11} \mathrm{GeV}\right)^{2}$, and so all superpartners and the gravitino have weak-scale masses. The precise ordering of masses depends on unknown, presumably $\mathcal{O}(1)$, constants in Eq. (21). There is no theoretical reason to expect the gravitino to be heavier or lighter than the lightest SM superpartner, and so in roughly "half" of the parameter space, the gravitino is the lightest supersymmetric particle (LSP). Its stability is guaranteed by $R$-parity conservation, and since $m_{\tilde{G}} \sim \tilde{m}$, the gravitino relic density is naturally $\Omega_{\text {SWIMP }} \sim \Omega_{\mathrm{DM}}$.

In gravitino superWIMP scenarios, the role of the decaying WIMP is played by the next-to-lightest supersymmetric particle (NLSP), a charged slepton, sneutrino, chargino, or neutralino. The gravitino couples SM particles to their superpartners through gravitinosfermion-fermion interactions

$$
L=-\frac{1}{\sqrt{2} M_{*}} \partial_{\nu} \tilde{f} \bar{f} \gamma^{\mu} \gamma^{\nu} \tilde{G}_{\mu}
$$

and gravitino-gaugino-gauge boson interactions

$$
L=-\frac{i}{8 M_{*}} \overline{\tilde{G}}_{\mu}\left[\gamma^{\nu}, \gamma^{\rho}\right] \gamma^{\mu} \tilde{V} F_{\nu \rho}
$$

The presence of $M_{*}$ in Eqs. (22) and (23) implies that gravitinos interact only gravitationally, a property dictated by the fact that they are the superpartners of gravitons. These 
interactions determine the NLSP decay lifetime. As an example, if the NLSP is a stau, a superpartner of the tau lepton, its lifetime is

$$
\tau(\tilde{\tau} \rightarrow \tau \tilde{G})=\frac{6}{G_{N}} \frac{m_{\tilde{G}}^{2}}{m_{\tilde{\tau}}^{5}}\left[1-\frac{m_{\tilde{G}}^{2}}{m_{\tilde{\tau}}^{2}}\right]^{-4} \approx 3.6 \times 10^{7} \mathrm{~s}\left[\frac{100 \mathrm{GeV}}{m_{\tilde{\tau}}-m_{\tilde{G}}}\right]^{4}\left[\frac{m_{\tilde{G}}}{100 \mathrm{GeV}}\right]
$$

where the approximate expression holds for $m_{\tilde{G}} / m_{\tilde{\tau}} \approx 1$. We see that decay lifetimes of the order of hours to months are perfectly natural. At the same time, the lifetime is quite sensitive to the underlying parameters and may be much longer for degenerate $\tilde{\tau}-\tilde{G}$ pairs, or much shorter for light gravitinos.

\section{OTHERS}

In addition to gravitinos, other well-motivated examples of superWIMPs include axinos (137; 138; 139), the supersymmetric partners of axions, particles introduced to resolve the strong CP problem described in Sec. II.B.4. Axions may also be cold dark matter (see Sec. VIII), and the possibility that both axions and axinos contribute to dark matter is one of the better motivated multi-component dark matter scenarios (140). UED models also have superWIMP candidates in the form of Kaluza-Klein graviton and axion states (141). One of these may be the lightest KK state; in fact, in minimal UED, the KK graviton is the lightest $\mathrm{KK}$ state for all $R^{-1}<800 \mathrm{GeV}$, where $R$ is the compactification radius (142). If one of these is the lightest KK state, it is stabilized by KK-parity conservation and has properties very similar to its supersymmetric analogue. Other superWIMPs candidates include quintessinos in supersymmetric theories (143), stable particles in models that simultaneously address the problem of baryon asymmetry (144), and other particles produced in decays, where the decay time is greatly lengthened by mass degeneracies (145, 146).

In summary, there are many superWIMP candidates that possess all of the key virtues of WIMPs: they exist in the same well-motivated frameworks and, since they inherit their relic density from WIMPs, are also naturally produced with the desired relic density. As we will see, however, they have completely different implications for detection.

\section{Indirect Detection}

SuperWIMPs are so weakly interacting that they cannot be detected by direct searches, and their annihilation cross sections are so suppressed that their annihilation signal rates are completely negligible. However, if the decaying WIMP is charged, the superWIMP scenario implies long-lived charged particles, with striking implications for indirect detection.

One interesting possibility is that long-lived charged particles may be produced by ultrahigh energy cosmic rays, resulting in exotic signals in cosmic ray and cosmic neutrino experiments (147; 148). As an example, in the gravitino superWIMP scenario with a stau NLSP, ultra-high energy neutrinos may produce events with two long-lived staus through $\nu q \rightarrow \tilde{\tau} \tilde{q}^{\prime}$ followed by the decay $\tilde{q}^{\prime} \rightarrow \tilde{\tau}$. The metastable staus then propagate to neutrino telescopes (149), where they have a typical transverse separation of hundreds of meters. They may therefore be detected above background as events with two upward-going, extremely high energy charged tracks in experiments such as IceCube. 


\section{Particle Colliders}

Particle colliders may also find evidence for superWIMP scenarios. This evidence may come in one of two forms. Collider experiments may see long-lived, charged particles. Given the stringent bounds on charged dark matter, such particles presumably decay, and their decay products may be superWIMPs. Alternatively, colliders may find seemingly stable particles, but the precision studies described in Sec. III.E may imply that these particles have a thermal relic density that is too large. These two possibilities are not mutually exclusive. In fact, the discovery of long-lived, charged particles with too-large predicted relic density is a distinct possibility and would strongly motivate superWIMP dark matter.

As an example, again consider gravitino superWIMPs with a charged slepton NLSP. (The possibility of a neutralino NLSP is essentially excluded by considerations of BBN, as discussed in Sec. IV.E.2.) In the slepton NLSP scenario, supersymmetric events at colliders are not characterized by missing energy and momentum as predicted in WIMP dark matter scenarios, but rather by pairs of heavy, long-lived, charged particles. Such particles lead to spectacular signals and require far less luminosity for discovery than missing energy signals (150; 151).

To determine the superWIMP relic density of Eq. (17), we must determine the superWIMP's mass. This is not easy, since the WIMP lifetime may be very large, implying that superWIMPs are typically produced long after the WIMPs have escaped collider detectors. At the same time, because some sleptons will be slowly moving and highly-ionizing, they may be trapped and studied. As an example, sleptons may be trapped in water tanks placed outside collider detectors. These water tanks may then be drained periodically to underground reservoirs where slepton decays can be observed in quiet environments. The number of sleptons that may be trapped is model-dependent, but a 1 meter thick tank of water may trap as many as a thousand sleptons per year (152). Other possibilities for capturing sleptons include using the LHC detectors themselves as the slepton traps (153), or carefully tracking sleptons as they exit the detector and digging them out of the walls of the detector halls, giving new meaning to the phrase "data mining" (154).

If thousands of sleptons are trapped, the slepton lifetime may be determined to the few percent level simply by counting the number of slepton decays as a function of time. The slepton mass will be constrained by analysis of the collider event kinematics. Furthermore, the outgoing lepton energy can be measured, and this provides a high precision measurement of the gravitino mass, and therefore a determination of the gravitino relic density through Eq. (17). Consistency at the percent level with the observed dark matter relic density will provide strong evidence that dark matter is indeed composed of gravitino superWIMPs.

Perhaps as interesting, the determination of $\tau, m_{\tilde{G}}$, and $m_{\tilde{l}}$ in Eq. (24) implies that one can differentiate the various superWIMP candidates (155) and determine Newton's constant on the scale of fundamental particles (134; 156). According to conventional wisdom, particle colliders are insensitive to gravity, since it is such a weak force. We see that this is not true - if $G_{N}$ enters in a decay time, one can achieve the desired sensitivity simply by waiting a long time. In this case, one can measure the force of gravity between two test particles with masses $\sim 10^{-27} \mathrm{~kg}$, a regime that has never before been probed. If this force is consistent with gravity, these studies will show that the newly discovered particle is indeed interacting gravitationally, as is required for the gravitino to be the graviton's superpartner, and demonstrate that gravity is in fact extended to supergravity in nature. 


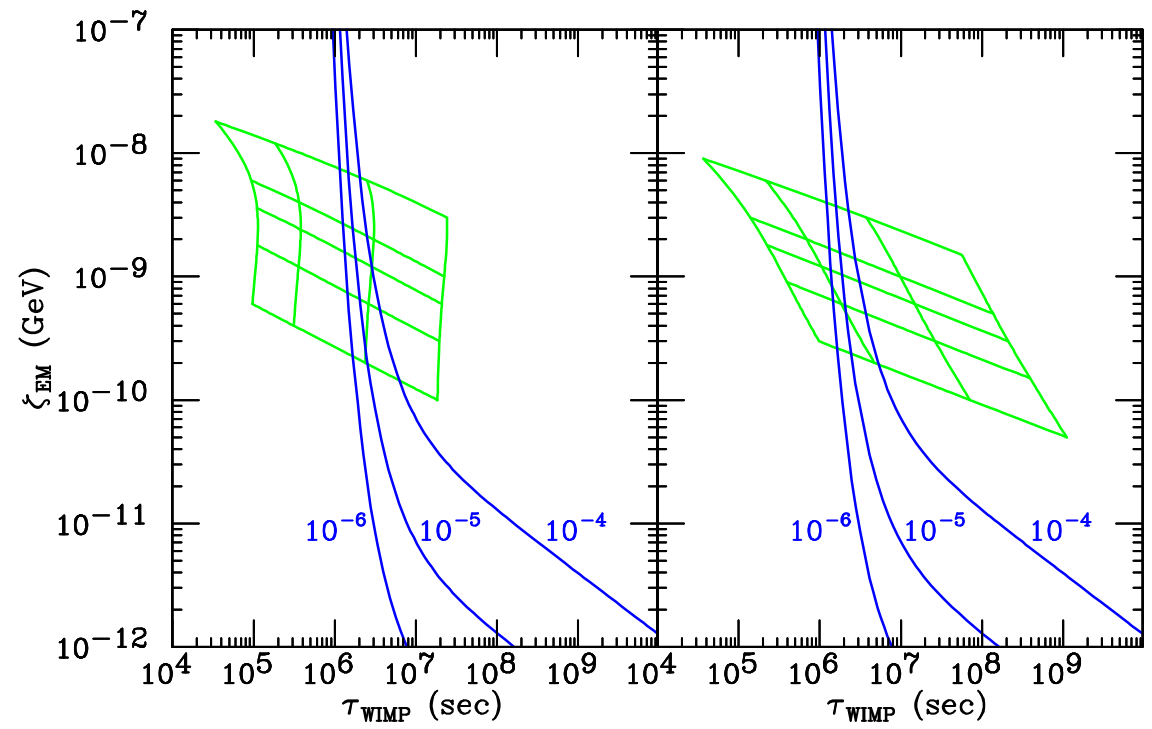

FIG. 13 Predicted values of WIMP lifetime $\tau$ and electromagnetic energy release $\zeta_{\mathrm{EM}} \equiv \varepsilon_{\mathrm{EM}} Y_{\mathrm{NLSP}}$ in the $\tilde{B}$ (left) and $\tilde{\tau}$ (right) superWIMP scenarios for $m_{\text {SWIMP }}=100 \mathrm{GeV}, 300 \mathrm{GeV}, 500 \mathrm{GeV}$, $1 \mathrm{TeV}$, and $3 \mathrm{TeV}$ (top to bottom) and $\Delta m \equiv m_{\mathrm{WIMP}}-m_{\mathrm{SWIMP}}=600 \mathrm{GeV}, 400 \mathrm{GeV}, 200 \mathrm{GeV}$, and $100 \mathrm{GeV}$ (left to right). The contours are for $\mu$, which parameterizes the distortion of the CMB from a Planckian spectrum. From Ref. (126).

\section{E. Astrophysical Signals}

Because superWIMPs are very weakly interacting, the decays of WIMPs to superWIMPs may be very late and have an observable effects on BBN and the CMB. In addition, in contrast to WIMPs, superWIMP dark matter may behave as warm dark matter.

\section{COSMIC MICROWAVE BACKGROUND}

When WIMPs decay to superWIMPs, the accompanying particles may distort the frequency dependence of the CMB away from its ideal black body spectrum (126; 157; 158). The impact on the CMB is determined by the NLSP lifetime and the energy released in visible decay products when the WIMP decays. The energy release is conveniently expressed in terms of

$$
\zeta_{\mathrm{EM}} \equiv \epsilon_{\mathrm{EM}} Y_{\mathrm{NLSP}}
$$

for electromagnetic energy, with a similar expression for hadronic energy. Here $\epsilon_{\mathrm{EM}}$ is the initial EM energy released in NLSP decay and $Y_{\mathrm{NLSP}} \equiv n_{\mathrm{NLSP}} / n_{\gamma}$ is the NLSP number density just before it decays, normalized to the background photon number density $n_{\gamma}=$ $2 \zeta(3) T^{3} / \pi^{2}$. Once the NLSP is specified, and assuming superWIMPs from late decays make up all of the dark matter, with $\Omega_{\tilde{G}}=\Omega_{\mathrm{DM}}=0.23$, both the lifetime and energy release are determined by only two parameters: $m_{\tilde{G}}$ and $m_{\mathrm{NLSP}}$. The results for neutralino and slepton NLSPs are given in Fig. 13.

For the decay times of interest with redshifts $z \sim 10^{5}$ to $10^{7}$, the resulting photons interact efficiently through $\gamma e^{-} \rightarrow \gamma e^{-}$and $e X \rightarrow e X \gamma$, where $X$ is an ion, but photon number is conserved, since double Compton scattering $\gamma e^{-} \rightarrow \gamma \gamma e^{-}$is inefficient. The 
spectrum therefore relaxes to statistical but not thermodynamic equilibrium, resulting in a Bose-Einstein distribution function

$$
f_{\gamma}(E)=\frac{1}{e^{E /(k T)+\mu}-1}
$$

with chemical potential $\mu \neq 0$. Figure 13 also includes contours of $\mu$. The current bound is $\mu<9 \times 10^{-5}$ (159). Although there are at present no indications of deviations from black body, current limits are already sensitive to the superWIMP scenario, and future improvements may further probe superWIMP parameter space.

\section{BIG BANG NUCLEOSYNTHESIS}

Late time energy release after $t \sim 1$ s also destroys and creates light elements, potentially distorting the predictions of standard BBN. The impact depends sensitively on what the decaying NLSP is. For example, in the neutralino NLSP case, the neutralino decays generically through $\chi \rightarrow Z \tilde{G}, h \tilde{G}, \gamma \tilde{G}$. The first two modes lead to hadrons, which are very dangerous. Constraints from BBN on hadronic energy release essentially exclude the neutralino WIMP scenario, allowing only the fine-tuned case in which the neutralino is photino-like, $\chi \approx \tilde{\gamma}$, in which case the decays to $Z \tilde{G}$ and $h \tilde{G}$ are suppressed (160; 161). In the charged slepton NLSP case, the decaying WIMP may first bind with nuclei, which may enhance the effect of its decays on BBN (162; 163; 164; 165; 166).

BBN constraints on the gravitino superWIMP possibility have been considered in a number of studies. The results of one study, which considered minimal supergravity models with a stau NLSP decaying to a gravitino superWIMP, are given in Fig. 14. Without BBN constraints, we find that extremely large stau lifetimes are possible. The BBN constraints typically exclude the largest lifetimes (although there are interesting exceptions (167)). Nevertheless, lifetimes as large as $10^{4} \mathrm{~s}$ are still allowed for all stau masses, and even larger lifetimes are possible for light staus with mass $\sim 100 \mathrm{GeV}$. Models with large lifetimes and light staus are the most promising for the collider studies described in Sec. IV.D, and we see that BBN constraints do not exclude these scenarios.

Finally, late decays to superWIMPs may in fact improve the current disagreement of standard BBN predictions with the observed ${ }^{7} \mathrm{Li}$ and ${ }^{6} \mathrm{Li}$ abundances, although this typically requires that the decaying slepton be heavy, with mass above a $\mathrm{TeV}(168 ; 169 ; 170)$. For a review, see Ref. (171).

\section{SMALL SCALE STRUCTURE}

In contrast to WIMPs, superWIMPs are produced with large velocities at late times. This has two, a priori independent, effects. First, the velocity dispersion reduces the phase space density, smoothing out cusps in dark matter halos. Second, such particles damp the linear power spectrum, reducing power on small scales (173; 174; 175; 176; 177; 178; 179). As seen in Fig. 15, superWIMPs may suppress small scale structure as effectively as a $1 \mathrm{keV}$ sterile neutrino, the prototypical warm dark matter candidate (see Sec. VII). Some superWIMP scenarios may therefore be differentiated from standard cold dark matter scenarios by their impact on small scale structure; for a review, see Ref. (180). 


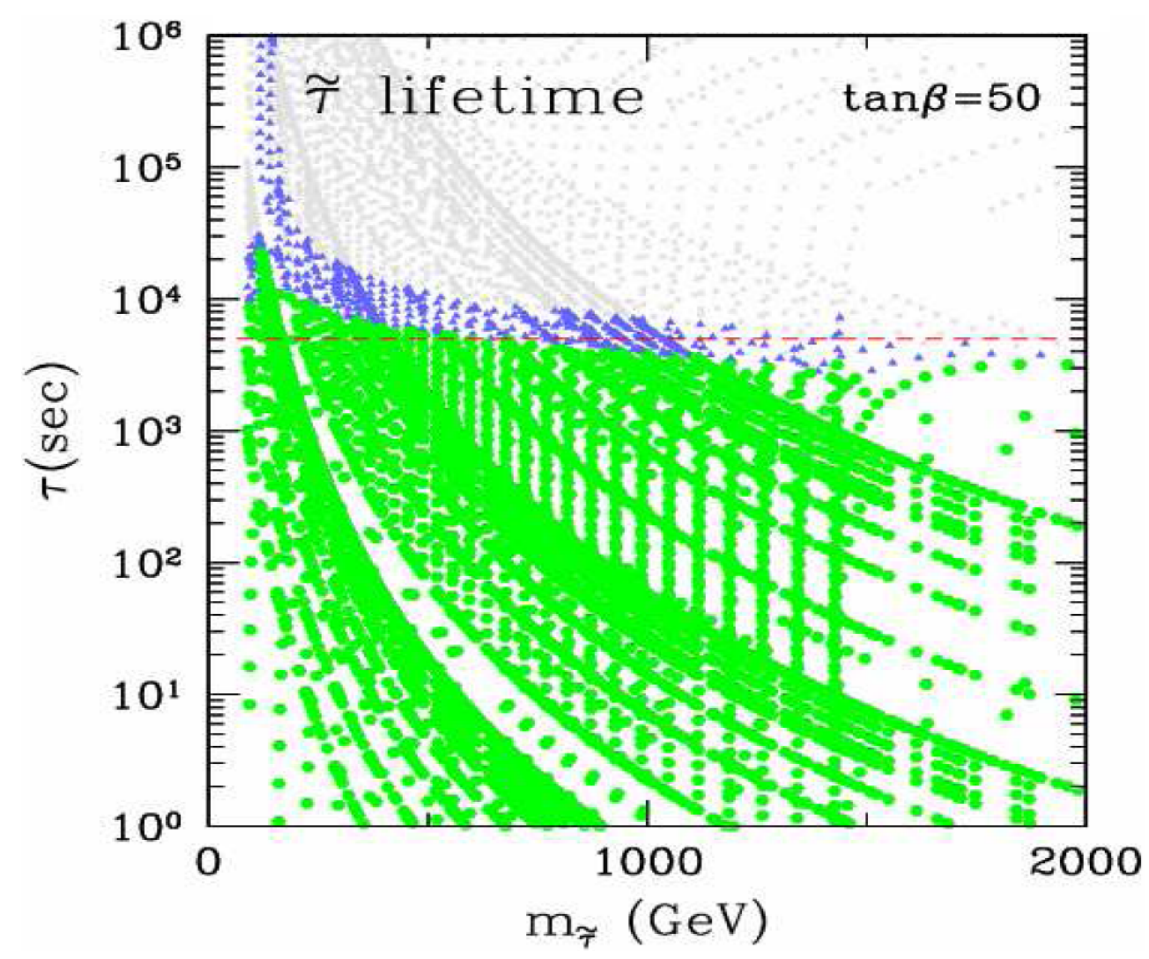

FIG. 14 Allowed values for the stau mass and lifetime in gravitino superWIMP scenarios scanning over minimal supergravity parameters with $\tan \beta=50$, and assuming gravitinos are produced both after reheating and in late decays. The grey dots represent models that satisfy collider constraints; the green dots represent models that also satisfy all BBN constraints; the blue dots represent models that are allowed for slightly loosened BBN constraints on ${ }^{6} \mathrm{Li} /{ }^{7} \mathrm{Li}$. From Ref. (172).

\section{LIGHT GRAVITINOS}

The gravitino dark matter candidates discussed in Sec. IV have masses around $m_{\text {weak }}$. Other well-motivated, if somewhat problematic, candidates are light gravitinos, with masses in the range $\mathrm{eV}-\mathrm{keV}$ and very different implications for experiments.

\section{A. Thermal Production}

\section{GAUGE-MEDIATED SUPERSYMMETRY BREAKING}

Low-energy supersymmetry elegantly addresses the gauge hierarchy problem but does not by itself solve the new physics flavor problem. In fact, the goal of solving the new physics flavor problem is the prime driver in the field of supersymmetric model building, and motivates a particularly elegant subset of supersymmetric theories known as gaugemediated supersymmetry breaking (GMSB) models (181; 182; 183; 184; 185; 186). In these models, supersymmetry-breaking is transmitted from a supersymmetry-breaking sector to the MSSM by so-called "messenger" particles through both gauge interactions and gravity. 


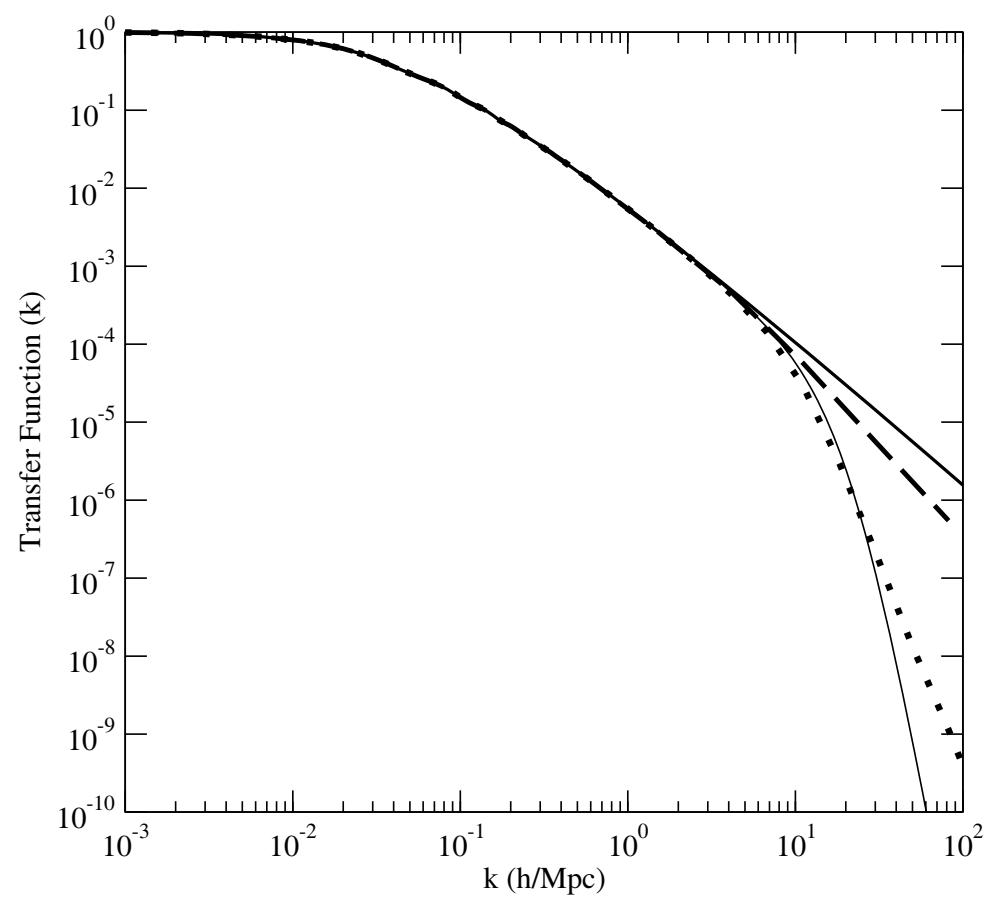

FIG. 15 The power spectrum for scenarios in which dark matter is completely composed of WIMPs (solid), half WIMPs and half superWIMPs (dashed), and completely composed of superWIMPs (dotted). For comparison, the lower solid curve is for $1 \mathrm{keV}$ sterile neutrino warm dark matter. From Ref. (176).

The resulting squark and slepton mass matrices, in $3 \times 3$ generation space, are of the form

$$
m_{\tilde{f}}^{2}=\left(\begin{array}{ccc}
m_{\mathrm{GMSB}}^{2} & 0 & 0 \\
0 & m_{\mathrm{GMSB}}^{2} & 0 \\
0 & 0 & m_{\mathrm{GMSB}}^{2}
\end{array}\right)+\left(\begin{array}{cc}
\sim m_{\mathrm{grav}}^{2} & \sim m_{\mathrm{grav}}^{2} \sim m_{\mathrm{grav}}^{2} \\
\sim m_{\mathrm{grav}}^{2} & \sim m_{\mathrm{grav}}^{2} \sim m_{\mathrm{grav}}^{2} \\
\sim m_{\mathrm{grav}}^{2} & \sim m_{\mathrm{grav}}^{2} \sim m_{\mathrm{grav}}^{2}
\end{array}\right)
$$

where

$$
m_{\mathrm{GMSB}} \sim \frac{g^{2}}{16 \pi^{2}} \frac{F}{M_{\mathrm{m}}} \quad \text { and } \quad m_{\text {grav }} \sim \frac{F}{M_{*}} .
$$

The parameter $g$ denotes gauge coupling constants, $F$ is the supersymmetry-breaking scale squared, $M_{*}$ is the reduced Planck mass, and $M_{\mathrm{m}}$ is another mass scale determined by the supersymmetry-breaking sector and is related to the mass of the messenger particles.

The essential feature is that the GMSB contributions are determined by gauge coupling constants only and so are generation-blind. They therefore do not mediate flavor-changing effects, and if they are dominant, such theories ameliorate the new physics flavor problem. For them to be dominant, one assumes $M_{\mathrm{m}} \ll M_{*}$ and $F \ll\left(10^{11} \mathrm{GeV}\right)^{2}$ subject to the constraint $F / M_{\mathrm{m}} \sim 100 \mathrm{TeV}$, so that the superpartner masses are at the weak scale, but the flavor-violating gravity contributions are negligible.

As seen in Eq. (20), however, $m_{\text {grav }} \sim m_{\tilde{G}}$, that is, the gravity contributions to the squark and sleptons masses are the same size as the gravitino mass. In GMSB scenarios, then, the LSP is the gravitino. To sufficiently suppress flavor-violating effects, one typically requires $m_{\text {grav }}, m_{\tilde{G}} \lesssim 1 \mathrm{GeV}$. As a result, WIMPs and superWIMPs are typically not viable dark matter candidates in GMSB models: WIMPs decay through $R$-parity conserving 
interactions such as $\chi \rightarrow \gamma \tilde{G}$, and superWIMPs are under-abundant, since $m_{\tilde{G}} \ll m_{\text {WIMP }}$ implies $\Omega_{\text {SWIMP }} \ll \Omega_{\text {WIMP }}$.

\section{RELIC DENSITY}

Light gravitinos may be dark matter candidates in GMSB models, however (187). As with neutrinos, light gravitinos may be in thermal equilibrium in the early hot Universe. They then decouple, with relic density

$$
\Omega_{\tilde{G}}^{\mathrm{th}} \approx 0.25 \frac{m_{\tilde{G}}}{100 \mathrm{eV}} .
$$

When originally proposed, $\Omega_{\mathrm{DM}} \sim 1$ was possible, and constraints from structure formation allowed $m_{\tilde{G}} \sim 100 \mathrm{eV}-\mathrm{keV}$. Such "keV gravitinos" were then viable dark matter candidates. There is no theoretical reason to favor the $100 \mathrm{eV}-\mathrm{keV}$ mass range for gravitinos, and so this scenario does not naturally explain the relic density in the way that the WIMP miracle does. In contrast to WIMPs and superWIMPs, however, light gravitinos are well-motivated by their presence in models that solve not only the gauge hierarchy problem, but also the new physics flavor problem.

The light gravitino scenario has become much less simple in recent years, however. The allowed value for the dark matter relic density has been reduced to $\Omega_{\mathrm{DM}} \simeq 0.23$. In addition, there are much more stringent limits on how hot dark matter can be. Among the strongest bounds are those from Lyman- $\alpha$ forest observations, which constrain the distribution of gas between distant objects and us. Observations of density fluctuations on relatively small scales implies that dark matter should not have erased power on these scales. Lyman- $\alpha$ constraints therefore require that the bulk of dark matter be sufficiently cold, implying $m_{\tilde{G}} \gtrsim 2 \mathrm{keV}$ (188; 189). Together, these developments have closed the window on the minimal light gravitino dark matter scenario.

There are, however, at least two viable extensions. In the one-component gravitino scenario, sometimes abbreviated $\Lambda \mathrm{WDM}$, the gravitino has mass $\gtrsim 2 \mathrm{keV}$ and is cold enough to agree with Lyman- $\alpha$ constraints, but its thermal relic density is either not realized, for example, because of a low reheating temperature, or is significantly diluted, for example, through late entropy production (190). Alternatively, in the two-component gravitino scenario, typically denoted $\Lambda$ CWDM, the gravitino has mass $\lesssim 16 \mathrm{eV}(\underline{191})$, but it is a sufficiently small portion of the dark matter to be consistent with structure formation constraints, provided another particle provides an additional and dominant cold or warm component.

\section{B. Particle Colliders}

Remarkably, the $\Lambda \mathrm{WDM}$ and $\Lambda \mathrm{CWDM}$ scenarios may be differentiated at particle colliders. In contrast to weak-scale gravitinos, light gravitinos interactions are stronger than gravitational, as they are enhanced by their spin 1/2 longitudinal Goldstino components. These interactions depend on the supersymmetry-breaking scale $F$ and are stronger for lighter gravitinos. For example, a Bino-like neutralino has decay width

$$
\Gamma(\tilde{B} \rightarrow \gamma \tilde{G})=\frac{\cos ^{2} \theta_{W} m_{\tilde{B}}^{5}}{16 \pi F^{2}}
$$


where $\theta_{W}$ is the weak mixing angle, corresponding to a decay length

$$
c \tau \simeq 22 \mathrm{~cm}\left[\frac{m_{\tilde{G}}}{100 \mathrm{eV}}\right]^{2}\left[\frac{100 \mathrm{GeV}}{m_{\tilde{B}}^{2}}\right]^{5} .
$$

The two light gravitino scenarios therefore predict decay lengths that are either shorter or longer than typical sizes of particle detectors. In a Bino LSP GMSB scenario, supersymmetry events will be seen through missing energy in the $\Lambda$ WDM scenario, but will be characterized by two high energy photons in the $\Lambda$ CWDM scenario. Such diphoton events are the subject of ongoing searches at the Tevatron (192) and will be spectacular at the LHC. Similar results hold for the stau NLSP scenario and the decay $\tilde{\tau} \rightarrow \tau \tilde{G}$ : supersymmetry events will appear with pairs of metastable heavy charged particles in the $\Lambda$ WDM scenario, but will be characterized by two high energy taus in the $\Lambda$ CWDM scenario. The observation of these various event types, when interpreted in the gravitino dark matter framework, may therefore have strong cosmological implications, for example, indicating an era of late entropy production in the $\Lambda \mathrm{WDM}$ case, or strongly implying the existence of another, dominant, form of dark matter in the $\Lambda$ CWDM scenario.

\section{HIDDEN DARK MATTER}

As noted in Sec. II, despite great recent progress, all solid evidence for dark matter is gravitational. There is also strong evidence against dark matter having strong or electromagnetic interactions. A logical possibility, then, is hidden dark matter, that is, dark matter that has no SM gauge interactions. Hidden dark matter has been explored for decades and brings with it a great deal of model building freedom, leading to a large and diverse class of candidates (193; 194; 195; 196; 197). Unfortunately, this freedom comes at a cost: by considering hidden dark matter, one typically loses (1) connections to the central problems of particle physics discussed in Sec. II.B, (2) the WIMP miracle, and (3) predictivity, since most hidden dark matter candidates have no non-gravitational signals, which are most likely required if we are to identify dark matter.

Recently, however, some hidden dark matter models have been shown to have some, or even all, of the three properties listed above, putting them on more solid footing and providing extra structure and motivation for this framework. In this section, we first consider the general possibility of hidden sectors, but then focus on this subset of hidden dark matter candidates and explore their properties and implications for detection.

\section{A. Thermal Freeze Out}

\section{CONSTRAINTS ON TEMPERATURE AND DEGREES OF FREEDOM}

The thermal history of hidden sectors may differ from that of the visible sector. However, the hidden sector's temperature, along with its "size," is constrained, to the extent that it affects the cosmological history of the visible sector.

One of the leading constraints on hidden sectors is provided by BBN. The success of BBN is highly sensitive to the expansion rate of the Universe at time $t_{\mathrm{BBN}} \sim 1-1000 \mathrm{~s}$. Light degrees of freedom in a hidden sector change the expansion rate of the Universe and thereby impact BBN, even if they have no SM interactions. The constraint is conventionally quoted 
as a bound on $n_{\text {eff }}$, the effective number of light neutrino species, and may be taken to be $n_{\mathrm{eff}}=3.24 \pm 1.2(95 \% \mathrm{CL})(198 ; 199 ;$; 200) $)$. This implies

$$
g_{*}^{h}\left(T_{\mathrm{BBN}}^{h}\right)\left(\frac{T_{\mathrm{BBN}}^{h}}{T_{\mathrm{BBN}}}\right)^{4}=\frac{7}{8} \cdot 2 \cdot\left(n_{\mathrm{eff}}-3\right) \leq 2.52(95 \% \mathrm{CL}),
$$

where $T_{\mathrm{BBN}}^{h}$ and $T_{\mathrm{BBN}}$ are the temperatures of the hidden and visible sectors at time $t_{\mathrm{BBN}}$. This is a significant constraint (201); for example, Eq. (32) excludes a hidden sector that is an exact copy of the SM with $g_{*}^{h}\left(T_{\mathrm{BBN}}^{h}\right)=10.75$, assuming it has the same temperature as the visible sector, so $T_{\mathrm{BBN}}^{h}=T_{\mathrm{BBN}}$.

As evident in Eq. (32), however, this statement is highly sensitive to the hidden sector's temperature. If the observable and hidden sectors are not in thermal contact, the hidden sector may be colder than the observable sector. This would be the case if, for example, the inflaton couplings to the observable and hidden sectors are not identical, so that they reheat to different temperatures (196; 197). Alternatively, the observable and hidden sectors may initially have the same temperature, either because they have the same inflaton couplings or because they are in thermal contact, but may cool independently and have different temperatures at later times. For hidden sector temperatures that are now half of the observable sector's temperature, hundreds of degrees of freedom, equivalent to several copies of the SM or MSSM, may be accommodated.

\section{THE WIMPLESS MIRACLE}

It is desirable for hidden dark matter to have naturally the correct relic density, just as in the case of WIMPs. One way to achieve this goal would be to duplicate the couplings and mass scales of the visible sector in the hidden sector so that the WIMP miracle is satisfied in the hidden sector. Given the discussion above, this is certainly possible if the hidden sector has fewer light degrees of freedom than the visible sector or is slightly colder.

At the same time, this possibility is both rather unmotivated and far too rigid. As discussed in Sec. III.A.1, the thermal relic density of a stable particle with mass $m_{X}$ annihilating through interactions with couplings $g_{X}$ is

$$
\Omega_{X} \sim\left\langle\sigma_{A} v\right\rangle^{-1} \sim \frac{m_{X}^{2}}{g_{X}^{4}} .
$$

The WIMP miracle is the fact that, for $m_{X} \sim m_{\text {weak }}$ and $g_{X} \sim g_{\text {weak }} \simeq 0.65, \Omega_{X}$ is roughly $\Omega_{\mathrm{DM}} \approx 0.23$.

Equation (33) makes clear, however, that the thermal relic density fixes only one combination of the dark matter's mass and coupling, and other combinations of $\left(m_{X}, g_{X}\right)$ can also give the correct $\Omega_{X}$. In the SM, $g_{X} \sim g_{\text {weak }}$ is the only choice available, but in a general hidden sector, with its own matter content and gauge forces, other values of $\left(m_{X}, g_{X}\right)$ may be realized. Such models generalize the WIMP miracle to the "WIMPless miracle": dark matter that naturally has the correct relic density, but does not necessarily have a weak-scale mass or weak interactions (202).

\section{B. Candidates}

The WIMPless miracle is naturally realized in particle physics frameworks that have several other motivations. As an example, consider the supersymmetric models with GMSB 


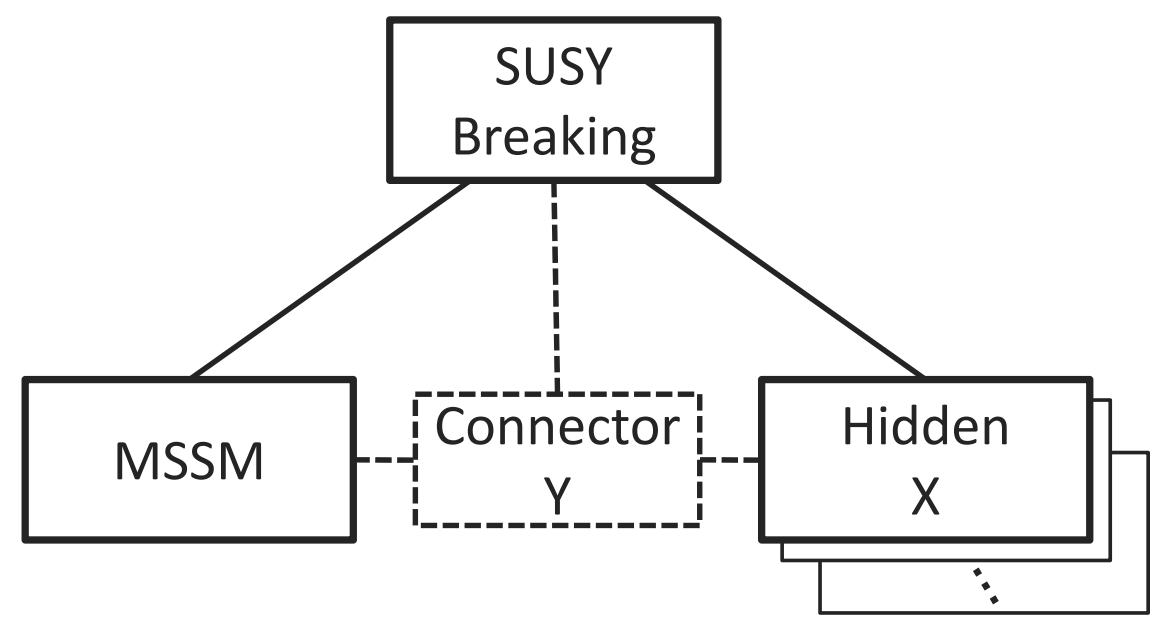

FIG. 16 Sectors of supersymmetric models. Supersymmetry breaking is mediated by gauge interactions to the MSSM and the hidden sector, which contains the dark matter particle $X$. An optional connector sector contains fields $Y$, charged under both MSSM and hidden sector gauge groups, which induce signals in direct and indirect searches and at colliders. There may also be other hidden sectors, leading to multi-component dark matter. From Ref. (202).

described previously in Sec. V.A.1. These models necessarily have several sectors, as shown in Fig. 16. The supersymmetry-breaking sector includes the fields that break supersymmetry dynamically and the messenger particles that mediate this breaking to other sectors through gauge interactions. The MSSM sector includes the fields of supersymmetric extension of the SM. In addition, supersymmetry breaking may be mediated to one or more hidden sectors. The hidden sectors are not strictly necessary, but there is no reason to prevent them, and hidden sectors are ubiquitous in such models originating in string theory.

As described in Sec. V.A.1, the essential feature of GMSB models is that they elegantly address the new physics flavor problem by introducing generation-independent squark and slepton masses of the form

$$
m \sim \frac{g^{2}}{16 \pi^{2}} \frac{F}{M_{\mathrm{m}}} .
$$

The generic feature is that superpartner masses are proportional to gauge couplings squared times the ratio $F / M_{\mathrm{m}}$, which is a property of the supersymmetry-breaking sector. With analogous couplings of the hidden sector fields to hidden messengers, the hidden sector superpartner masses are

$$
m_{X} \sim \frac{g_{X}^{2}}{16 \pi^{2}} \frac{F}{M_{\mathrm{m}}}
$$

where $g_{X}$ is the relevant hidden sector gauge coupling. As a result,

$$
\frac{m_{X}}{g_{X}^{2}} \sim \frac{m}{g^{2}} \sim \frac{F}{16 \pi^{2} M_{\mathrm{m}}}
$$

that is, $m_{X} / g_{X}^{2}$ is determined solely by the supersymmetry-breaking sector. As this is exactly the combination of parameters that determines the thermal relic density of Eq. (33), the hidden sector automatically includes a dark matter candidate that has the desired thermal relic density, irrespective of its mass. This has been verified numerically for a concrete 


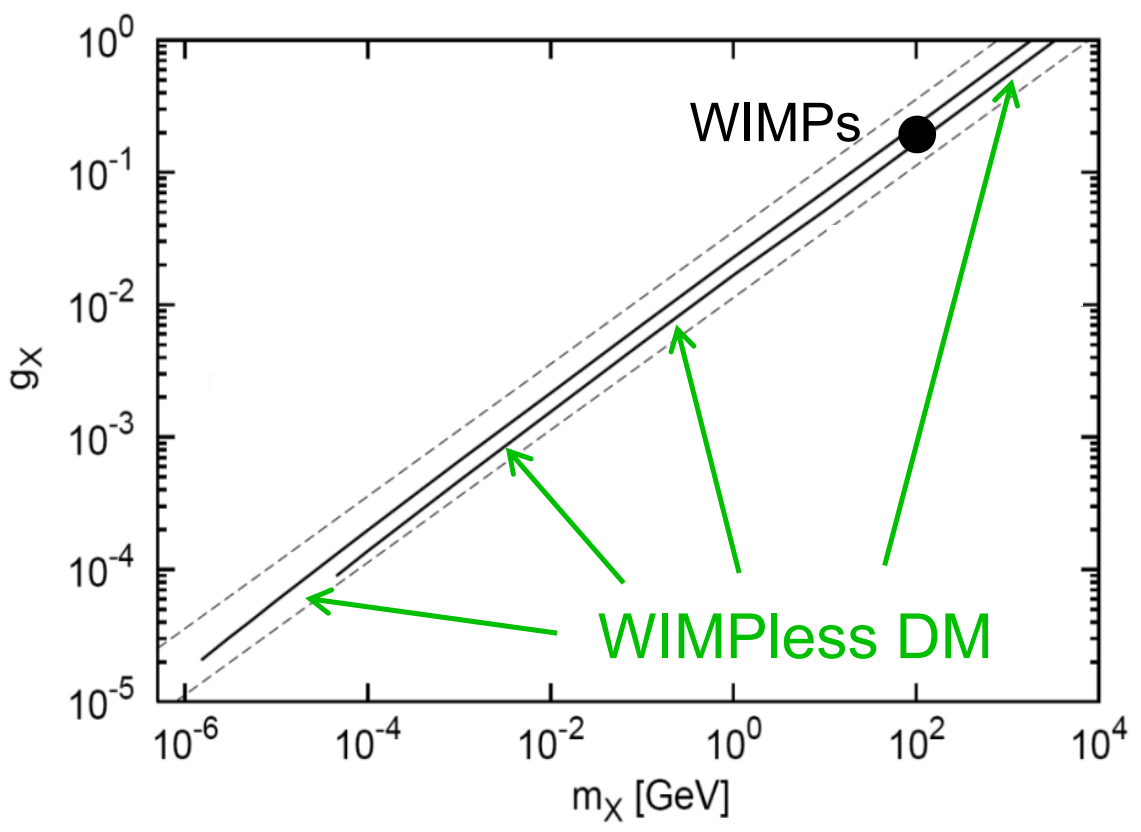

FIG. 17 Contours of $\Omega_{X} h^{2}=0.11$ in the $\left(m_{X}, g_{X}\right)$ plane for hidden to observable reheating temperature ratios $T_{\mathrm{RH}}^{h} / T_{\mathrm{RH}}=0.8$ (upper solid) and 0.3 (lower solid), where the hidden sector is a 1-generation flavor-free version of the MSSM. Also plotted are lines of $m_{\text {weak }} \equiv\left(m_{X} / g_{X}^{2}\right) g^{\prime 2}=$ $100 \mathrm{GeV}$ (upper dashed) and $1 \mathrm{TeV}$ (lower dashed). The WIMPless hidden models generalize the WIMP miracle to a family of models with other dark matter masses and couplings. From Ref. (204).

hidden sector model; the results are shown in Fig. 17. This property relies on the relation $m_{X} \propto g_{X}^{2}$, which is common to other frameworks for new physics that avoid flavor-changing problems, such as anomaly-mediated supersymmetry breaking. The "coincidence" required for WIMPless dark matter may also be found in other settings; see, e.g., Ref. (203).

WIMPless and other hidden sector models also naturally open the possibility of dark forces in the hidden sector. In the WIMPless scenarios just described, this possibility arises naturally if one attempts to understand why the hidden sector particle is stable. This is an important question; after all, in these GMSB models, all SM superpartners decay to the gravitino. In the hidden sector, an elegant way to stabilize the dark matter is through U(1) charge conservation. This possibility necessarily implies massless gauge bosons in the hidden sector. Alternatively, the hidden sector may have light, but not massless, force carriers, as described in Sec. III.D. In all of these cases, the dynamics of the hidden sector may have many interesting astrophysical implications, some of which are discussed in Sec. VI.F.

In summary, hidden sector dark matter models may in fact be motivated by leading problems in particle physics, and may even have naturally the correct relic density, through a generalization of the WIMP miracle to the WIMPless miracle. In fact, the third virtue discussed in Sec. VI, predictivity, may also be restored, and we now describe non-gravitational signals of hidden dark matter.

\section{Direct Detection}

The decoupling of the WIMP miracle from WIMPs has many possible implications and observable consequences. In the case that the dark matter is truly hidden, it implies that 
there are no prospects for direct or indirect detection. Signals must be found in astrophysical observations, as in the case of superWIMPs. Alternatively, there may be connector sectors containing particles that mediate interactions between the SM and the hidden sector through non-gauge (Yukawa) interactions (see Fig. 16). Such connectors may generate many signals with energies and rates typically unavailable to WIMPs.

As an example, first consider direct detection. As discussed in Sec. III.C, the DAMA and CoGeNT signals may be explained without violating other bounds if the dark matter mass and spin-independent cross section are in the ranges $\left(m_{X}, \sigma_{\mathrm{SI}}\right) \sim\left(1-10 \mathrm{GeV}, 10^{-41}-\right.$ $\left.10^{-39} \mathrm{~cm}^{2}\right)$. Such masses are low for conventional WIMPs, and the cross section is also very high. In WIMPless models, however, where the thermal relic density is achieved for a variety of dark matter masses, such masses are perfectly natural. Furthermore, a WIMPless particle $X$ may couple to the SM through Yukawa interactions

$$
\mathcal{L}=\lambda_{f} X \bar{Y}_{L} f_{L}+\lambda_{f} X \bar{Y}_{R} f_{R}
$$

where $Y$ is a vector-like connector fermion, and $f$ is a SM fermion. Taking $f$ to be the $b$ quark, and the $Y$ mass to be $400 \mathrm{GeV}$, consistent with current bounds, these couplings generate spin-independent scattering cross sections given in Fig. 18. We see that WIMPless dark matter may explain the DAMA and CoGeNT results. Other proposed hidden dark matter explanations of DAMA and CoGeNT include those of Ref. (205; 206).

\section{Indirect Detection}

WIMPless dark matter also provides new target signals for indirect detection. For WIMPs, annihilation cross sections determine both the thermal relic density and indirect detection signals. The thermal relic density therefore constrains the rates of indirect detection signals. In the WIMPless case, however, this connection is weakened, since the thermal relic density is governed by hidden sector annihilation and gauge interactions, while the indirect detection signals are governed by the interactions of Eq. (37).

This provides a wealth of new opportunities for indirect detection experiments looking for photons, positrons, and other annihilation products. As an example, WIMPless dark matter may be detected through its annihilation to neutrinos in the Sun by experiments such as Super-Kamiokande. Although such rates depend on the competing cross sections for capture and annihilation, the Sun has almost certainly reached its equilibrium state, and the annihilation rate is determined by the scattering cross section (73). The prospects for Super-Kamiokande may therefore be compared to direct detection rates (73; 87; 88; 89). The results are given in Fig. 18. In the near future, Super-Kamiokande and other neutrino detectors may be able to probe the low mass regions corresponding to the DAMA and CoGeNT signals.

\section{E. Particle Colliders}

If hidden dark matter does not interact with SM particles, there are no collider signals. However, there may be connector particles. Since these necessarily have SM interactions, they may be produced with large cross sections at colliders, and since they necessarily have hidden charge, their decays may be non-standard, leading to unusual signatures. 


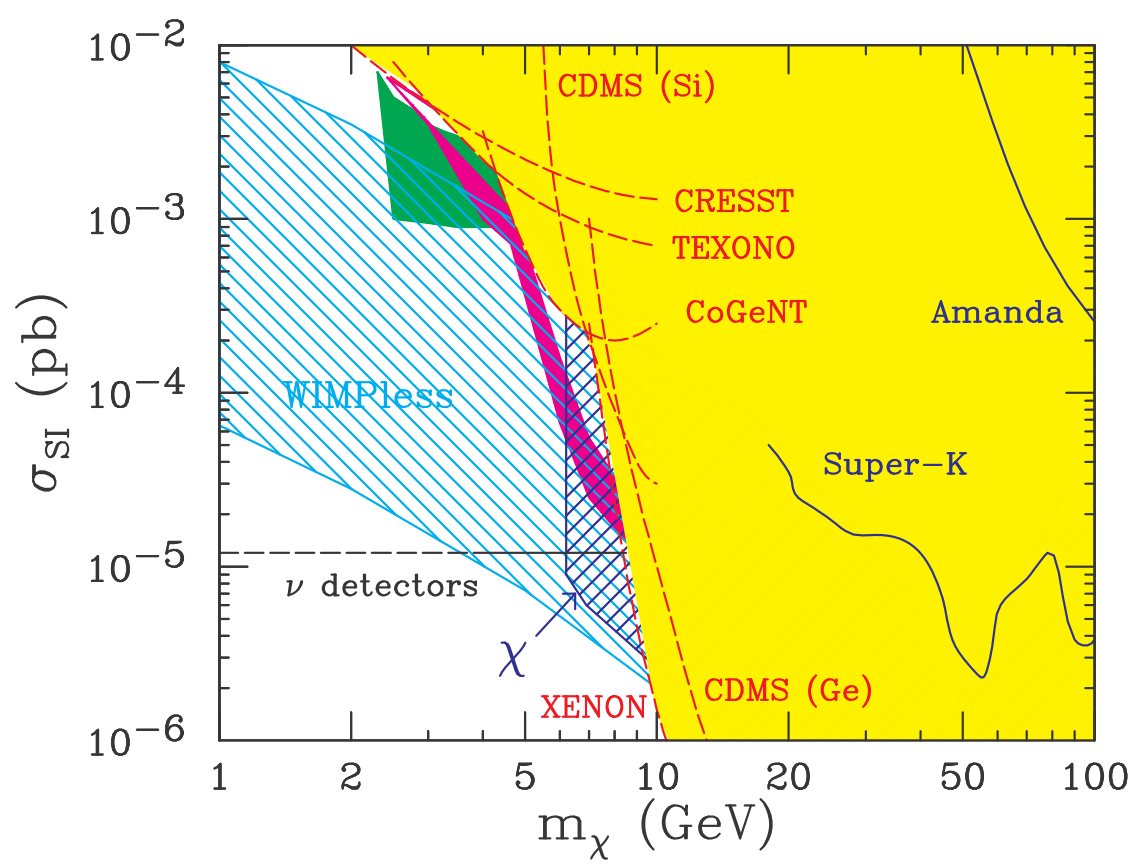

FIG. 18 Direct detection cross sections for spin-independent $X$-nucleon scattering in the low dark matter mass $m_{X}$ region. The large hatched region is the predictions of WIMPless models with connector mass $m_{Y}=400 \mathrm{GeV}$ and $0.3<\lambda_{b}<1.0$. The small hatched region is the prediction of neutralino models considered in Ref. (207). The magenta shaded region is DAMA-favored given channeling and no streams (208), and the medium green shaded region is DAMA-favored at $3 \sigma$ given streams and no channeling (81). The light yellow shaded region is excluded by the direct detection experiments CRESST (209), CDMS (210), XENON10 (61), TEXONO (211), and CoGeNT (212), as indicated. The blue contours are the published Super-Kamiokande (73) and AMANDA (75) exclusion limits, and the black line is a projection of future neutrino detector sensitivity. From Ref. (89).

As an example, consider the connector sector interactions specified in Eq. (37). In the hadronic version with $f=q$, the $Y$ necessarily have strong interactions, and so will be similar to 4th generation quarks; for $f=\ell$, the $Y$ particles are similar to 4th generation leptons. The existence of 4 th generation quarks and leptons is constrained by direct collider searches and also by precision electroweak measurements from LEP and the SLC, but is far from excluded (213). 4th generation quarks with masses in the range $300 \mathrm{GeV} \lesssim m_{T^{\prime}, B^{\prime}} \lesssim$ $600 \mathrm{GeV}$ are consistent with all data. Strongly-interacting connector particles with mass in this range will be produced in large numbers at the LHC, and also at the Tevatron. At the same time, such connector particles are unlike standard 4th generation quarks, which dominantly decay through charged-current modes, such as $t^{\prime} \rightarrow W b$ and $b^{\prime} \rightarrow W t$. Connector particles will decay through $T^{\prime} \rightarrow t X$, and $B^{\prime} \rightarrow b X$, similar to squarks, except that squarks typically decay through cascades, which produce leptons. Hidden sector dark matter therefore provides new motivation for relatively simple signatures with missing energy carried away by the hidden dark matter (214). Hidden sectors and connectors may also impact the properties of SM particles, with consequences for colliders running below the energy frontier (215).

Hidden sector gauge forces may also have other observable effects. For example, hid- 
den photons may mix with SM photons through kinetic mixing terms $F^{\mu \nu} F_{\mu \nu}^{h}$, leading to fractionally charged particles (216) that are subject to a wide variety of probes $(217 ; 218)$.

\section{F. Astrophysical Signals}

As explained in Sec. VI.B, an elegant way to stabilize a hidden sector dark matter candidate is through hidden charge conservation, much like the electron is stabilized by $\mathrm{U}(1)$ charge conservation in the SM. This requires that the dark matter have hidden charge and interact through hidden photons or other gauge bosons. More generally, hidden dark matter may interact through massive, but light, force carriers. In both cases, the hidden dark matter has significant self-interactions. Velocity-independent ("hard sphere") self-interactions have been extensively studied, for example, in the strongly self-interacting framework of Ref. (219). Recent work on hidden dark matter has motivated new interest in velocity-dependent cross sections, namely, the classic Coulomb scattering cross section

$$
\frac{d \sigma}{d \Omega}=\frac{\alpha_{X}^{2}}{m_{X}^{2} v^{4} \sin ^{4} \frac{\theta}{2}}
$$

and its generalization to Yukawa scattering for massive gauge bosons.

Charged hidden dark matter has many astrophysical implications: (1) bound state formation and Sommerfeld-enhanced annihilation after chemical freeze out may reduce its relic density (111; 112); (2) similar effects greatly enhance dark matter annihilation in protohalos at redshifts of $z \sim 30$ (110); (3) Compton scattering off hidden photons delays kinetic decoupling, suppressing small scale structure (220); and (4) Rutherford scattering makes such dark matter collisional (220; 221).

The last possibility, of collisional dark matter, may lead to a number of observable effects. A well-known probe of dark matter self-interactions is provided by the Bullet Cluster, a rare system where a subcluster is seen to be moving through a larger cluster with relative velocity $\sim 4500 \mathrm{~km} / \mathrm{s}(9)$. Through observations in the optical and X-ray and strong and weak gravitational lensing observations, it is clear that dark matter tracks the behavior of stars, which are collisionless, rather than the gas. These observations have allowed stringent bounds to be placed on the dark matter self-interaction strength. For velocity-independent cross sections, the Bullet Cluster observations imply $\sigma_{\mathrm{DM}} / m_{X} \lesssim 3000 \mathrm{GeV}^{-3}\left(\sigma_{\mathrm{DM}} / m_{X} \lesssim\right.$ $\left.0.7 \mathrm{~cm}^{2} / \mathrm{g}\right)$. These are the most direct constraints on the self-interaction of dark matter. They have been adapted to the case of velocity-dependent cross sections, leading to the bounds on dark matter mass $m_{X}$ and coupling strength $\alpha_{X}$ shown in Fig. 19 .

Self-interactions also allow dark matter particles to transfer energy. Self-interactions that are strong enough to create $\mathcal{O}(1)$ changes in the velocities of dark matter particles will isotropize the velocity dispersion and create spherical halos. These expectations are borne out by simulations of self-interacting dark matter in the hard sphere limit (219, 222; 223). The existence of elliptical dark matter halos may therefore also constrain self-interactions (224). The average rate for dark matter particles to change velocities by $\mathcal{O}(1)$ factors is

$$
\Gamma_{k}=\int d^{3} v_{1} d^{3} v_{2} f\left(v_{1}\right) f\left(v_{2}\right)\left(n_{X} v_{\text {rel }} \sigma_{T}\right)\left(v_{\text {rel }}^{2} / v_{0}^{2}\right),
$$

where $f(v)=e^{-v^{2} / v_{0}^{2}} /\left(v_{0} \sqrt{\pi}\right)^{3}$ is the dark matter's assumed (Maxwellian) velocity distribution, $n_{X}$ is its number density, $v_{\text {rel }}=\left|\vec{v}_{1}-\vec{v}_{2}\right|$, and $\sigma_{T}=\int d \Omega_{*}\left(d \sigma / d \Omega_{*}\right)\left(1-\cos \theta_{*}\right)$ is the 


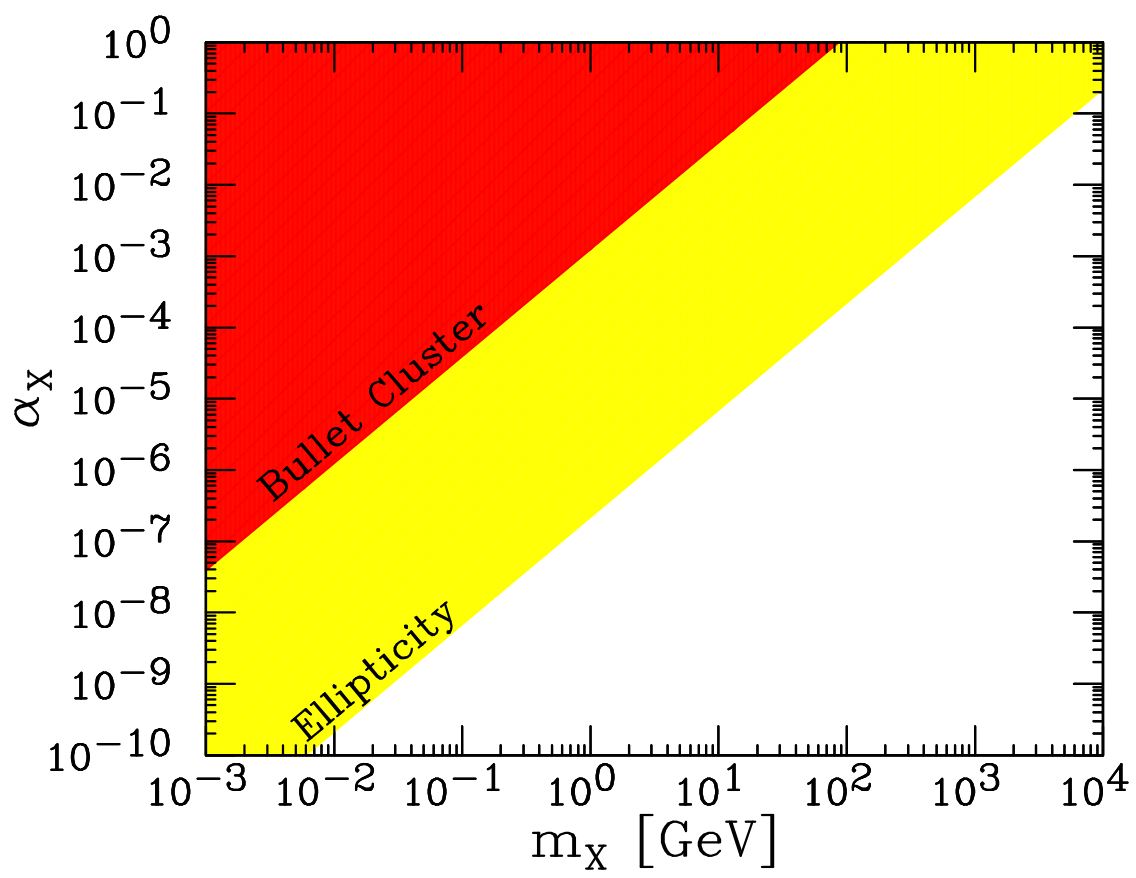

FIG. 19 Allowed regions in $\left(m_{X}, \alpha_{X}\right)$ plane, where $m_{X}$ is the mass of dark matter charged under hidden electromagnetism with fine-structure constant $\alpha_{X}$. The shaded regions are excluded by constraints from Bullet Cluster bounds on self-interactions (dark red) and the observed ellipticity of galactic dark matter halos (light yellow). From Ref. (220).

energy transfer cross section, where $\theta_{*}$ is the scattering angle in the center-of-mass frame. X-ray observations have established the ellipticity of the dark matter halo of the elliptical galaxy NGC 720 (225; 226), and requiring $\Gamma_{k}^{-1}>10^{10}$ Gyr for this system also constrains self-interacting hidden dark matter, as shown in Fig. 19. Note that these constraints are much stronger than those from the Bullet Cluster: for elliptical halos with dark matter velocities $v \sim 10^{-3}$, the cross section is greatly enhanced relative to the Bullet Cluster with its larger velocities.

The possibility that dark matter is stabilized by hidden charge conservation also motivates other astrophysical signals, such as time delays of light passing through dark matter; see, e.g., Ref. (227).

\section{STERILE NEUTRINOS}

The evidence for neutrino mass described in Sec. II.B.3 requires new physics beyond the SM. This problem may be resolved by adding right-handed neutrinos $\nu^{\alpha} \equiv \nu_{R}^{\alpha}$, so that neutrinos may get mass through the same mechanism that generates masses for the quarks and charged leptons. For the mass terms to be allowed under the symmetries of the SM, the right-handed neutrinos must have no SM gauge interactions. One may therefore also add a gauge-invariant term to the Lagrangian involving only two right-handed neutrinos - the so-called Majorana mass term.

The SM is therefore extended to include $N$ sterile neutrinos by adding the terms

$$
\mathcal{L}_{\nu_{R}}=\bar{\nu}^{\alpha} i \not D \nu^{\alpha}-\left(\lambda_{i \beta}^{\nu} \bar{L}^{i} \nu^{\beta} \tilde{\phi}+\text { h.c. }\right)-\frac{1}{2} M_{\alpha \beta} \bar{\nu}^{\alpha} \nu^{\beta},
$$


where $\lambda_{i \beta}^{\nu}$ are the neutrino Yukawa couplings, $M_{\alpha \beta}$ is the Majorana mass matrix, and $\alpha, \beta=$ $1, \ldots, N$, where $N \geq 2$ so that at least two neutrino states are massive. When electroweak symmetry is broken, the Higgs field gets a vacuum expectation value. The neutrino mass eigenstates are then determined by diagonalizing the complete $(3+N) \times(3+N)$ neutrino mass matrix

$$
m_{\nu}=\left(\begin{array}{cc}
0 & \lambda_{i \beta}\langle\phi\rangle \\
\lambda_{\alpha j}^{*}\langle\phi\rangle & M_{\alpha \beta}
\end{array}\right) .
$$

Mass eigenstates that are dominantly linear combinations of left-handed neutrinos are called "active" neutrinos, and those that are dominated by right-handed neutrino components are called "sterile" neutrinos.

An elegant idea for explaining the very small neutrino masses is the see-saw mechanism. In this framework, one assumes $\lambda^{\nu} \sim \mathcal{O}(1)$ and $M \gg\langle\phi\rangle$. There are then $N$ large neutrino masses $\sim M$, and three small neutrino masses $\sim \lambda^{\nu 2} / M$. For $M \sim 10^{14} \mathrm{GeV}$, near the grand unification scale, one gets the desired light neutrino masses. In this case, sterile neutrinos are beyond the range of experiments and are not dark matter candidates.

On the other hand, given the range of fermion masses illustrated in Fig. 1, there is clearly a range of Yukawa couplings in the SM and there is no strong reason to assume $\lambda^{\nu} \sim \mathcal{O}(1)$. In general, one may, then, have light sterile neutrinos, which may be dark matter candidates. We denote this neutrino $\nu_{s}$, with mass $m_{s}$ and mixing angle $\theta$ defined by

$$
\nu_{s}=\cos \theta \nu_{R}+\sin \theta \nu_{L}
$$

where $\nu_{R}\left(\nu_{L}\right)$ is a linear combination of right-handed (left-handed) gauge eigenstates.

\section{A. Production Mechanisms}

Sterile neutrinos may be produced in a number of ways. The relic density depends on the sterile neutrino mass and mixing angle, and all of the mechanisms require very small masses and mixing angles for sterile neutrinos to be viable dark matter candidates. These parameters are not independently motivated by other theoretical arguments, and so sterile neutrinos do not naturally have the right relic density. At the same time, sterile neutrinos with these masses and mixings may explain some observations, described in Secs.VII.B and VII.C, which may be taken as observational evidence for these parameters.

Sterile neutrinos may be produced by oscillations at temperatures $T \sim 100 \mathrm{MeV}(228)$. Sterile neutrinos were never in thermal equilibrium, but the resulting distribution is near thermal. The results of numerical studies may be approximated by

$$
\Omega_{\nu_{s}} \approx 0.2 \frac{\sin ^{2} 2 \theta}{10^{-8}}\left[\frac{m_{s}}{3 \mathrm{keV}}\right]^{1.8}
$$

this is shown in Fig. 20 as the $L=0$ contour. This production mechanism is always present, and the region to the right of this contour is excluded by overclosure.

The sterile neutrino relic abundance may be enhanced if the Universe has a non-zero lepton number asymmetry

$$
L=\frac{n_{\nu_{a}}-n_{\bar{\nu}_{a}}}{n_{\gamma}}
$$

For values of $L \gtrsim 10^{-3}$ that are nevertheless small enough to be well within current limits from $\mathrm{BBN}$, this can allow sterile neutrinos to be all of the dark matter for smaller mixing 


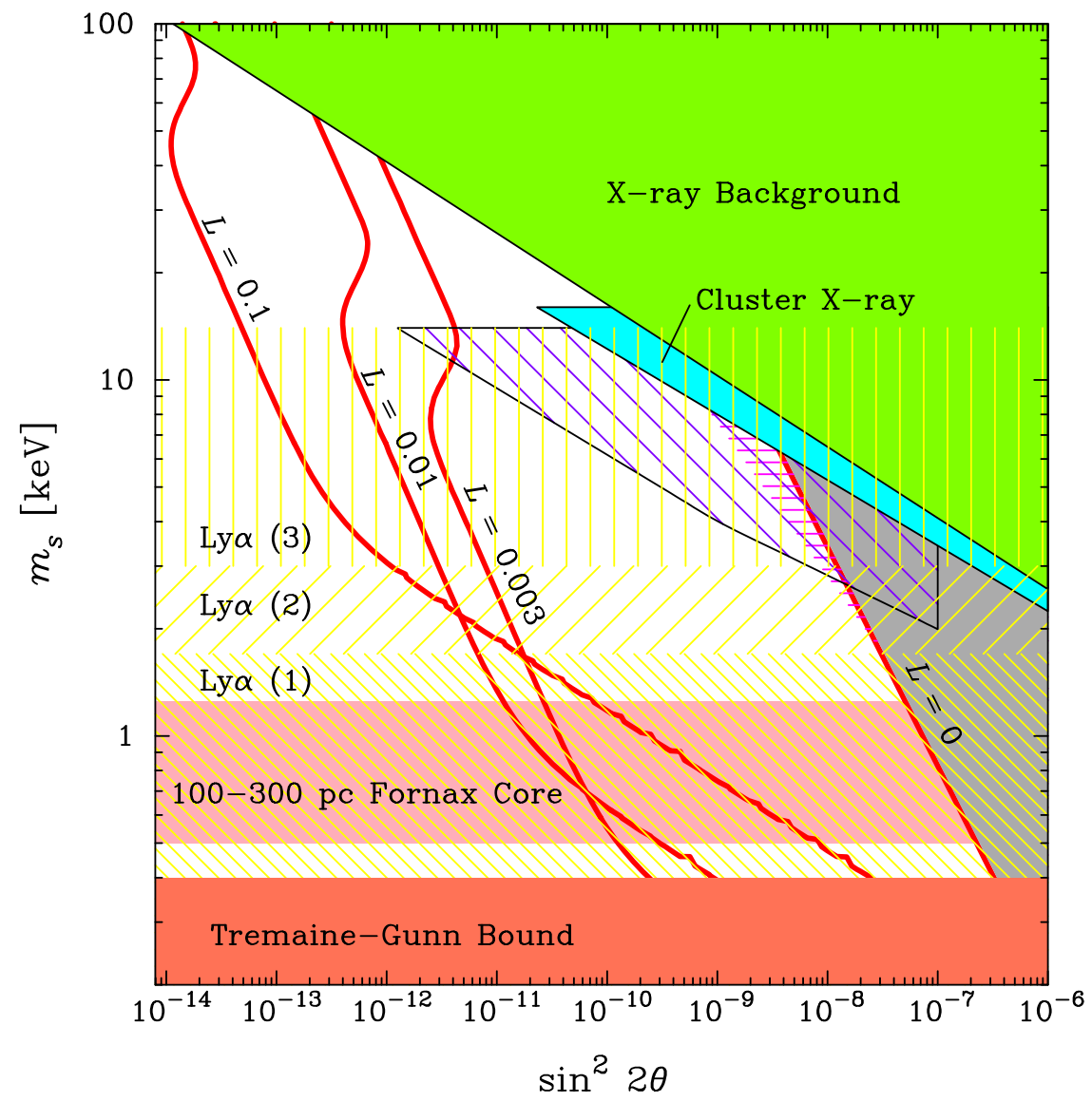

FIG. 20 Regions of the sterile neutrino dark matter $\left(\sin ^{2} 2 \theta, m_{s}\right)$ parameter plane. On the $L=0$ ( $L=0.003,0.01,0.1)$ contours, sterile neutrinos produced by oscillations (resonance production) have relic densities consistent with being all of the dark matter. The grey region to the right of the $L=0$ contour is excluded by overclosure. The regions denoted "X-ray Background" and "Cluster X-ray" are excluded by null searches for X-rays from $\nu_{s} \rightarrow \gamma \nu_{a}$; the diagonal wide-hatched region is the projected reach of future X-ray searches. The "Fornax" and horizontal hatched regions are favored to explain a core in the Fornax dwarf galaxy and pulsar kicks, respectively. The Tremaine-Gunn phase space density bound and a variety of Lyman- $\alpha$ forest constraints on small scale structure place lower bounds on $m_{s}$ as shown. From Ref. (229).

angles and masses, as shown in Fig. 20(230). For further work on the relic density in both the $L=0$ and $L>0$ scenarios, see Ref. (231; 232).

Sterile neutrinos may also be produced at higher temperatures, for example, in the decay of heavy particles. One example follows from introducing a singlet Higgs boson field $S$, which couples to right-handed neutrinos through $-\frac{1}{2} \lambda_{\alpha \beta}^{S} S \bar{\nu}^{\alpha} \nu^{\beta}$. When $S$ gets a vacuum expectation value, this term becomes a Majorana mass term. At temperatures of $T \sim m_{S}$, this term also produces sterile neutrinos through the decays $S \rightarrow \bar{\nu}_{s} \nu_{s}$, and this may be a significant source of colder sterile neutrinos (233; 234; 235). 


\section{B. Indirect Detection}

The dominant decay of sterile neutrinos is through $\nu_{s} \rightarrow \nu_{L} \bar{\nu}_{L} \nu_{L}$. However, sterile neutrinos may also decay through a loop-level process to a photon and an active neutrino with branching ratio $27 \alpha /(8 \pi) \approx 1 / 128$. The radiative decay width is (236)

$$
\Gamma\left(\nu_{s} \rightarrow \gamma \nu_{a}\right)=\frac{9 \alpha}{2048 \pi^{4}} G_{F}^{2} \sin ^{2} 2 \theta m_{s}^{5} \simeq \frac{1}{1.5 \times 10^{32} \mathrm{~s}} \frac{\sin ^{2} 2 \theta}{10^{-10}}\left[\frac{m_{s}}{\mathrm{keV}}\right]^{5} .
$$

For the allowed sterile neutrino parameters, the sterile neutrino's lifetime is much longer than the age of the Universe, as required for it to be dark matter.

At the same time, even if a small fraction of sterile neutrinos decay, this may be observed. Because the radiative decay is two-body, the signal is a mono-energetic flux of X-rays with energy $E_{\gamma} \simeq m_{s} / 2$. Such signals may be seen by the XMM-Newton, Chandra X-ray, and Suzaku observatories. Null results exclude the upper right shaded region of Fig. 20, and these constraints have been updated in later analyses (see, e.g., Ref. (232; 237)). There is also reported evidence of a signal in Chandra data, consistent with $\left(m_{s}, \sin ^{2} 2 \theta\right) \sim(5 \mathrm{keV}, 3 \times$ $10^{-9}$ ), in the heart of the allowed parameter space (238; 239; 240). Future observations from the International X-ray Observatory may extend sensitivities to the entire range of parameters plotted in Fig. $20(237)$.

\section{Astrophysical Signals}

Sterile neutrinos are the classic warm dark matter candidate. Their warmth depends on the production mechanism, however. For the three production mechanisms discussed in Sec. VII.A, the sterile neutrino free-streaming length is roughly

$$
\lambda_{\mathrm{FS}} \approx R \frac{\mathrm{keV}}{m_{s}}
$$

where $R=0.9,0.6$, and $0.2 \mathrm{Mpc}$ for production from oscillations, $L$-enhanced production (depending on $L$ ), and production through Higgs decay, respectively (241). This implies that bounds on small scale structure, for example, through Lyman- $\alpha$ constraints, depend on the production mechanism. For production by oscillations, current bounds require $m_{s} \gtrsim 10 \mathrm{keV}$, effectively excluding this production mechanism as a source for all of dark matter (see Fig. 20. For colder production mechanisms, however, the Lyman- $\alpha$ bounds on $m_{s}$ are weaker, and these mechanisms may viably produce all of the dark matter. We see, though, that for much of sterile neutrino parameter space, power on small scales is reduced, providing an observable difference from vanilla cold dark matter. For further discussion of small scale structure constraints on sterile neutrinos, see Ref. (180; 229; 237; 242).

In addition to its impact on small structure and the X-ray spectrum, sterile neutrinos may have other astrophysical effects, for example, on the velocity distribution of pulsars and on the formation of the first stars. For reviews, see Ref. (241; 243). 


\section{AXIONS}

Axions are motivated by the strong CP problem described in Sec. II.B.4 (244; 245; 246). The axion solution follows from introducing a new pseudoscalar field $a$ with coupling

$$
\mathcal{L}_{a}=-\frac{g_{3}^{2}}{32 \pi^{2}} \frac{a}{f_{a}} \epsilon^{\mu \nu \rho \sigma} G_{\mu \nu}^{\alpha} G_{\rho \sigma}^{\alpha}
$$

where $f_{a}$ is a new mass scale, the axion decay constant. This term makes the coefficient of $\epsilon^{\mu \nu \rho \sigma} G_{\mu \nu}^{\alpha} G_{\rho \sigma}^{\alpha}$ dynamical. The vacuum energy depends on this coefficient, and it relaxes to a minimum where the EDM of the neutron is very small and consistent with current bounds.

As we will see, the allowed parameters for axions imply that they are extremely light and weakly-interacting, providing yet another qualitatively different dark matter candidate well-motivated by particle physics (247, 248; 249). For a general review of axions, see, e.g., Ref. (250).

\section{A. Production Mechanisms}

The axion's mass and interactions are determined by $f_{a}$ up to model-dependent constants that are typically $\mathcal{O}(1)$. The axion's mass is

$$
m_{a}=\frac{\sqrt{m_{u} m_{d}}}{m_{u}+m_{d}} m_{\pi} f_{\pi} \frac{1}{f_{a}} \approx 6 \mu \mathrm{eV}\left(\frac{10^{12} \mathrm{GeV}}{f_{a}}\right),
$$

where $m_{u} \simeq 4 \mathrm{MeV}, m_{d} \simeq 8 \mathrm{MeV}$, and $m_{\pi} \simeq 135 \mathrm{MeV}$ are the up quark, down quark, and pion masses, and $f_{\pi} \simeq 93 \mathrm{MeV}$ is the pion decay constant.

Axions interact with gluons, through the term of Eq. (47), and also with fermions. At loop-level, they also interact with photons through the coupling

$$
\mathcal{L}_{a \gamma \gamma}=-g_{\gamma} \frac{\alpha}{\pi} \frac{a}{f_{a}} \vec{E} \cdot \vec{B} \equiv-g_{a \gamma \gamma} a \vec{E} \cdot \vec{B}
$$

where $\alpha$ is the fine-structure constant and $g_{\gamma}$ is a model-dependent parameter. For two well-known possibilities, the KSVZ (251; 252) and DFSZ (253; 254) axions, $g_{\gamma}$ is -0.97 and 0.36 , respectively.

The axion's mass is bounded by several independent constraints. The coupling of Eq. (49) implies that axions decay with lifetime

$$
\tau(a \rightarrow \gamma \gamma)=\frac{64 \pi}{g_{a \gamma \gamma}^{2} m_{a}^{3}} \simeq \frac{8.8 \times 10^{23} \mathrm{~s}}{g_{\gamma}^{2}}\left(\frac{\mathrm{eV}}{m_{a}}\right)^{5}
$$

For axions to live longer than the age of the Universe, $m_{a} \lesssim 20 \mathrm{eV}$. Other astrophysical bounds are even more stringent. In particular, axions may be produced in astrophysical bodies and then escape, leading to a new source of energy loss. Constraints from the longevity of red giants and the observed length of the neutrino pulse from Supernova 1987a, along with other astrophysical constraints, require $f_{a} \gtrsim 10^{9} \mathrm{GeV}$, implying $m_{a} \lesssim 10 \mathrm{meV}$ (255).

There are several possible production mechanisms for axion dark matter. A priori the most straightforward is thermal production, as in the case of light gravitinos and sterile neutrinos. Unfortunately, axions produced thermally would have a relic density of $\Omega_{a}^{\text {th }} \sim$ 
$0.22\left(m_{a} / 80 \mathrm{eV}\right)$ and be hot dark matter. In addition, Eq. (50) implies that axions with mass $\sim 80 \mathrm{eV}$ have lifetimes shorter than the age of the Universe, and so this mechanism cannot produce axions that are the bulk of dark matter.

There are, however, several non-thermal production mechanisms linked to the rich cosmological history of the axion field. As the Universe cools to a temperature $T \sim f_{a}$, the axion field takes values that vary from place to place. This is known as the Peccei-Quinn (PQ) phase transition. Although the value of the axion field now is fixed to minimize the vacuum energy and solve the strong $\mathrm{CP}$ problem, at temperatures $T \gtrsim \mathrm{GeV}$, other effects dominate the vacuum energy, and all values of the axion field are equally possible.

If inflation occurs after the PQ phase transition, then our observable Universe lies in a patch with a single value of the axion field. At $T \sim \mathrm{GeV}$, the axion field then relaxes to its minimum. This "vacuum realignment" generates a relic density (256)

$$
\Omega_{a} \simeq 0.4 \theta_{i}^{2}\left(\frac{f_{a}}{10^{12} \mathrm{GeV}}\right)^{1.18},
$$

where $\theta_{i}$ is the initial vacuum misalignment angle, assuming the relic density is not diluted by late entropy production. The requirement $\Omega_{a} \leq \Omega_{\mathrm{DM}}$ implies $f_{a} \lesssim 10^{12} \mathrm{GeV} \theta_{i}^{-2}$.

On the other hand, inflation may occur before the PQ phase transition. This has two effects. Our observable Universe then consists of a mixture of many patches with different $\theta_{i}$, and the relic density from vacuum re-alignment is that given in Eq. (51), but with an effective $\theta_{i} \sim \mathcal{O}(1)$. In addition, since many different patches are observable, the boundaries between patches, topological defects such as domain walls and axionic strings generated during the PQ phase transition, may have observable effects. Production from domain wall decay is expected to be sub-dominant (257) to vacuum realignment, but the relic density of axions radiated by axionic strings may be of the same order or even an order of magnitude larger (258; 259; 260).

To summarize, if inflation occurs after the PQ transition, the allowed window of axion parameter space is roughly

$$
\begin{aligned}
10^{12} \mathrm{GeV} \theta_{i}^{-2} & \gtrsim f_{a} \\
6 \mu \mathrm{eV} \theta_{i}^{2} & \lesssim m_{a} \lesssim 6 \mathrm{meV},
\end{aligned}
$$

where $\theta_{i}$ is an arbitrary constant less than 1 . If inflation occurs before, then Eq. (52) applies with $\theta_{i} \sim 1$, and axion string production may imply a slightly stronger upper bound on $f_{a}$.

The lower bound on $m_{a}$ arises from requiring that axions don't overclose the Universe. When this bound is saturated, axions may be all of the dark matter, and so this is the preferred target region for dark matter searches. In contrast to WIMPs and superWIMPs, axions do not naturally have the correct relic density: there is a range of possible $m_{a}$, and there is no reason a priori to be in the allowed window or near it's lower boundary.

Note, however, that, if inflation occurred after the PQ transition, the lower bound on $m_{a}$ depends sensitively on $\theta_{i}$. In this case, if $\theta_{i} \ll 1$, axions may be all of the dark matter even for smaller $m_{a}$ and larger $f_{a}$. This latter possibility has some theoretical attractions, as it implies $f_{a}$ near $m_{\mathrm{GUT}} \simeq 10^{16} \mathrm{GeV}$ may be allowed and provides an avenue for anthropic selection effects to favor axion densities near the observed value (261; 262). As we will see, however, if axions are even lighter and more weakly-coupled axions than naively expected, they will be beyond detection for the foreseeable future. 


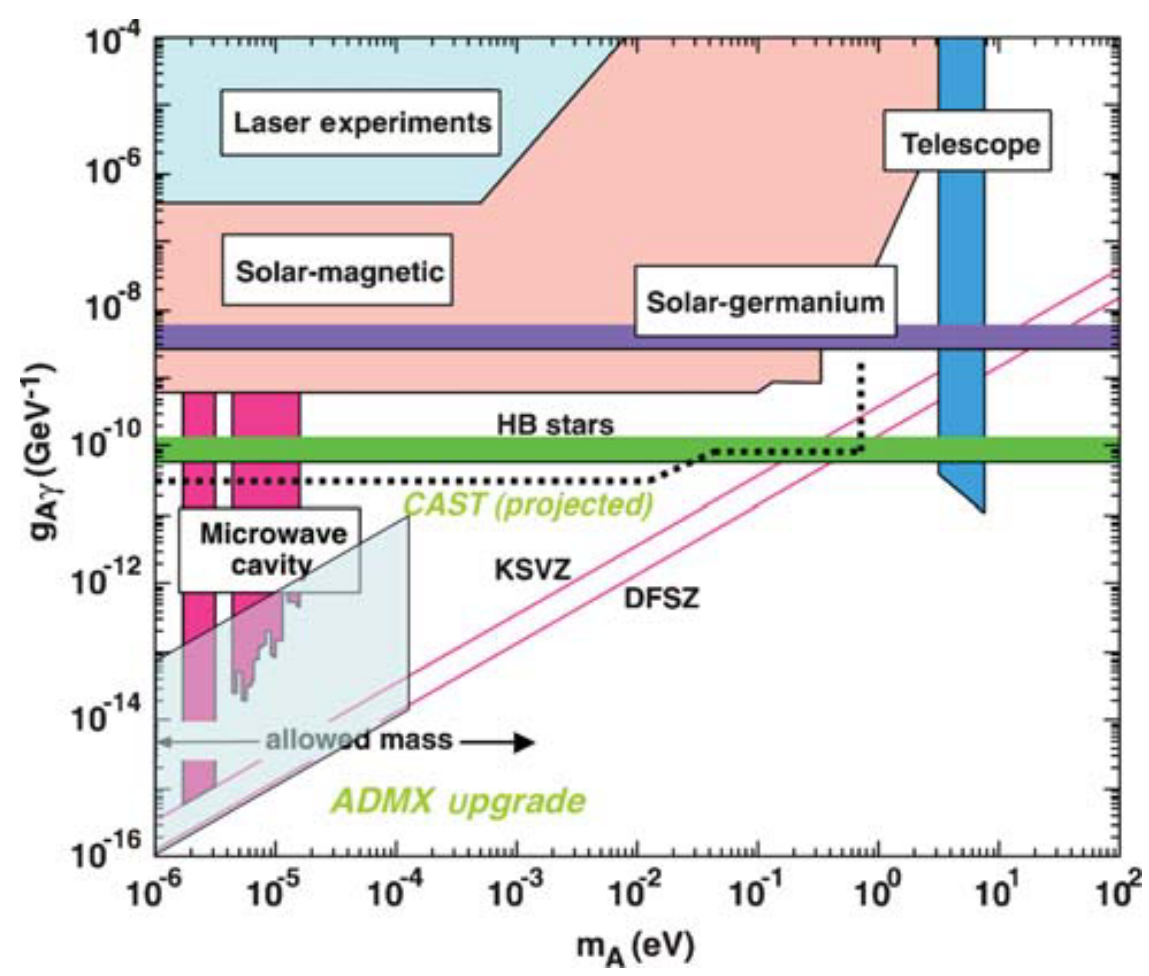

FIG. 21 Current constraints (shaded) and theoretical predictions for KSVZ and DFSZ axions in the $\left(m_{a}, g_{A \gamma}=g_{a \gamma \gamma}\right)$ plane. "Allowed mass" denotes roughly the window of axion masses allowed by Eq. (52); see text for details. From Ref. (250).

\section{B. Direct Detection}

Axions may be detected directly by looking for their scattering with SM particles in the laboratory. Current and projected limits from direct detection axion experiments are shown in Fig. 21, along with the theoretical predictions for KSVZ and DFSZ axions.

For cosmological axions, given all of the caveats discussed above, the favored region of axion parameter space, in which axions may be all of the dark matter, may be taken to be $1 \mu \mathrm{eV} \lesssim m_{a} \lesssim 100 \mu \mathrm{eV}$. In this region, the leading experimental results are from the Axion Dark Matter Experiment (ADMX). ADMX searches for cosmological axions by looking for the resonantly-enhanced conversion of dark matter axions to photons through scattering off a background magnetic field, the Primakoff process $a \gamma^{*} \rightarrow \gamma(263)$. This is a scanning experiment - one must run at a given frequency to be sensitive to axions of a given mass. Once the desired sensitivity in coupling $g_{a \gamma \gamma}$ has been reached, one then moves to another frequency. Theoretical expectations therefore play a role in setting the search strategy. To date, ADMX has probed down to the level of KSVZ predictions for axion masses of a few $\mu \mathrm{eV}$. In the coming decade, ADMX will continue running, along with another experiment, New CARRACK, also based on the Primakoff process. ADMX expects to extend its sensitivity to DFSZ predictions for $\mu \mathrm{eV}<m_{a}<100 \mu \mathrm{eV}$.

In addition to searches for cosmological axions, there are also searches for axions produced in the core of the Sun. These and other experiments are reviewed in Ref. (250). 


\section{CONCLUSIONS}

Current observations support a remarkably simple model of the Universe consisting of baryons, dark matter, and dark energy, supplemented by initial conditions determined by an early epoch of inflation. If scientific progress is characterized by periods of confusion, which are resolved by neat and tidy models, which are then launched back into confusion by further data, the current era is most definitely of the neat and tidy sort.

Dark matter may be the area that launches us back into confusion with further data. The microscopic properties of dark matter are as much of a mystery now as they were in the 1930's. In the next few years, however, searches for dark matter through a variety of means discussed here will discover or exclude many of the most promising candidates.

At its core, the dark matter problem is highly interdisciplinary. Rather than attempt a summary of this review, we close with some optimistic, but plausible, scenarios for the future in which experiments from both particle physics and astrophysics are required to identify dark matter. Consider the following examples:

- Scenario 1: Direct detection experiments see a dark matter signal in spin-independent scattering. This result is confirmed by the LHC, which sees a missing energy signal that is followed up by precision measurements pointing to a $800 \mathrm{GeV}$ Kaluza-Klein gauge boson. Further LHC studies constrain the Kaluza-Klein particle's predicted thermal relic density to be identical at the percent level with $\Omega_{\mathrm{DM}}$, establishing a new standard cosmology in which the dark matter is composed entirely of Kaluza-Klein dark matter, cosmology is standard back to 1 ns after the Big Bang, and the Universe has extra dimensions. Direct and indirect detection rates are then used to constrain halo profiles and substructure, ushering in a new era of dark matter astronomy.

- Scenario 2: The LHC discovers heavy, charged particles that are apparently stable. Together the LHC and International Linear Collider determine that the new particles are staus, predicted by supersymmetry. Detailed follow-up studies show that, if these staus are absolutely stable, their thermal relic density is larger than the total mass of the Universe! This paradox is resolved by further studies that show that staus decay on time scales of a month to gravitinos. Careful studies of the decays determine that the amount of gravitinos in the Universe is exactly that required to be dark matter, providing strong quantitative evidence that dark matter is entirely in the form of gravitinos, and providing empirical support for supergravity and string theory.

- Scenario 3: An X-ray experiment discovers a line signal. Assuming this results from decaying sterile neutrinos, the photon energy determines the neutrino's mass $m=2 E_{\gamma}$, the intensity determines the neutrino mixing angle $\left(I \propto \sin ^{2} \theta\right)$, and the image morphology determines the dark matter's spatial distribution. From the mass and radial distribution, theorists determine the free-streaming length. This favors production from Higgs decays over production by oscillations, leading to predictions of nonstandard Higgs phenomenology, which are then confirmed at the LHC. Additional information on neutrino parameters from the LHC strengthens the hypothesis of sterile neutrino dark matter, and the energy distribution of the narrow spectral line is then used to study the expansion history of the Universe and dark energy.

These scenarios are, of course, highly speculative and idealized, but they illustrate that, even in ideal scenarios that we have studied and understand, close interactions between many subfields will be required. At the same time, if any of the ideas discussed here is 
correct, there are promising prospects for the combination of detection methods in particle physics and astrophysics to identify dark matter in the not-so-distant future.

\section{ACKNOWLEDGMENTS}

I am grateful to my colleagues and collaborators at UC Irvine and elsewhere for their many valuable insights. I also thank the participants of the Keck Institute for Space Studies program "Shedding Light on the Nature of Dark Matter" for stimulating interactions, and Alex Kusenko, David Sanford, and Hai-Bo Yu for comments on this manuscript. This work was supported in part by NSF Grant No. PHY-0653656.

\section{References}

[1] F. Zwicky, "Spectral displacement of extra galactic nebulae," Helv. Phys. Acta 6 (1933) 110-127.

[2] V. C. Rubin and W. K. Ford, Jr., "Rotation of the Andromeda Nebula from a Spectroscopic Survey of Emission Regions," Astrophys. J. 159 (1970) 379-403.

[3] V. C. Rubin, N. Thonnard, and W. K. Ford, Jr., "Rotational properties of 21 SC galaxies with a large range of luminosities and radii, from NGC $4605(\mathrm{R}=4 \mathrm{kpc})$ to UGC 2885 (R $=122 \mathrm{kpc}), "$ Astrophys. J. 238 (1980) 471.

[4] A. Bosma, The distribution and kinematics of neutral hydrogen in spiral galaxies of various morphological types, PhD Thesis, Groningen Univ. (1978).

[5] V. Trimble, "Existence and Nature of Dark Matter in the Universe," Ann. Rev. Astron. Astrophys. 25 (1987) 425-472.

[6] A. Refregier, "Weak Gravitational Lensing by Large-Scale Structure," Ann. Rev. Astron. Astrophys. 41 (2003) 645-668, arXiv:astro-ph/0307212

[7] J. A. Tyson, G. P. Kochanski, and I. P. Dell'Antonio, "Detailed Mass Map of CL0024+1654 from Strong Lensing," Astrophys. J. 498 (1998) L107, arXiv:astro-ph/9801193.

[8] A. D. Lewis, D. A. Buote, and J. T. Stocke, "Chandra Observations of Abell 2029: The Dark Matter Profile at $<0.01 R_{\text {vir }}$ in an Unusually Relaxed Cluster," Astrophys. J. 586 (2003) 135-142, arXiv:astro-ph/0209205.

[9] D. Clowe et al., "A direct empirical proof of the existence of dark matter," Astrophys. J. 648 (2006) L109-L113, arXiv:astro-ph/0608407.

[10] Particle Data Group Collaboration, B. D. Fields and S. Sarkar, "Big Bang nucleosynthesis," Phys. Lett. B667 (2008) 228-231.

[11] S. W. Allen, A. C. Fabian, R. W. Schmidt, and H. Ebeling, "Cosmological constraints from the local X-ray luminosity function of the most X-ray luminous galaxy clusters," Mon. Not. Roy. Astron. Soc. 342 (2003) 287, arXiv:astro-ph/0208394.

[12] Supernova Search Team Collaboration, A. G. Riess et al., "Observational Evidence from Supernovae for an Accelerating Universe and a Cosmological Constant," Astron. J. 116 (1998) 1009-1038, arXiv:astro-ph/9805201.

[13] Supernova Cosmology Project Collaboration, S. Perlmutter et al., "Measurements of Omega and Lambda from 42 High-Redshift Supernovae," Astrophys. J. 517 (1999) 565-586, arXiv:astro-ph/9812133. 
[14] E. Komatsu et al., "Seven-Year Wilkinson Microwave Anisotropy Probe (WMAP) Observations: Cosmological Interpretation," arXiv:1001.4538 [astro-ph.CO].

[15] M. Herrero, "The Standard model," arXiv:hep-ph/9812242.

[16] ALEPH, DELPHI, L3, and OPAL Collaborations, LEP Higgs Boson Working Group, "Search for the standard model Higgs boson at LEP," Phys. Lett. B565 (2003) 61-75, arXiv:hep-ex/0306033.

[17] ALEPH, DELPHI, L3, and OPAL Collaborations, LEP Electroweak Working Group, "Indirect Constraints on the Mass of the SM Higgs Boson," http://lepewwg.web.cern.ch/LEPEWWG.

[18] CDF and D0 Collaborations, T. Aaltonen et al., "Combination of Tevatron searches for the standard model Higgs boson in the W+W- decay mode," Phys. Rev. Lett. 104 (2010) 061802, arXiv:1001.4162 [hep-ex].

[19] L. Reina, "TASI 2004 lecture notes on Higgs boson physics," arXiv:hep-ph/0512377.

[20] C. T. Hill and E. H. Simmons, "Strong dynamics and electroweak symmetry breaking," Phys. Rept. 381 (2003) 235-402, arXiv: hep-ph/0203079.

[21] Super-Kamiokande Collaboration, Y. Fukuda et al., "Evidence for oscillation of atmospheric neutrinos," Phys. Rev. Lett. 81 (1998) 1562-1567, arXiv:hep-ex/9807003

[22] SNO Collaboration, Q. R. Ahmad et al., "Direct evidence for neutrino flavor transformation from neutral-current interactions in the Sudbury Neutrino Observatory," Phys. Rev. Lett. 89 (2002) 011301, arXiv:nucl-ex/0204008.

[23] C. A. Baker et al., "An improved experimental limit on the electric dipole moment of the neutron," Phys. Rev. Lett. 97 (2006) 131801, arXiv:hep-ex/0602020.

[24] K. Hagiwara, A. D. Martin, D. Nomura, and T. Teubner, "Improved predictions for $g-2$ of the muon and $\alpha_{\mathrm{QED}}\left(M_{Z}^{2}\right)$," Phys. Lett. B649 (2007) 173-179, arXiv:hep-ph/0611102.

[25] Y. B. Zeldovich Adv. Astron. Astrophys. 3 (1965) 241.

[26] H.-Y. Chiu, "Symmetry between particle and anti-particle populations in the universe," Phys. Rev. Lett. 17 (1966) 712.

[27] G. Steigman, "Cosmology Confronts Particle Physics," Ann. Rev. Nucl. Part. Sci. 29 (1979) 313-338.

[28] R. J. Scherrer and M. S. Turner, "On the Relic, Cosmic Abundance of Stable Weakly Interacting Massive Particles," Phys. Rev. D33 (1986) 1585.

[29] HEPAP LHC/ILC Subpanel, "Discovering the Quantum Universe," http://www.science.doe.gov/hep/panels/reports/hepap_reports.shtml.

[30] R. Barbieri and A. Strumia, "What is the limit on the Higgs mass?," Phys. Lett. B462 (1999) 144-149, arXiv: hep-ph/9905281.

[31] J. Wudka, "Natural and model-independent conditions for evading the limits on the scale of new physics," arXiv:hep-ph/0307339.

[32] H.-C. Cheng and I. Low, "TeV symmetry and the little hierarchy problem," JHEP 09 (2003) 051, arXiv:hep-ph/0308199.

[33] G. R. Farrar and P. Fayet, "Phenomenology of the Production, Decay, and Detection of New Hadronic States Associated with Supersymmetry," Phys. Lett. B76 (1978) 575-579.

[34] Y. Shadmi and Y. Shirman, "Dynamical supersymmetry breaking," Rev. Mod. Phys. 72 (2000) 25-64, arXiv:hep-th/9907225.

[35] H. Goldberg, "Constraint on the photino mass from cosmology," Phys. Rev. Lett. 50 (1983) 1419 .

[36] J. R. Ellis, J. S. Hagelin, D. V. Nanopoulos, K. A. Olive, and M. Srednicki, 
"Supersymmetric relics from the big bang," Nucl. Phys. B238 (1984) 453-476.

[37] T. Falk, K. A. Olive, and M. Srednicki, "Heavy Sneutrinos as Dark Matter," Phys. Lett. B339 (1994) 248-251, arXiv: hep-ph/9409270.

[38] C. Arina and N. Fornengo, "Sneutrino cold dark matter, a new analysis: Relic abundance and detection rates," JHEP 11 (2007) 029, arXiv:0709.4477 [hep-ph].

[39] S. Dimopoulos, S. Raby, and F. Wilczek, "Supersymmetry and the Scale of Unification," Phys. Rev. D24 (1981) 1681-1683.

[40] J. L. Feng, K. T. Matchev, and F. Wilczek, "Prospects for indirect detection of neutralino dark matter," Phys. Rev. D63 (2001) 045024, arXiv:astro-ph/0008115

[41] R. Trotta, F. Feroz, M. P. Hobson, L. Roszkowski, and R. Ruiz de Austri, "The Impact of priors and observables on parameter inferences in the Constrained MSSM," JHEP 12 (2008) 024, arXiv:0809.3792 [hep-ph].

[42] O. Klein, "Quantum theory and five-dimensional theory of relativity," Z. Phys. 37 (1926) 895-906.

[43] T. Appelquist, H.-C. Cheng, and B. A. Dobrescu, "Bounds on universal extra dimensions," Phys. Rev. D64 (2001) 035002, arXiv:hep-ph/0012100.

[44] T. Appelquist and H.-U. Yee, "Universal extra dimensions and the Higgs boson mass," Phys. Rev. D67 (2003) 055002, arXiv:hep-ph/0211023

[45] G. Servant and T. M. P. Tait, "Is the lightest Kaluza-Klein particle a viable dark matter candidate?," Nucl. Phys. B650 (2003) 391-419, arXiv: hep-ph/0206071.

[46] H.-C. Cheng, J. L. Feng, and K. T. Matchev, "Kaluza-Klein dark matter," Phys. Rev. Lett. 89 (2002) 211301, arXiv:hep-ph/0207125.

[47] F. Burnell and G. D. Kribs, "The abundance of Kaluza-Klein dark matter with coannihilation," Phys. Rev. D73 (2006) 015001, arXiv: hep-ph/0509118.

[48] K. Kong and K. T. Matchev, "Precise calculation of the relic density of Kaluza-Klein dark matter in universal extra dimensions," JHEP 01 (2006) 038, arXiv:hep-ph/0509119.

[49] M. Kakizaki, S. Matsumoto, and M. Senami, "Relic abundance of dark matter in the minimal universal extra dimension model," Phys. Rev. D74 (2006) 023504, arXiv:hep-ph/0605280.

[50] J. A. R. Cembranos, A. Dobado, and A. L. Maroto, "Brane-world dark matter," Phys. Rev. Lett. 90 (2003) 241301, arXiv:hep-ph/0302041.

[51] J. A. R. Cembranos, A. Dobado, and A. L. Maroto, "Cosmological and astrophysical limits on brane fluctuations," Phys. Rev. D68 (2003) 103505, arXiv:hep-ph/0307062.

[52] A. Birkedal, A. Noble, M. Perelstein, and A. Spray, "Little Higgs dark matter," Phys. Rev. D74 (2006) 035002, arXiv:hep-ph/0603077.

[53] K. Agashe and G. Servant, "Warped unification, proton stability and dark matter," Phys. Rev. Lett. 93 (2004) 231805, arXiv:hep-ph/0403143.

[54] G. D. Mack, J. F. Beacom, and G. Bertone, "Towards Closing the Window on Strongly Interacting Dark Matter: Far-Reaching Constraints from Earth's Heat Flow," Phys. Rev. D76 (2007) 043523, arXiv:0705.4298 [astro-ph].

[55] I. F. M. Albuquerque and C. P. d. l. Heros, "Closing the Window on Strongly Interacting Dark Matter with IceCube," arXiv:1001.1381 [astro-ph.HE].

[56] HEPAP/AAAC DMSAG Subpanel, "Final Report of the Dark Matter Scientific Assessment Group," http://www.science.doe.gov/hep/panels/reports/hepap_reports.shtml.

[57] R. J. Gaitskell, "Direct detection of dark matter," Ann. Rev. Nucl. Part. Sci. 54 (2004) 315-359. 
[58] M. W. Goodman and E. Witten, "Detectability of certain dark-matter candidates," Phys. Rev. D31 (1985) 3059.

[59] A. M. Green, "Effect of halo modelling on WIMP exclusion limits," Phys. Rev. D66 (2002) 083003, arXiv:astro-ph/0207366.

[60] M. Kamionkowski and S. M. Koushiappas, "Galactic Substructure and Direct Detection of Dark Matter," Phys. Rev. D77 (2008) 103509, arXiv:0801.3269 [astro-ph].

[61] XENON Collaboration, J. Angle et al., "First Results from the XENON10 Dark Matter Experiment at the Gran Sasso National Laboratory," Phys. Rev. Lett. 100 (2008) 021303, arXiv:0706.0039 [astro-ph].

[62] E. Aprile et al., "New Measurement of the Relative Scintillation Efficiency of Xenon Nuclear Recoils Below $10 \mathrm{keV,"} \mathrm{Phys.} \mathrm{Rev.} \mathrm{C79} \mathrm{(2009)} \mathrm{045807,} \mathrm{arXiv:0810.0274} \mathrm{[astro-ph].}$

[63] V. N. Lebedenko et al., "Result from the First Science Run of the ZEPLIN-III Dark Matter Search Experiment," Phys. Rev. D80 (2009) 052010, arXiv:0812.1150 [astro-ph].

[64] E. Armengaud et al., "First results of the EDELWEISS-II WIMP search using Ge cryogenic detectors with interleaved electrodes," arXiv:0912.0805 [astro-ph.C0].

[65] CDMS-II Collaboration, Z. Ahmed et al., "Results from the Final Exposure of the CDMS II Experiment," arXiv:0912.3592 [astro-ph.CO].

[66] DAMA Collaboration, R. Bernabei et al., "First results from DAMA/LIBRA and the combined results with DAMA/NaI," Eur. Phys. J. C56 (2008) 333-355, arXiv:0804. 2741 [astro-ph].

[67] C. Savage, G. Gelmini, P. Gondolo, and K. Freese, "Compatibility of DAMA/LIBRA dark matter detection with other searches," JCAP 0904 (2009) 010, arXiv:0808.3607 [astro-ph].

[68] Y. G. Kim, T. Nihei, L. Roszkowski, and R. Ruiz de Austri, "Upper and lower limits on neutralino WIMP mass and spin- independent scattering cross section, and impact of new (g- 2)(mu) measurement," JHEP 12 (2002) 034, arXiv:hep-ph/0208069.

[69] R. J. Gaitskell, V. Mandic, and J. Filippini, "Dark Matter Tools," http://dmtools.brown.edu.

[70] CDMS Collaboration, Z. Ahmed et al., "Search for Weakly Interacting Massive Particles with the First Five-Tower Data from the Cryogenic Dark Matter Search at the Soudan Underground Laboratory," Phys. Rev. Lett. 102 (2009) 011301, arXiv:0802.3530 [astro-ph].

[71] COUPP Collaboration, E. Behnke et al., "Improved Spin-Dependent WIMP Limits from a Bubble Chamber," Science 319 (2008) 933-936, arXiv:0804.2886 [astro-ph].

[72] KIMS Collaboration, H. S. Lee. et al., "Limits on WIMP-nucleon cross section with CsI(Tl) crystal detectors," Phys. Rev. Lett. 99 (2007) 091301, arXiv:0704.0423 [astro-ph].

[73] Super-Kamiokande Collaboration, S. Desai et al., "Search for dark matter WIMPs using upward through-going muons in Super-Kamiokande," Phys. Rev. D70 (2004) 083523, arXiv:hep-ex/0404025.

[74] IceCube Collaboration, R. Abbasi et al., "Limits on a muon flux from neutralino annihilations in the Sun with the IceCube 22-string detector," Phys. Rev. Lett. 102 (2009) 201302, arXiv:0902.2460 [astro-ph.CO].

[75] IceCube Collaboration, J. Braun and D. Hubert, "Searches for WIMP Dark Matter from the Sun with AMANDA," arXiv:0906.1615 [astro-ph.HE].

[76] J. L. Feng, K. T. Matchev, and T. Moroi, "Multi-TeV scalars are natural in minimal supergravity," Phys. Rev. Lett. 84 (2000) 2322-2325, arXiv:hep-ph/9908309. 
[77] J. L. Feng, K. T. Matchev, and T. Moroi, "Focus points and naturalness in supersymmetry," Phys. Rev. D61 (2000) 075005, arXiv:hep-ph/9909334.

[78] J. L. Feng, K. T. Matchev, and F. Wilczek, "Neutralino dark matter in focus point supersymmetry," Phys. Lett. B482 (2000) 388-399, arXiv: hep-ph/0004043.

[79] A. K. Drukier, K. Freese, and D. N. Spergel, "Detecting Cold Dark Matter Candidates," Phys. Rev. D33 (1986) 3495-3508.

[80] D. Tucker-Smith and N. Weiner, "Inelastic dark matter," Phys. Rev. D64 (2001) 043502, arXiv:hep-ph/0101138.

[81] P. Gondolo and G. Gelmini, "Compatibility of DAMA dark matter detection with other searches," Phys. Rev. D71 (2005) 123520, arXiv: hep-ph/0504010.

[82] E. M. Drobyshevski, "Channeling Effect and Improvement of the Efficiency of Charged Particle Registration with Crystal Scintillators," Mod. Phys. Lett. A23 (2008) 3077-3085, arXiv:0706.3095 [physics.ins-det].

[83] R. Bernabei et al., "Possible implications of the channeling effect in NaI(Tl) crystals," Eur. Phys. J. C53 (2008) 205-213, arXiv:0710.0288 [astro-ph].

[84] CoGeNT Collaboration, C. E. Aalseth et al., "Results from a Search for Light-Mass Dark Matter with a P- type Point Contact Germanium Detector," arXiv:1002.4703 [astro-ph.CO].

[85] H. K. Dreiner et al., "Mass Bounds on a Very Light Neutralino," Eur. Phys. J. C62 (2009) 547-572, arXiv:0901.3485 [hep-ph].

[86] A. Bottino, F. Donato, N. Fornengo, and S. Scopel, "Zooming in on light relic neutralinos by direct detection and measurements of galactic antimatter," Phys. Rev. D77 (2008) 015002, arXiv:0710.0553 [hep-ph].

[87] D. Hooper, F. Petriello, K. M. Zurek, and M. Kamionkowski, "The New DAMA Dark-Matter Window and Energetic-Neutrino Searches," Phys. Rev. D79 (2009) 015010 arXiv:0808.2464 [hep-ph].

[88] J. L. Feng, J. Kumar, J. Learned, and L. E. Strigari, "Testing the Dark Matter Interpretation of the DAMA/LIBRA Result with Super-Kamiokande," JCAP 0901 (2009) 032 , arXiv:0808.4151 [hep-ph].

[89] J. Kumar, J. G. Learned, and S. Smith, "Light Dark Matter Detection Prospects at Neutrino Experiments," Phys. Rev. D80 (2009) 113002, arXiv:0908.1768 [hep-ph].

[90] PAMELA Collaboration, O. Adriani et al., "An anomalous positron abundance in cosmic rays with energies 1.5.100 GeV," Nature 458 (2009) 607-609, arXiv:0810.4995 [astro-ph].

[91] J. Chang et al., "An excess of cosmic ray electrons at energies of $300.800 \mathrm{GeV}$," Nature 456 (2008) 362-365.

[92] Fermi LAT Collaboration, A. A. Abdo et al., "Measurement of the Cosmic Ray e+ plus espectrum from $20 \mathrm{GeV}$ to $1 \mathrm{TeV}$ with the Fermi Large Area Telescope," Phys. Rev. Lett. 102 (2009) 181101, arXiv:0905.0025 [astro-ph.HE]

[93] A. W. Strong et al., "The GALPROP Cosmic-Ray Propagation Code," arXiv:0907.0559 [astro-ph.HE].

[94] FERMI-LAT Collaboration, D. Grasso et al., "On possible interpretations of the high energy electron- positron spectrum measured by the Fermi Large Area Telescope," Astropart. Phys. 32 (2009) 140-151, arXiv:0905.0636 [astro-ph.HE]

[95] A. Boulares, "The nature of the cosmic-ray electron spectrum, and supernova remnant contributions," Astrophys. J. 342 (July, 1989) 807-813. 
[96] L. Zhang and K. S. Cheng, "Cosmic-ray positrons from mature gamma-ray pulsars," Astron. Astrophys. 368 (Mar., 2001) 1063-1070.

[97] D. Hooper, P. Blasi, and P. D. Serpico, "Pulsars as the Sources of High Energy Cosmic Ray Positrons," JCAP 0901 (2009) 025, arXiv:0810.1527 [astro-ph].

[98] H. Yuksel, M. D. Kistler, and T. Stanev, "TeV Gamma Rays from Geminga and the Origin of the GeV Positron Excess," Phys. Rev. Lett. 103 (2009) 051101, arXiv:0810.2784 [astro-ph].

[99] S. Profumo, "Dissecting Pamela (and ATIC) with Occam's Razor: existing, well-known Pulsars naturally account for the 'anomalous' Cosmic-Ray Electron and Positron Data," arXiv:0812.4457 [astro-ph].

[100] S. Dado and A. Dar, "Dark Matter Signals In Cosmic Rays?," arXiv:0903.0165 [astro-ph.HE].

[101] P. L. Biermann et al., "Cosmic Ray Electrons and Positrons from Supernova Explosions of Massive Stars," Phys. Rev. Lett. 103 (2009) 061101, arXiv:0903.4048 [astro-ph.HE].

[102] B. Katz, K. Blum, and E. Waxman, "What can we really learn from positron flux 'anomalies'?," arXiv:0907.1686 [astro-ph.HE].

[103] L. Bergstrom, J. Edsjo, and G. Zaharijas, "Dark matter interpretation of recent electron and positron data," Phys. Rev. Lett. 103 (2009) 031103, arXiv:0905.0333 [astro-ph.HE].

[104] J. L. Feng, M. Kaplinghat, and H.-B. Yu, "Halo Shape and Relic Density Exclusions of Sommerfeld- Enhanced Dark Matter Explanations of Cosmic Ray Excesses," arXiv:0911.0422 [hep-ph].

[105] A. Sommerfeld Annalen der Physik 403 (1931) 207.

[106] J. Hisano, S. Matsumoto, and M. M. Nojiri, "Unitarity and higher-order corrections in neutralino dark matter annihilation into two photons," Phys. Rev. D67 (2003) 075014, arXiv:hep-ph/0212022.

[107] M. Cirelli, A. Strumia, and M. Tamburini, "Cosmology and Astrophysics of Minimal Dark Matter," Nucl. Phys. B787 (2007) 152-175, arXiv:0706.4071 [hep-ph].

[108] M. Cirelli, M. Kadastik, M. Raidal, and A. Strumia, "Model-independent implications of the e+, e-, anti-proton cosmic ray spectra on properties of Dark Matter," Nucl. Phys. B813 (2009) 1-21, arXiv:0809.2409 [hep-ph].

[109] N. Arkani-Hamed, D. P. Finkbeiner, T. R. Slatyer, and N. Weiner, "A Theory of Dark Matter," Phys. Rev. D79 (2009) 015014, arXiv:0810.0713 [hep-ph].

[110] M. Kamionkowski and S. Profumo, "Early Annihilation and Diffuse Backgrounds in Models of Weakly Interacting Massive Particles in Which the Cross Section for Pair Annihilation Is Enhanced by 1/v," Phys. Rev. Lett. 101 (2008) 261301, arXiv:0810.3233 [astro-ph].

[111] J. B. Dent, S. Dutta, and R. J. Scherrer, "Thermal Relic Abundances of Particles with Velocity- Dependent Interactions," arXiv:0909.4128 [astro-ph.C0].

[112] J. Zavala, M. Vogelsberger, and S. D. M. White, "Relic density and CMB constraints on dark matter annihilation with Sommerfeld enhancement," arXiv:0910.5221 [astro-ph.CO].

[113] D. Feldman, Z. Liu, and P. Nath, "PAMELA Positron Excess as a Signal from the Hidden Sector," Phys. Rev. D79 (2009) 063509, arXiv:0810.5762 [hep-ph].

[114] M. Ibe, H. Murayama, and T. T. Yanagida, "Breit-Wigner Enhancement of Dark Matter Annihilation," Phys. Rev. D79 (2009) 095009, arXiv:0812.0072 [hep-ph].

[115] W.-L. Guo and Y.-L. Wu, "Enhancement of Dark Matter Annihilation via Breit-Wigner 
Resonance," Phys. Rev. D79 (2009) 055012, arXiv:0901.1450 [hep-ph].

[116] M. Ibe and H.-B. Yu, "Distinguishing Dark Matter Annihilation Enhancement Scenarios via Halo Shapes," arXiv:0912.5425 [hep-ph].

[117] A. Arvanitaki et al., "Astrophysical Probes of Unification," Phys. Rev. D79 (2009) 105022 arXiv:0812.2075 [hep-ph].

[118] A. Birkedal, K. Matchev, and M. Perelstein, "Dark matter at colliders: A model-independent approach," Phys. Rev. D70 (2004) 077701, arXiv:hep-ph/0403004

[119] J. L. Feng, S. Su, and F. Takayama, "Lower limit on dark matter production at the Large Hadron Collider," Phys. Rev. Lett. 96 (2006) 151802, arXiv: hep-ph/0503117.

[120] B. C. Allanach, G. Belanger, F. Boudjema, and A. Pukhov, "Requirements on collider data to match the precision of WMAP on supersymmetric dark matter," JHEP 12 (2004) 020, arXiv:hep-ph/0410091.

[121] T. Moroi, Y. Shimizu, and A. Yotsuyanagi, "Reconstructing dark matter density with e+ elinear collider in focus-point supersymmetry," Phys. Lett. B625 (2005) 79-87, arXiv:hep-ph/0505252.

[122] A. Birkedal et al., "Testing cosmology at the ILC," arXiv:hep-ph/0507214.

[123] E. A. Baltz, M. Battaglia, M. E. Peskin, and T. Wizansky, "Determination of dark matter properties at high-energy colliders," Phys. Rev. D74 (2006) 103521 arXiv:hep-ph/0602187.

[124] J. L. Feng, "ILC cosmology," arXiv:hep-ph/0509309.

[125] J. L. Feng, A. Rajaraman, and F. Takayama, "Superweakly-interacting massive particles," Phys. Rev. Lett. 91 (2003) 011302, arXiv:hep-ph/0302215.

[126] J. L. Feng, A. Rajaraman, and F. Takayama, "SuperWIMP dark matter signals from the early universe," Phys. Rev. D68 (2003) 063504, arXiv: hep-ph/0306024.

[127] L. M. Krauss, "New Constraints on Ino Masses from Cosmology. 1. Supersymmetric Inos," Nucl. Phys. B227 (1983) 556.

[128] D. V. Nanopoulos, K. A. Olive, and M. Srednicki, "After Primordial Inflation," Phys. Lett. B127 (1983) 30.

[129] M. Y. Khlopov and A. D. Linde, "Is It Easy to Save the Gravitino?," Phys. Lett. B138 (1984) 265-268.

[130] J. R. Ellis, J. E. Kim, and D. V. Nanopoulos, "Cosmological Gravitino Regeneration and Decay," Phys. Lett. B145 (1984) 181.

[131] R. Juszkiewicz, J. Silk, and A. Stebbins, "Constraints on Cosmologically Regenerated Gravitinos," Phys. Lett. B158 (1985) 463-467.

[132] M. Bolz, A. Brandenburg, and W. Buchmuller, "Thermal Production of Gravitinos," Nucl. Phys. B606 (2001) 518-544, arXiv:hep-ph/0012052.

[133] J. R. Ellis, K. A. Olive, Y. Santoso, and V. C. Spanos, "Gravitino dark matter in the CMSSM," Phys. Lett. B588 (2004) 7-16, arXiv:hep-ph/0312262.

[134] W. Buchmuller, K. Hamaguchi, M. Ratz, and T. Yanagida, "Supergravity at colliders," Phys. Lett. B588 (2004) 90-98, arXiv: hep-ph/0402179.

[135] F. Wang and J. M. Yang, "SuperWIMP dark matter scenario in light of WMAP," Eur. Phys. J. C38 (2004) 129-133, arXiv:hep-ph/0405186.

[136] L. Roszkowski, R. Ruiz de Austri, and K.-Y. Choi, "Gravitino dark matter in the CMSSM and implications for leptogenesis and the LHC," JHEP 08 (2005) 080, arXiv:hep-ph/0408227.

[137] K. Rajagopal, M. S. Turner, and F. Wilczek, "Cosmological implications of axinos," Nucl. 
Phys. B358 (1991) 447-470.

[138] L. Covi, J. E. Kim, and L. Roszkowski, "Axinos as cold dark matter," Phys. Rev. Lett. 82 (1999) 4180-4183, arXiv:hep-ph/9905212.

[139] L. Covi, H.-B. Kim, J. E. Kim, and L. Roszkowski, "Axinos as dark matter," JHEP 05 (2001) 033, arXiv:hep-ph/0101009.

[140] H. Baer and A. D. Box, "Fine-tuning favors mixed axion/axino cold dark matter over neutralinos in the minimal supergravity model," arXiv:0910.0333 [hep-ph].

[141] J. L. Feng, A. Rajaraman, and F. Takayama, "Graviton cosmology in universal extra dimensions," Phys. Rev. D68 (2003) 085018, arXiv:hep-ph/0307375.

[142] J. A. R. Cembranos, J. L. Feng, and L. E. Strigari, "Exotic collider signals from the complete phase diagram of minimal universal extra dimensions," Phys. Rev. D75 (2007) 036004, arXiv:hep-ph/0612157.

[143] X.-J. Bi, M.-z. Li, and X.-m. Zhang, "Quintessino as dark matter," Phys. Rev. D69 (2004) 123521, arXiv:hep-ph/0308218.

[144] R. Kitano and I. Low, "Grand unification, dark matter, baryon asymmetry, and the small scale structure of the universe," arXiv:hep-ph/0503112.

[145] L. E. Strigari, M. Kaplinghat, and J. S. Bullock, "Dark Matter Halos with Cores from Hierarchical Structure Formation," Phys. Rev. D75 (2007) 061303, arXiv:astro-ph/0606281.

[146] J. A. R. Cembranos and L. E. Strigari, "Diffuse MeV Gamma-rays and Galactic 511 keV Line from Decaying WIMP Dark Matter," Phys. Rev. D77 (2008) 123519, arXiv:0801.0630 [astro-ph].

[147] I. Albuquerque, G. Burdman, and Z. Chacko, "Neutrino telescopes as a direct probe of supersymmetry breaking," Phys. Rev. Lett. 92 (2004) 221802, arXiv:hep-ph/0312197.

[148] X.-J. Bi, J.-X. Wang, C. Zhang, and X.-m. Zhang, "Phenomenology of quintessino dark matter," Phys. Rev. D70 (2004) 123512, arXiv: hep-ph/0404263.

[149] Y. Huang, M. H. Reno, I. Sarcevic, and J. Uscinski, "Weak interactions of supersymmetric staus at high energies," Phys. Rev. D74 (2006) 115009, arXiv:hep-ph/0607216.

[150] M. Drees and X. Tata, "Signals for heavy exotics at hadron colliders and supercolliders," Phys. Lett. B252 (1990) 695-702.

[151] A. Rajaraman and B. T. Smith, "Discovering SUSY with $m_{0}^{2}<0$ in the first CERN LHC physics run," Phys. Rev. D75 (2007) 115015, arXiv:hep-ph/0612235.

[152] J. L. Feng and B. T. Smith, "Slepton trapping at the Large Hadron and International Linear Colliders," Phys. Rev. D71 (2005) 015004, arXiv:hep-ph/0409278.

[153] K. Hamaguchi, Y. Kuno, T. Nakaya, and M. M. Nojiri, "A study of late decaying charged particles at future colliders," Phys. Rev. D70 (2004) 115007, arXiv:hep-ph/0409248.

[154] A. De Roeck et al., "Supersymmetric benchmarks with non-universal scalar masses or gravitino dark matter," Eur. Phys. J. C49 (2007) 1041-1066, arXiv:hep-ph/0508198.

[155] A. Brandenburg, L. Covi, K. Hamaguchi, L. Roszkowski, and F. D. Steffen, "Signatures of axinos and gravitinos at colliders," Phys. Lett. B617 (2005) 99-111, arXiv:hep-ph/0501287.

[156] J. L. Feng, A. Rajaraman, and F. Takayama, "Probing gravitational interactions of elementary particles," Int. J. Mod. Phys. D13 (2004) 2355-2359, arXiv:hep-th/0405248.

[157] W. Hu and J. Silk, "Thermalization constraints and spectral distortions for massive unstable relic particles," Phys. Rev. Lett. 70 (1993) 2661-2664.

[158] R. Lamon and R. Durrer, "Constraining gravitino dark matter with the cosmic microwave 
background," Phys. Rev. D73 (2006) 023507, arXiv: hep-ph/0506229.

[159] D. J. Fixsen et al., "The Cosmic Microwave Background Spectrum from the Full COBE/FIRAS Data Set," Astrophys. J. 473 (1996) 576, arXiv:astro-ph/9605054.

[160] J. L. Feng, S. Su, and F. Takayama, "SuperWIMP gravitino dark matter from slepton and sneutrino decays," Phys. Rev. D70 (2004) 063514, arXiv: hep-ph/0404198.

[161] J. L. Feng, S. Su, and F. Takayama, "Supergravity with a gravitino LSP," Phys. Rev. D70 (2004) 075019, arXiv:hep-ph/0404231.

[162] M. Pospelov, "Particle physics catalysis of thermal big bang nucleosynthesis," Phys. Rev. Lett. 98 (2007) 231301, arXiv:hep-ph/0605215.

[163] K. Kohri and F. Takayama, "Big Bang Nucleosynthesis with Long Lived Charged Massive Particles," Phys. Rev. D76 (2007) 063507, arXiv:hep-ph/0605243.

[164] M. Kaplinghat and A. Rajaraman, "Big Bang Nucleosynthesis with Bound States of Long-lived Charged Particles," Phys. Rev. D74 (2006) 103004, arXiv:astro-ph/0606209.

[165] M. Kawasaki, K. Kohri, and T. Moroi, "Big-Bang Nucleosynthesis with Long-Lived Charged Slepton," Phys. Lett. B649 (2007) 436-439, arXiv:hep-ph/0703122.

[166] F. Takayama, "Extremely Long-Lived Charged Massive Particles as A Probe for Reheating of the Universe," Phys. Rev. D77 (2008) 116003, arXiv:0704.2785 [hep-ph].

[167] M. Ratz, K. Schmidt-Hoberg, and M. W. Winkler, "A note on the primordial abundance of stau NLSPs," JCAP 0810 (2008) 026, arXiv:0808.0829 [hep-ph].

[168] T. Jittoh et al., "Possible solution to the ${ }^{7} \mathrm{Li}$ problem by the long lived stau," Phys. Rev. D76 (2007) 125023, arXiv:0704.2914 [hep-ph].

[169] D. Cumberbatch et al., "Solving the cosmic lithium problems with primordial latedecaying particles," Phys. Rev. D76 (2007) 123005, arXiv:0708.0095 [astro-ph].

[170] S. Bailly, K. Jedamzik, and G. Moultaka, "Gravitino Dark Matter and the Cosmic Lithium Abundances," Phys. Rev. D80 (2009) 063509, arXiv:0812.0788 [hep-ph].

[171] K. Jedamzik and M. Pospelov, "Big Bang Nucleosynthesis and Particle Dark Matter," New J. Phys. 11 (2009) 105028, arXiv:0906.2087 [hep-ph].

[172] S. Bailly, K.-Y. Choi, K. Jedamzik, and L. Roszkowski, "A Re-analysis of Gravitino Dark Matter in the Constrained MSSM," JHEP 05 (2009) 103, arXiv:0903.3974 [hep-ph].

[173] W. B. Lin, D. H. Huang, X. Zhang, and R. H. Brandenberger, "Non-thermal production of WIMPs and the sub-galactic structure of the universe," Phys. Rev. Lett. 86 (2001) 954, arXiv:astro-ph/0009003.

[174] K. Sigurdson and M. Kamionkowski, "Charged-particle decay and suppression of small-scale power," Phys. Rev. Lett. 92 (2004) 171302, arXiv:astro-ph/0311486.

[175] S. Profumo, K. Sigurdson, P. Ullio, and M. Kamionkowski, "A Running spectral index in supersymmetric dark-matter models with quasi-stable charged particles," Phys. Rev. D71 (2005) 023518, arXiv:astro-ph/0410714.

[176] M. Kaplinghat, "Dark matter from early decays," Phys. Rev. D72 (2005) 063510, arXiv: astro-ph/0507300.

[177] J. A. R. Cembranos, J. L. Feng, A. Rajaraman, and F. Takayama, "SuperWIMP solutions to small scale structure problems," Phys. Rev. Lett. 95 (2005) 181301, arXiv:hep-ph/0507150.

[178] K. Jedamzik, M. Lemoine, and G. Moultaka, "Gravitino, Axino, Kaluza-Klein Graviton Warm and Mixed Dark Matter and Reionisation," JCAP 0607 (2006) 010, arXiv:astro-ph/0508141.

[179] F. Borzumati, T. Bringmann, and P. Ullio, "Dark matter from late decays and the 
small-scale structure problems," Phys. Rev. D77 (2008) 063514, arXiv:hep-ph/0701007.

[180] J. R. Primack, "Cosmology: small scale issues revisited," arXiv:0909.2247 [astro-ph.CO].

[181] M. Dine, W. Fischler, and M. Srednicki, "Supersymmetric Technicolor," Nucl. Phys. B189 (1981) 575-593.

[182] S. Dimopoulos and S. Raby, "Supercolor," Nucl. Phys. B192 (1981) 353.

[183] C. R. Nappi and B. A. Ovrut, "Supersymmetric Extension of the SU(3) x SU(2) x U(1) Model," Phys. Lett. B113 (1982) 175.

[184] L. Alvarez-Gaume, M. Claudson, and M. B. Wise, "Low-Energy Supersymmetry," Nucl. Phys. B207 (1982) 96.

[185] M. Dine, A. E. Nelson, and Y. Shirman, "Low-energy dynamical supersymmetry breaking simplified," Phys. Rev. D51 (1995) 1362-1370, arXiv:hep-ph/9408384

[186] M. Dine, A. E. Nelson, Y. Nir, and Y. Shirman, "New tools for low-energy dynamical supersymmetry breaking," Phys. Rev. D53 (1996) 2658-2669, arXiv:hep-ph/9507378.

[187] H. Pagels and J. R. Primack, "Supersymmetry, Cosmology and New TeV Physics," Phys. Rev. Lett. 48 (1982) 223.

[188] U. Seljak, A. Makarov, P. McDonald, and H. Trac, "Can sterile neutrinos be the dark matter?," Phys. Rev. Lett. 97 (2006) 191303, arXiv:astro-ph/0602430.

[189] M. Viel, J. Lesgourgues, M. G. Haehnelt, S. Matarrese, and A. Riotto, "Can sterile neutrinos be ruled out as warm dark matter candidates?," Phys. Rev. Lett. 97 (2006) 071301, arXiv: astro-ph/0605706.

[190] E. A. Baltz and H. Murayama, "Gravitino warm dark matter with entropy production," JHEP 05 (2003) 067, arXiv:astro-ph/0108172.

[191] M. Viel, J. Lesgourgues, M. G. Haehnelt, S. Matarrese, and A. Riotto, "Constraining warm dark matter candidates including sterile neutrinos and light gravitinos with WMAP and the Lyman- alpha forest," Phys. Rev. D71 (2005) 063534, arXiv:astro-ph/0501562.

[192] CDF Collaboration, T. Aaltonen et al., "Search for Supersymmetry with Gauge-Mediated Breaking in Diphoton Events with Missing Transverse Energy at CDF II," Phys. Rev. Lett. 104 (2010) 011801, arXiv:0910.3606 [hep-ex].

[193] I. Y. Kobsarev, L. B. Okun, and I. Y. Pomeranchuk Sov. J. Nucl. Phys. 3 (1966) 837.

[194] S. I. Blinnikov and M. Y. Khlopov, "On possible effects of 'mirror' particles," Sov. J. Nucl. Phys. 36 (1982) 472.

[195] R. Foot, H. Lew, and R. R. Volkas, "A Model with fundamental improper space-time symmetries," Phys. Lett. B272 (1991) 67-70.

[196] H. M. Hodges, "Mirror baryons as the dark matter," Phys. Rev. D47 (1993) 456-459.

[197] Z. G. Berezhiani, A. D. Dolgov, and R. N. Mohapatra, "Asymmetric Inflationary Reheating and the Nature of Mirror Universe," Phys. Lett. B375 (1996) 26-36, arXiv:hep-ph/9511221.

[198] R. H. Cyburt, B. D. Fields, K. A. Olive, and E. Skillman, "New BBN limits on Physics Beyond the Standard Model from He4," Astropart. Phys. 23 (2005) 313-323, arXiv:astro-ph/0408033.

[199] B. Fields and S. Sarkar, "Big-bang nucleosynthesis (PDG mini-review)," arXiv:astro-ph/0601514.

[200] P. Ciarcelluti and A. Lepidi, "Thermodynamics of the early Universe with mirror dark matter," Phys. Rev. D78 (2008) 123003, arXiv:0809.0677 [astro-ph].

[201] E. W. Kolb, D. Seckel, and M. S. Turner, "The Shadow World," Nature 314 (1985) 


\section{5-419.}

[202] J. L. Feng and J. Kumar, "The WIMPless Miracle: Dark-Matter Particles without WeakScale Masses or Weak Interactions," Phys. Rev. Lett. 101 (2008) 231301, arXiv:0803.4196 [hep-ph].

[203] D. Hooper and K. M. Zurek, "Natural supersymmetric model with MeV dark matter," Phys. Rev. D77 (2008) 087302, arXiv:0801.3686 [hep-ph].

[204] J. L. Feng, H. Tu, and H.-B. Yu, "Thermal Relics in Hidden Sectors," JCAP 0810 (2008) 043, arXiv:0808.2318 [hep-ph].

[205] R. Foot, "Mirror dark matter and the new DAMA/LIBRA results: A simple explanation for a beautiful experiment," Phys. Rev. D78 (2008) 043529, arXiv:0804.4518 [hep-ph].

[206] Y. G. Kim and S. Shin, "Singlet Fermionic Dark Matter explains DAMA signal," JHEP 05 (2009) 036, arXiv:0901.2609 [hep-ph].

[207] A. Bottino, F. Donato, N. Fornengo, and S. Scopel, "Lower bound on the neutralino mass from new data on CMB and implications for relic neutralinos," Phys. Rev. D68 (2003) 043506, arXiv: hep-ph/0304080.

[208] F. Petriello and K. M. Zurek, "DAMA and WIMP dark matter," JHEP 09 (2008) 047, arXiv:0806.3989 [hep-ph].

[209] G. Angloher et al., "Limits on WIMP dark matter using sapphire cryogenic detectors," Astropart. Phys. 18 (2002) 43-55.

[210] CDMS Collaboration, D. S. Akerib et al., "Limits on spin-independent WIMP nucleon interactions from the two-tower run of the Cryogenic Dark Matter Search," Phys. Rev. Lett. 96 (2006) 011302, arXiv:astro-ph/0509259.

[211] TEXONO Collaboration, S. T. Lin et al., "New limits on spin-independent and spin-dependent couplings of low-mass WIMP dark matter with a germanium detector at a threshold of 220 eV," Phys. Rev. D79 (2009) 061101, arXiv:0712.1645 [hep-ex].

[212] CoGeNT Collaboration, C. E. Aalseth et al., "Experimental constraints on a dark matter origin for the DAMA annual modulation effect," Phys. Rev. Lett. 101 (2008) 251301, arXiv:0807.0879 [astro-ph]

[213] G. D. Kribs, T. Plehn, M. Spannowsky, and T. M. P. Tait, "Four generations and Higgs physics," Phys. Rev. D76 (2007) 075016, arXiv:0706.3718 [hep-ph].

[214] J. Alwall, J. L. Feng, J. Kumar, and S. Su, "Dark Matter-Motivated Searches for Exotic 4th Generation Quarks in Tevatron and Early LHC Data," arXiv:1002.3366 [hep-ph].

[215] D. McKeen, "WIMPless Dark Matter and Meson Decays with Missing Energy," Phys. Rev. D79 (2009) 114001, arXiv:0903.4982 [hep-ph].

[216] B. Holdom, "Two U(1)'s and Epsilon Charge Shifts," Phys. Lett. B166 (1986) 196.

[217] S. Davidson, S. Hannestad, and G. Raffelt, "Updated bounds on milli-charged particles," JHEP 05 (2000) 003, arXiv:hep-ph/0001179.

[218] M. L. Perl, E. R. Lee, and D. Loomba, "A brief review of the search for isolatable fractional charge elementary particles," Mod. Phys. Lett. A19 (2004) 2595-2610.

[219] D. N. Spergel and P. J. Steinhardt, "Observational evidence for self-interacting cold dark matter," Phys. Rev. Lett. 84 (2000) 3760-3763, arXiv:astro-ph/9909386.

[220] J. L. Feng, M. Kaplinghat, H. Tu, and H.-B. Yu, "Hidden Charged Dark Matter," JCAP 0907 (2009) 004, arXiv:0905.3039 [hep-ph].

[221] L. Ackerman, M. R. Buckley, S. M. Carroll, and M. Kamionkowski, "Dark Matter and Dark Radiation," Phys. Rev. D79 (2009) 023519, arXiv:0810.5126 [hep-ph].

[222] R. Dave, D. N. Spergel, P. J. Steinhardt, and B. D. Wandelt, "Halo Properties in 
Cosmological Simulations of Self- Interacting Cold Dark Matter," Astrophys. J. 547 (2001) 574-589, arXiv:astro-ph/0006218.

[223] N. Yoshida, V. Springel, S. D. M. White, and G. Tormen, "Collisional dark matter and the structure of dark halos," Astrophys. J. 535 (2000) L103, arXiv:astro-ph/0002362.

[224] J. Miralda-Escude, "A Test of the Collisional Dark Matter Hypothesis from Cluster Lensing," arXiv:astro-ph/0002050.

[225] D. A. Buote, T. E. Jeltema, C. R. Canizares, and G. P. Garmire, "Chandra Evidence for a Flattened, Triaxial Dark Matter Halo in the Elliptical Galaxy NGC 720," Astrophys. J. 577 (2002) 183-196, arXiv:astro-ph/0205469.

[226] P. J. Humphrey et al., "A Chandra View of Dark Matter in Early-Type Galaxies," Astrophys. J. 646 (2006) 899-918, arXiv:astro-ph/0601301.

[227] S. Gardner, "Shedding Light on the Symmetries of Dark Matter," arXiv:1001.2176 [hep-ph].

[228] S. Dodelson and L. M. Widrow, "Sterile Neutrinos as Dark Matter," Phys. Rev. Lett. 72 (1994) 17-20, arXiv: hep-ph/9303287.

[229] K. Abazajian and S. M. Koushiappas, "Constraints on sterile neutrino dark matter," Phys. Rev. D74 (2006) 023527, arXiv:astro-ph/0605271.

[230] X.-D. Shi and G. M. Fuller, "A new dark matter candidate: Non-thermal sterile neutrinos," Phys. Rev. Lett. 82 (1999) 2832-2835, arXiv:astro-ph/9810076.

[231] T. Asaka, M. Laine, and M. Shaposhnikov, "Lightest sterile neutrino abundance within the nuMSM," JHEP 01 (2007) 091, arXiv:hep-ph/0612182.

[232] M. Laine and M. Shaposhnikov, "Sterile neutrino dark matter as a consequence of nuMSMinduced lepton asymmetry," JCAP 0806 (2008) 031, arXiv:0804.4543 [hep-ph].

[233] M. Shaposhnikov and I. Tkachev, "The nuMSM, inflation, and dark matter," Phys. Lett. B639 (2006) 414-417, arXiv: hep-ph/0604236.

[234] A. Kusenko, "Sterile neutrinos, dark matter, and the pulsar velocities in models with a Higgs singlet," Phys. Rev. Lett. 97 (2006) 241301, arXiv:hep-ph/0609081.

[235] K. Petraki and A. Kusenko, "Dark-matter sterile neutrinos in models with a gauge singlet in the Higgs sector," Phys. Rev. D77 (2008) 065014, arXiv:0711.4646 [hep-ph].

[236] P. B. Pal and L. Wolfenstein, "Radiative Decays of Massive Neutrinos," Phys. Rev. D25 (1982) 766.

[237] K. N. Abazajian, "Detection of Dark Matter Decay in the X-ray," arXiv:0903.2040 [astro-ph.CO].

[238] M. Loewenstein and A. Kusenko, "Dark Matter Search Using Chandra Observations of Willman 1, and a Spectral Feature Consistent with a Decay Line of a $5 \mathrm{keV}$ Sterile Neutrino," arXiv:0912.0552 [astro-ph.HE].

[239] A. Boyarsky et al., "Searching for dark matter in X-rays: how to check the dark matter origin of a spectral feature," arXiv:1001.0644 [astro-ph.C0].

[240] A. Kusenko and M. Loewenstein, "Searching for dark matter in X-rays: how not to check the dark matter origin of a spectral feature," arXiv:1001.4055 [astro-ph.CO].

[241] A. Kusenko, "Sterile neutrinos: the dark side of the light fermions," Phys. Rept. 481 (2009) 1-28, arXiv:0906.2968 [hep-ph].

[242] A. Boyarsky, J. Lesgourgues, O. Ruchayskiy, and M. Viel, "Lyman-alpha constraints on warm and on warm-plus-cold dark matter models," JCAP 0905 (2009) 012, arXiv:0812.0010 [astro-ph]

[243] A. Boyarsky, O. Ruchayskiy, and M. Shaposhnikov, "The role of sterile neutrinos in 
cosmology and astrophysics," Ann. Rev. Nucl. Part. Sci. 59 (2009) 191-214, arXiv:0901.0011 [hep-ph].

[244] R. D. Peccei and H. R. Quinn, "CP Conservation in the Presence of Instantons," Phys. Rev. Lett. 38 (1977) 1440-1443.

[245] F. Wilczek, "Problem of Strong p and t Invariance in the Presence of Instantons," Phys. Rev. Lett. 40 (1978) 279-282.

[246] S. Weinberg, "A New Light Boson?," Phys. Rev. Lett. 40 (1978) 223-226.

[247] J. Preskill, M. B. Wise, and F. Wilczek, "Cosmology of the invisible axion," Phys. Lett. B120 (1983) 127-132.

[248] L. F. Abbott and P. Sikivie, "A cosmological bound on the invisible axion," Phys. Lett. B120 (1983) 133-136.

[249] M. Dine and W. Fischler, "The not-so-harmless axion," Phys. Lett. B120 (1983) 137-141.

[250] S. J. Asztalos, L. J. Rosenberg, K. van Bibber, P. Sikivie, and K. Zioutas, "Searches for astrophysical and cosmological axions," Ann. Rev. Nucl. Part. Sci. 56 (2006) 293-326.

[251] J. E. Kim, "Weak Interaction Singlet and Strong CP Invariance," Phys. Rev. Lett. 43 (1979) 103.

[252] M. A. Shifman, A. I. Vainshtein, and V. I. Zakharov, "Can Confinement Ensure Natural CP Invariance of Strong Interactions?," Nucl. Phys. B166 (1980) 493.

[253] M. Dine, W. Fischler, and M. Srednicki, "A Simple Solution to the Strong CP Problem with a Harmless Axion," Phys. Lett. B104 (1981) 199.

[254] A. R. Zhitnitsky, "On Possible Suppression of the Axion Hadron Interactions. (In Russian)," Sov. J. Nucl. Phys. 31 (1980) 260.

[255] G. G. Raffelt, "Astrophysical axion bounds," Lect. Notes Phys. 741 (2008) 51-71, arXiv:hep-ph/0611350.

[256] K. J. Bae, J.-H. Huh, and J. E. Kim, "Update of axion CDM energy," JCAP 0809 (2008) 005, arXiv:0806.0497 [hep-ph].

[257] S. Chang, C. Hagmann, and P. Sikivie, "Studies of the motion and decay of axion walls bounded by strings," Phys. Rev. D59 (1999) 023505, arXiv:hep-ph/9807374.

[258] C. Hagmann, S. Chang, and P. Sikivie, "Axion radiation from strings," Phys. Rev. D63 (2001) 125018, arXiv: hep-ph/0012361.

[259] R. A. Battye and E. P. S. Shellard, "Axion string constraints," Phys. Rev. Lett. 73 (1994) 2954-2957, arXiv:astro-ph/9403018.

[260] M. Yamaguchi, M. Kawasaki, and J. Yokoyama, "Evolution of axionic strings and spectrum of axions radiated from them," Phys. Rev. Lett. 82 (1999) 4578-4581,

arXiv:hep-ph/9811311.

[261] A. D. Linde, "Inflation and Axion Cosmology," Phys. Lett. B201 (1988) 437.

[262] M. Tegmark, A. Aguirre, M. Rees, and F. Wilczek, "Dimensionless constants, cosmology and other dark matters," Phys. Rev. D73 (2006) 023505, arXiv:astro-ph/0511774.

[263] P. Sikivie, "Experimental tests of the invisible axion," Phys. Rev. Lett. 51 (1983) 1415. 